\title{
A WORKSITE EXAMINATION OF \\ THE TRANSTHEORETICAL MODEL IN EXERCISE
}

\section{DISSERTATION}

\begin{abstract}
Presented in Partial Fulfillment of the Requirements
for the Degree Doctor of Philosophy in the Graduate
\end{abstract}

School of the Ohio State University

By

Christopher L. Fink, M.S.

The Ohio State University

2007

Dissertation Committee:

Professor Janet Buckworth, Advisor

Approved by

Professor Rick Petosa

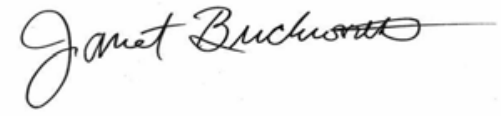

Professor Brian Focht

Advisor

Education Graduate Program 



\begin{abstract}
The purpose of this study was to test the ability of 3-month and 6-month changes in the components of the transtheoretical model (TTM - stages of change, processes of change, self-efficacy, decisional balance) to predict 6-month inactive to active stage transition in a randomly selected worksite sample $(N=60)$. Participants completed a webbased survey at baseline, 3 months, and 6 months.

The results indicated that changes in behavioral processes of change from baseline to 3 months, changes in self-efficacy and behavioral processes of change from 3 to 6 months, and self-efficacy, cognitive processes of change, and decisional balancecons were significant predictors $(\mathrm{p}<.05)$ of 6 -month stage transition. The findings cast some doubt on the rigidity of the 6-month component in the TTM, as well as the idea that behavioral processes of change are most important in the change process during the later stages.
\end{abstract}




\section{ACKNOWLEDGMENTS}

I would like to thank my advisor, Dr. Janet Buckworth, for her patience and guidance throughout the processes of designing, developing, and reporting this research. Her enthusiasm for this area of inquiry became mine, and was manifested in this project.

I thank my committee members; Dr. Rick Petosa and Dr. Brian Focht for their helpful and insightful suggestions that helped make this project a success.

I thank Dr. Amy Ferketich for her assistance in the design and analysis of this project.

I thank Dr. Brian Hortz and Dr. Emily Stevens for their input on design and statistical analysis. Their assistance was invaluable.

I thank Chuck Shiebler and Dr. Jackie Goodway-Shiebler for affording me the opportunities I needed to succeed.

I thank Dr. Nancy Knop and Margie Shade for their belief in my abilities.

I also thank my former advisors and mentors, Dr. Jack Raglin, Dr. R. Lee Gardiner, and Joseph Susi for building my knowledge and interest.

I thank Dona Fink (my mother), Thomas Fink (my father), and Linda Fink (my step-mother) for their support throughout my educational journey. Thank you also to Mark Samples for standing with me through it all.

Finally, I'd like to thank my wife, Julie, for her assistance, patience, support, and love throughout this process. 
VITA

March 13, 1972.............. Born - Parma, Ohio

1999......................... . in Exercise Science, Lake Superior State University

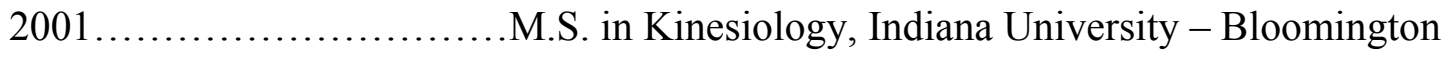

2001-2005.................. Graduate Teaching / Administrative Assistant

The Ohio State University

2006, 2007................Instructor, The Ohio State University

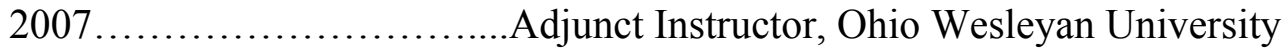

\section{PUBLICATIONS}

Research Publication

1. Buckworth, J., Fink, C., Rozorea, M., Jansen, J., \& Mattern, C. (2004). Ratings of perceived exertion are not influenced by exercise stage of change in physically active college students. Perceptual and Motor Skills, 99(3), 871-882.

\section{FIELDS OF STUDY}

Major Field: Education 


\section{TABLE OF CONTENTS}

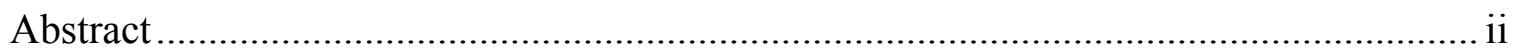

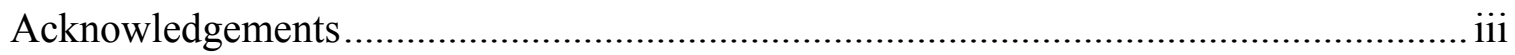

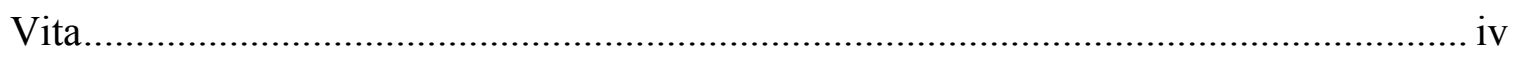

List of Tables ................................................................................................... viii

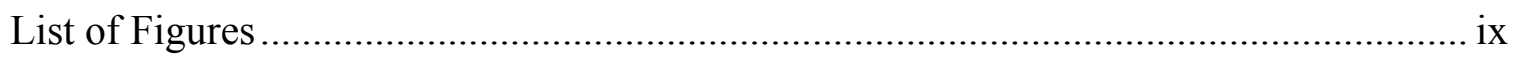

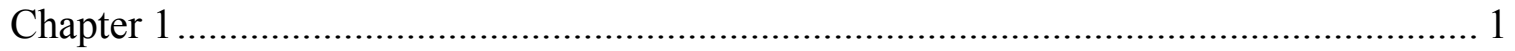

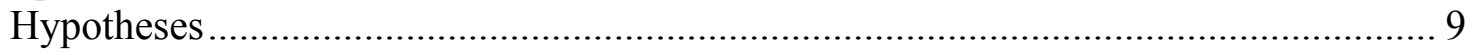

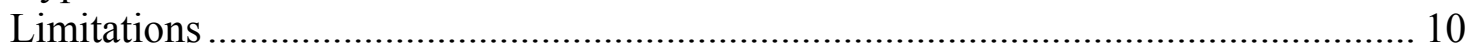

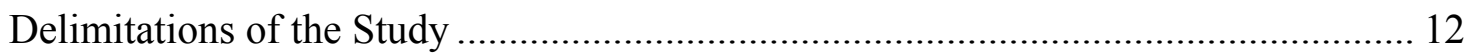

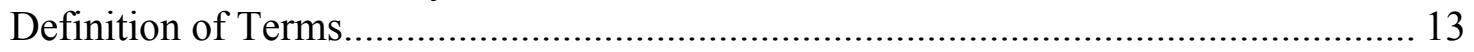

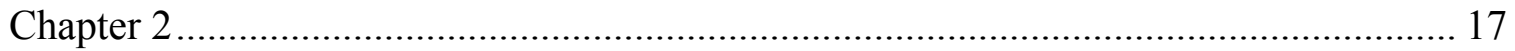

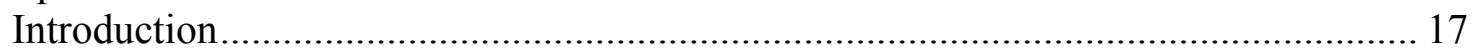

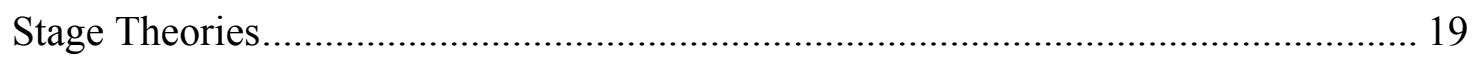

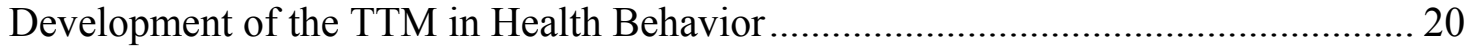

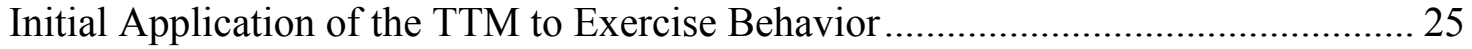

Staging in Physical Activity and Exercise - Role of Behavioral Definition ............... 28

Cross-Sectional Examinations ............................................................................. 36

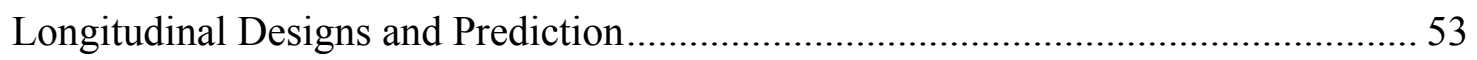

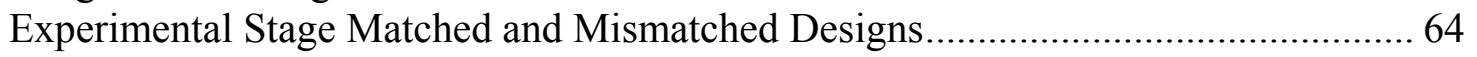

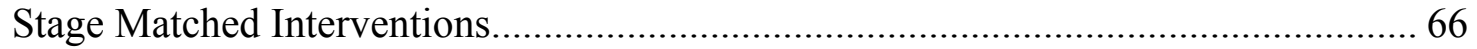

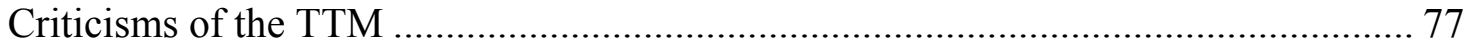

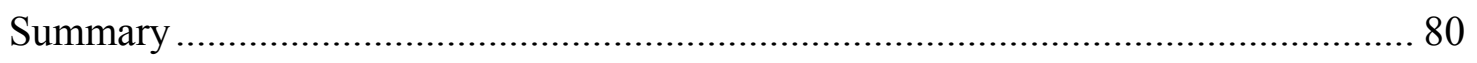

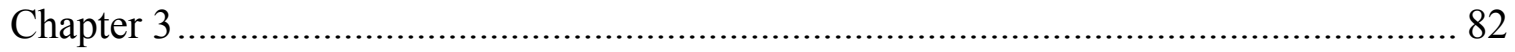

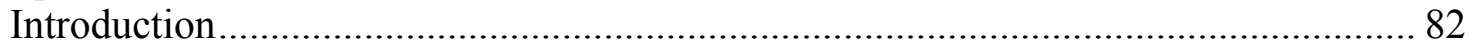

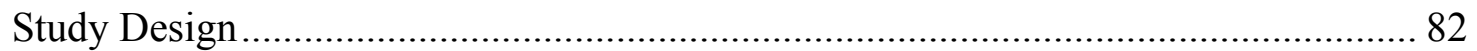

Inclusion Criteria .......................................................................................... 85

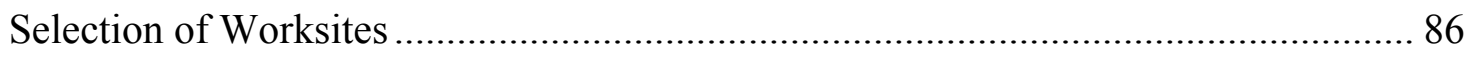

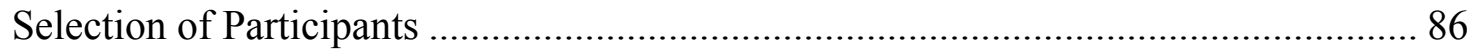

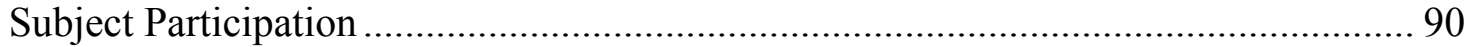

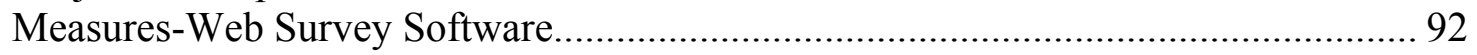

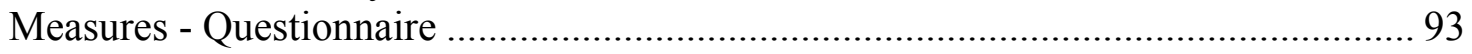




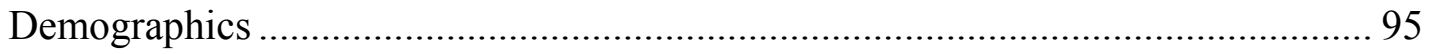

Check for Injury or Illness ................................................................................. 96

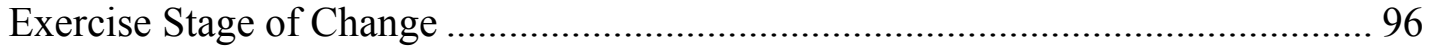

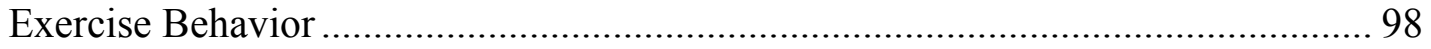

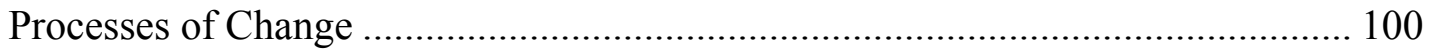

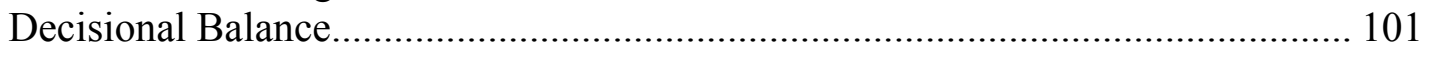

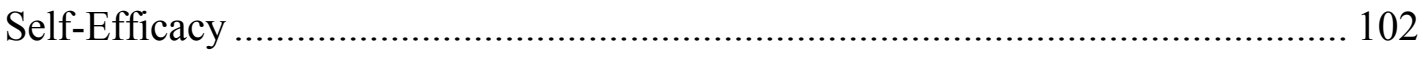

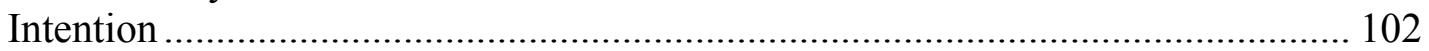

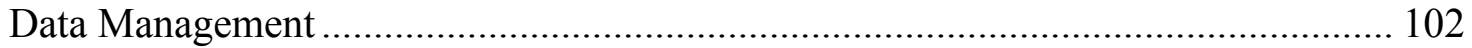

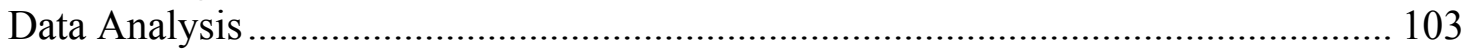

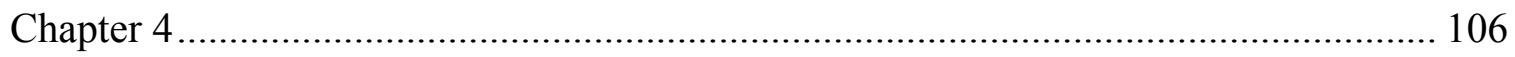

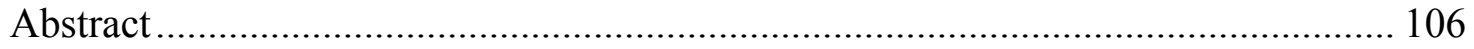

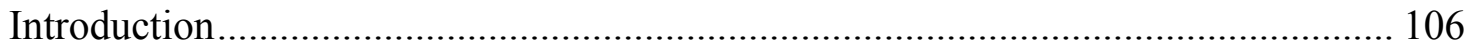

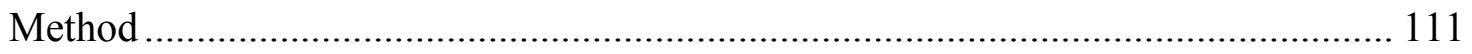

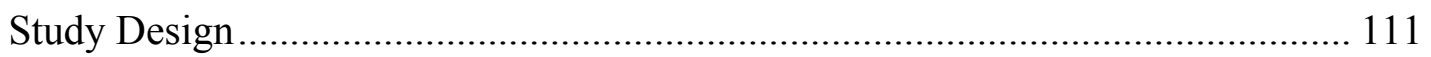

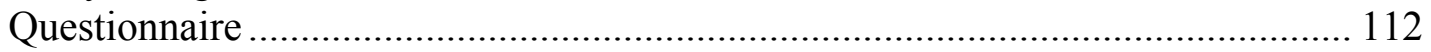

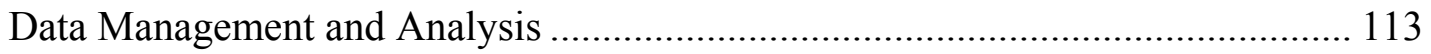

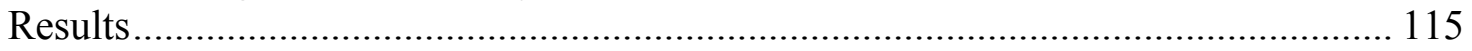

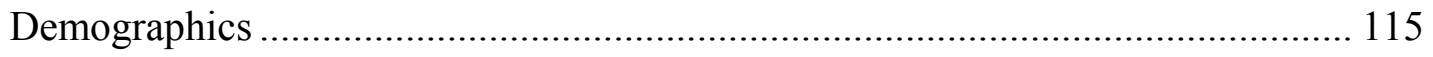

Logistic Regression.................................................................................... 117

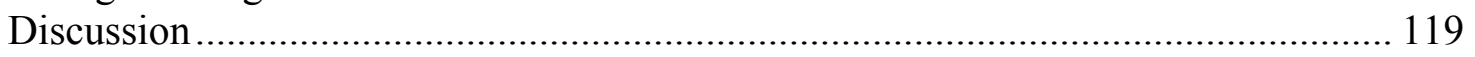

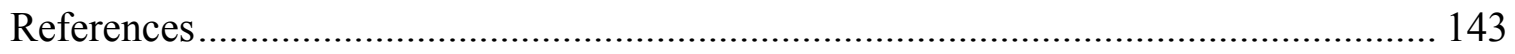

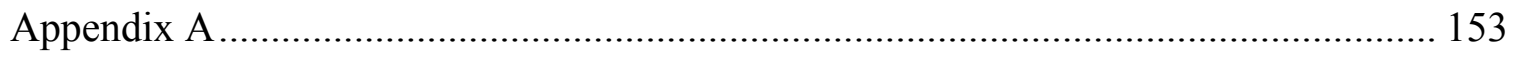

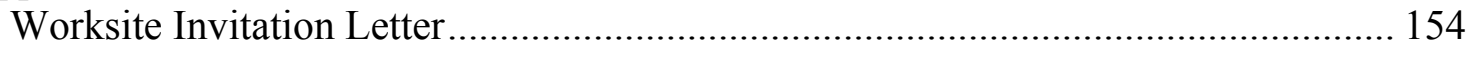

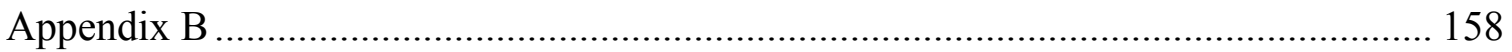

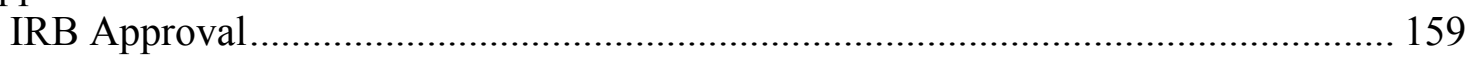

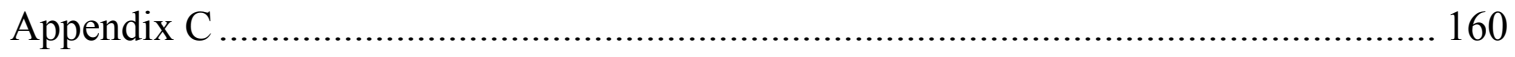

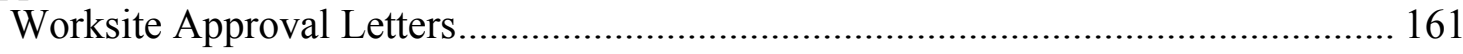

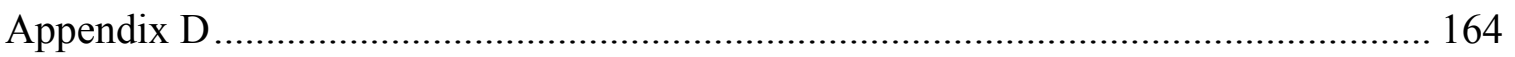

Participant Invitation and Follow-Up Letters ......................................................... 165

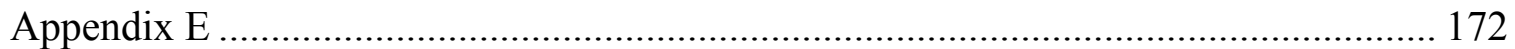

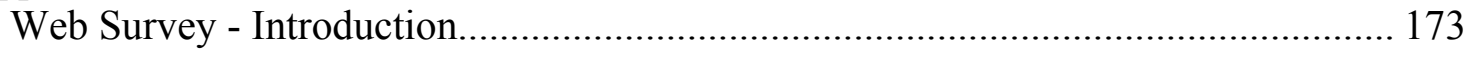




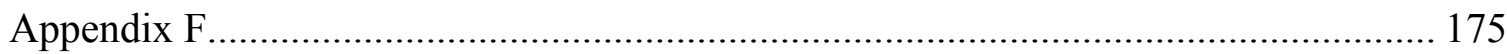

Web Survey - Demographics.............................................................................. 176

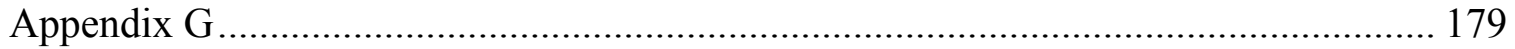

Web Survey - Check for Illness and Injury ............................................................ 180

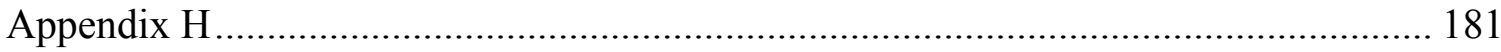

Web Survey - Stage of Change 5 Choice..................................................................... 182

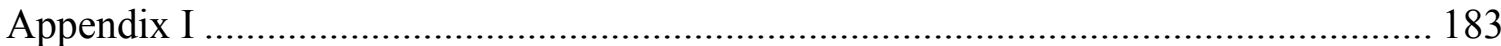

Web Survey - Exercise Recall - Moderate................................................................ 184

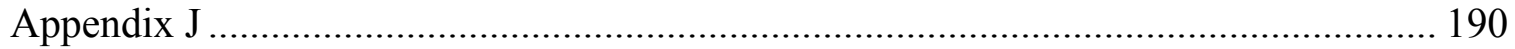

Web Survey - Exercise Recall - Vigorous ................................................................. 191

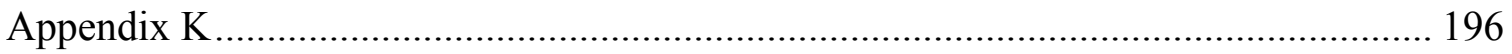

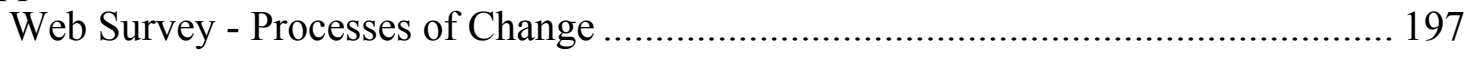

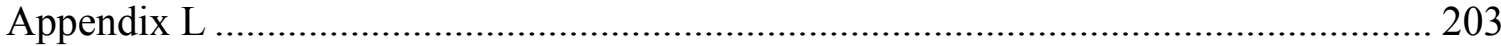

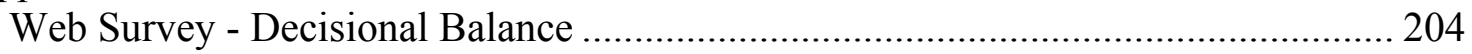

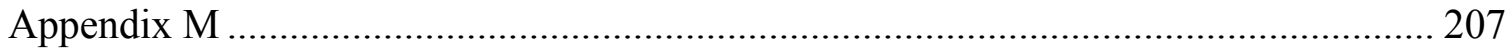

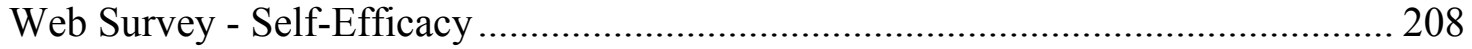

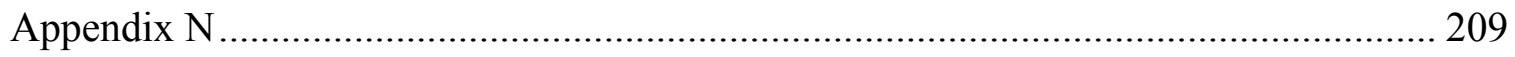

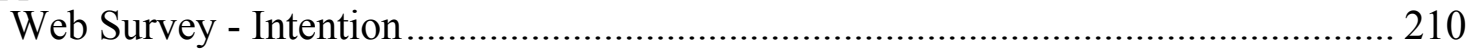

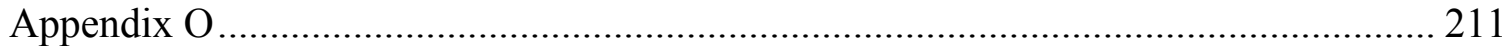

Web Survey - Stage of Change - True False........................................................... 212

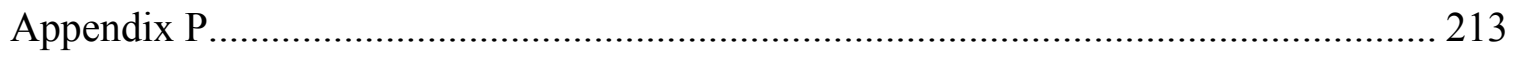

Web Survey - Thank You Page .......................................................................... 214 


\section{LIST OF TABLES}

Table

Page

Table 2.1 TTM-Based Exercise Intervention Studies................69

Table 3.1 Questionnaire Components at Baseline and Each

Follow-Up......................................... 85

Table 3.2 Initial Sampling Scheme............................ 89

Table 4.1 Demographic Characteristics of Target Sample..............129

Table 4.2 TTM Construct Means and Standard Deviations for S-I and I-A Participants................................130

Table 4.3 P-values, Effect Size, and Power for 2 x 3 (Group x Time) Mixed Model ANOVA on Each TTM Construct............. 131

Table 4.4 Mean and Standard Deviation of Total Weekly Minutes of Exercise for S-I and I-A Participants by Measurement Time................................................... 132

Table 4.5 Correlation Matrix of All TTM Constructs at Each Measurement Point. 


\section{LIST OF FIGURES}

Figure Page

Figure $3.1 \quad$ Likert Scale with Period at Left........................ 93

Figure $4.1 \quad$ Participant Flow Chart.............................. 134

Figure 4.2 Mean Self-Efficacy Scores for S-Iand I-A Groups by Measurement Time......................................135

Figure 4.3 Mean Cognitive Processes of Change Scores for S-I and I-A Groups by Measurement Time................. 136

Figure 4.4 Mean Behavioral Processes of Change Scores for S-I and I-A Groups by Measurement Time................... 137

Figure 4.5 Mean Decisional Balance-Pro Scores for S-I and I-A Groups by Measurement Time..........................................138

Figure 4.6 Mean Decisional Balance - Con Scores for S-I and I-A Groups by Measurement Time........................ 139

Figure 4.7 Mean Total Minutes of Exercise for S-I and I-A Groups by Measurement Time........................ 140

Figure 4.8 Odds Ratios for TTM Constructs Retained in Multivariate Logistic Regression Models by Time.

Figure 4.9 Mean Change Scores for TTM Constructs Showing Contribution of Measurement Times..................... 142 


\section{CHAPTER 1}

\section{INTRODUCTION}

\section{Background}

Physical inactivity has been well-documented as an independent risk factor for a variety of chronic diseases, including coronary heart disease (CHD), type II diabetes, hypertension, and colon cancer (U.S. Department of Health and Human Services, 2000). An inactive lifestyle has been shown to contribute to mental health issues, osteoporosis, and obesity (U.S. Department of Health and Human Services, 1996). Regular exercise is protective of these same chronic diseases, but, participation rates are disappointing, with

over $60 \%$ of U.S. adults engaging in insufficient levels for garnering these health benefits (Centers for Disease Control and Prevention, 2000).

Centers for Disease Control and Prevention (CDC) data show that physical inactivity is associated with needing more medication, visiting a physician more often, and being hospitalized more often (Centers for Disease Control and Prevention, 2000). Direct medical costs from physical inactivity have also risen from \$29 billion in 1987 to more than \$76 billion in 2000 (Centers for Disease Control and Prevention, 2000). Obesity, to which physical inactivity contributes, is estimated to cost $\$ 117$ billion annually (Centers for Disease Control and Prevention, 2000). Despite the efforts of 
health and exercise professionals, most exercisers drop out within six months (Dishman, 1988).

There are a variety of explanations for unsuccessful physical activity interventions. Several different behavioral theories (such as social cognitive theory and behavioral modification) have been used by researchers as guides to analyze these disappointing outcomes. Behavioral theories are also tools that are used in planning physical activity interventions. As such, theory can be used as a "road-map," directing the development of the intervention so that constructs (correlates) that are proposed to be related to the target behavior are considered when selecting intervention strategies.

Though the use of theory may appear on the surface to be an uncomplicated solution to designing effective interventions, the application is far more complex. In fact, interventions are often based on a premature use of theory, that is, constructs strongly correlated with the target outcome are assumed to have causal relationships even before such relationships have been verified through experimental studies or even demonstrated to track longitudinally (Dishman, 2001). In addition, theories developed for other health behaviors, such as smoking cessation and nutritional choices, have been adapted for use with exercise (Ajzen, 1991; Fishbein, 1967; Prochaska \& DiClemente, 1983). The application of these theories to exercise calls into question their validity, as the dynamics of physical activity are quite different from other health behaviors. For example, the typical objective for exercise interventions is adoption of the behavior, while the goal for smoking interventions is cessation. Other dynamics of the health behavior can also vary, such as frequency of engagement in the behavior of change and physiological 
characteristics (e.g., neurological responses to nicotine versus cardiovascular activation with exercise).

Baranowski, Anderson, and Carmack (1998) reviewed physical activity interventions and correlational studies and found that $30 \%$ or less of the variability in physical activity behavior was accounted for by theoretical constructs. In addition, most of the intervention studies did not actually measure the mediating variables, and so it is unclear whether the interventions were actually affecting the proposed mediating variables, or whether some confounding factor skewed the results (Baranowski et al., 1998).

Most behavioral models and theories (e.g. Social Cognitive Theory, Theory of Planned Behavior/Reasoned Action) have a series of variables that are proposed to move individuals from inactive to active, without consideration of the pre-existing differences in motivational readiness. These theories do not account for cognitions and behaviors necessary to move from sedentary to active that likely occur during intermediary steps, which may not involve changes in physical activity behavior.

The transtheoretical model of behavior change (TTM) (Prochaska \& DiClemente, 1983) has been used and studied extensively in physical activity behavior interventions and research (Marshall \& Biddle, 2001). As the name suggests, the TTM was developed from a comparative analysis of a variety of psychotherapy and behavior change models, as well as from a naturalistic inquiry into the smoking cessation process (Prochaska \& DiClemente, 1983; Prochaska, Redding, \& Evers, 2002). Several core constructs were developed, including six stages of change (Precontemplation, Contemplation, Preparation, Action, and Maintenance, and Termination), which are defined temporally 
both by the health behavior and an individual's motivational readiness to change, generally in six-month increments (Prochaska \& DiClemente, 1983; Prochaska et al., 2002). The Termination stage has not been thoroughly examined in the context of exercise, as it may be difficult to use based on the demands of the behavior. The Termination stage indicates the point at which the individual is no longer tempted to regress to the previous behavioral stage(s) and has 100\% self-efficacy for the behavior (Prochaska \& Velicer, 1997b). Three constructs are hypothesized to mediate the movement through the stages of change. First, 10 processes, or strategies that individuals use to move through the stages of change, were identified. They were separated into five cognitive or experiential and five behavioral processes of change. Additionally, decisional balance (i.e., weighing the pros and cons of behavior change) and self-efficacy were included in the model (Marshall \& Biddle, 2001; Prochaska et al., 2002). It was initially conceptualized that individuals moved through the stages in a linear progression (Prochaska \& DiClemente, 1983), however, it has become evident that movement through the stages is typically characterized by a cyclical pattern of relapses and progressions (Marshall \& Biddle, 2001; Prochaska \& DiClemente, 1992).

The key tenet of the TTM is that behavior change is a dynamic process that involves motivational readiness incorporated into a temporal dimension that is related to the three major factors (processes of change, decisional balance, self-efficacy) hypothesized to influence movement through the stages (Marshall \& Biddle, 2001; Prochaska et al., 2002).

The original hypothesis (Marcus \& Simkin, 1993; Prochaska \& DiClemente, 1983; Prochaska \& Velicer, 1997b) stated that the cognitive processes of change may be 
the most important to move through the earlier stages of change, while the behavioral processes may become most important later in the behavior change process. This relationship between stage and processes of change may vary by health outcome, however as demonstrated in a meta-analysis on the processes of change for different health behaviors (Rosen, 2000). Rosen (2000) found that in exercise adoption and diet change research, the behavioral processes of change increased together during the progression through the stages of change, as compared to smoking cessation, for which the relationship between stage and process of change adhered to the original hypothesis. To further cloud the issue, Marcus and colleagues (1992) conducted a validation study of the processes of change, and found that the correlation between the cognitive and behavioral processes of change was .91 , which actually argues against the use of a two factor solution in physical activity.

Self-efficacy and decisional balance findings in the exercise literature have been more consistent with the original TTM hypotheses. Exercise self-efficacy increases across the progression through the stages of change, while pros increase and cons decrease, with the "cross-over" point (the point at which the pros overtake the cons) coming at the transition from the preparation to the action stage (Marcus, Rakowski, \& Rossi, 1992; Marshall \& Biddle, 2001; Plotnikoff, Hotz, Birkett, \& Courneya, 2001; Prochaska et al., 2002). The original TTM hypothesis was that the increase in selfefficacy should be linear across stage progression; however, there is evidence of a nonlinear increase for exercise, indicating the potential presence of moderators (Marshall \& Biddle, 2001). Despite this, self-efficacy has a strong, consistent relationship with stage advancement. It has even been postulated that individuals may be able to be 
differentiated by stage simply on ratings of exercise or physical activity self-efficacy (Marshall \& Biddle, 2001), which calls into question the characterization of the TTM as a stage model. One criterion for a stage model is that the stages are independently characterized by the constructs contained in the full model. In other words, movement between each of the stages should require a unique set of strategies and barriers that distinguishes that stage from others (Weinstein, Rothman, \& Sutton, 1998). This is in contrast to a continuum model, where the likelihood of transition from inactive to active falls somewhere along a continuum of probability from 0 (no likelihood) to 1 (100\% likelihood).

Finally, though one of the strengths of the TTM is its inclusion of a temporal dimension for classifying the physical activity behavior change process, it is possible that the 6-month increments used in the original model are specific to smoking, and may be different based on the health behavior in question. When using the TTM in physical activity research, the transition from the active to the maintenance stage, for example, is primarily a function of time, that is, remaining active for at least six months. Though this time frame is consistent based on the literature related to drop-out (Dishman, 1988), many exercise interventions are 12 weeks in duration, which is designed to address a time period associated with physiological changes that typically occur within that period (Kearney, Stull, Ewing, \& Strein, 1976; Pollock et al., 1998). Positive physiological changes, such as increased endurance and decreased fatigue are potentially reinforcing outcomes that could factor into an individual's decision to remain active, and may occur in a shorted period than 6-months (Kearney et al., 1976; Pollock et al., 1998). Therefore, 
the 6-month time frame should be examined to determine if it is consistent with the hypothesized changes in processes of change, self-efficacy, and decisional balance.

Whether because of the complexity of the model or the difficulty in recruiting a sufficient sample size for analysis, the complete TTM has not been well tested. In the initial adaptation of the TTM to exercise, Marcus and colleagues completed a series of studies validating instruments for measuring the constructs contained in the model (Marcus, Banspach et al., 1992a; Marcus, Rakowski et al., 1992; Marcus, Rossi et al., 1992; Marcus, Selby, Niaura, \& Rossi, 1992; Marcus, Simkin, Rossi, \& Pinto, 1996). All but one of these studies were cross-sectional, and on balance did not examine the dynamic nature of exercise behavior change, only the characteristics of individuals falling into the varying stages of change.

Plotnikoff et al. (2001) examined the TTM in a population sample over a one year follow-up with no behavioral intervention, to mimic the method used in the development of the TTM in the smoking literature. Measures of all TTM constructs were taken at baseline, 6-months, and 12-months. The authors reported support for approximately 45\% of their predictions, which were based on the original TTM model. They examined respondents in terms of having either progressed, remained, or regressed stages of exercise behavior change. This allowed the authors to test whether the constructs in the model were stable in the range of naturally occurring behavioral change situations.

Despite the shortcomings of the TTM, the dynamic and temporal nature of the model makes it intuitively attractive to the health promotion practitioner. In addition, the TTM addresses a primary shortcoming of many other models, specifically, that motivational readiness is likely a moderator of exercise behavior change. It is unclear 
whether interventions that focus on behavior change as a continuum of influence from target variables will be successful, and therefore, the testing of all constructs contained within the model over time would assist in understanding optimal intervention strategies for using the model in exercise promotion. Additionally, an examination of the theoretical constructs during the 6-month time course for exercise may yield insight into the dynamics of behavior change over this critical time period.

The purpose of this study, therefore, was to test the application of the TTM (stages of change, processes of change, self-efficacy, decisional balance) to exercise behavior change by collecting data at three time points (baseline, 3-months, and 6months) in a randomly selected worksite sample of apparently healthy adults. Moderateand/or high-intensity exercise were chosen, rather than the more global level of physical activity because of evidence indicating that stage of change measures can be less reliable and valid when examining lower-intensity activities (Leslie, Johnson-Kozlow, Sallis, Owen, \& Bauman, 2003; Schumann, Estabrooks, Nigg, \& Hill, 2003). Differences in the constructs were identified by the nature of stage transition, specifically, those participants who started in an "inactive" stage of change (i.e. precontemplation, contemplation, preparation) and either progressed to an "active" (i.e. action or maintenance) stage of change or remained in an inactive stage of change by the 6-month follow-up. 
Hypotheses

All hypotheses were based on the original TTM as proposed by Prochaska and DiClemente (1983). Specifically, these hypotheses are:

o Six-month changes in cognitive processes of change will significantly predict inactive to active stage transition from baseline to six months.

o Six-month changes in behavioral processes of change will significantly predict inactive to active stage transition from baseline to six months.

o Six-month changes in decisional balance will significantly predict inactive to active stage transition from baseline to six months.

o Six-month changes in self-efficacy will significantly predict inactive to active stage transition from baseline to six months.

o Baseline to three-month and three-month to six-month changes in the above variables will not be significant predictors of inactive to active stage transition from baseline to six months. 


\section{Limitations}

The limitations for this study are listed below:

1. This study utilized a randomly selected, volunteer sample.

2. This study utilized self-report survey instruments to collect data.

3. Subjects were contacted via email and office mail for invitations to complete the online survey. Therefore, only subjects who had correct email addresses and campus mail addresses received the invitation.

4. This study was web-based. Therefore, only subjects who had access to the web at work, home or another location were able to complete the survey.

5. The environment in which the participants completed the survey was unsupervised, and therefore the participants could complete the survey where and when they were able.

6. Cut-points used in the linear regression analysis were determined using a thorough search of the TTM literature. Despite this, the criterion value that was chosen for each variable may have had an impact on the findings of the study.

7. Collapsing the stages of change into "inactive" and "active" stages may have limited the findings of this study, as the TTM was designed to be used with distinct stages.

8. The overwhelmingly white, married, educated, and active sample may have affected the findings of the study, as a sample more that was more representative of individuals from different demographic backgrounds may have resulted in different findings. 
9. The low response rate may have limited the findings by limiting the power for finding significant results.

10. The survey was completed between September and March when the weather in Ohio is relatively cold and wet. This may have limited the availability of exercise options for the participants, and therefore limited the findings of the study. 


\section{Delimitations of the Study}

The following delimitations were set by the researcher:

This investigation included only employees at the participating worksite in Columbus, Ohio, between 18 and 65 years of age, who reported a health status that was sufficient to participate in regular exercise. As such, the results may not be generalizable to other types of worksites or other regions. 


\section{Definition of Terms}

The following terms are defined to clarify their use in the study:

Decisional balance: An individual's relative weighing of the pros and cons of changing the target behavior. In this study, decisional balance was measured in two dimensions: the "Pros" and the "Cons" of exercise behavior change. A global decisional balance score was computed as the difference between these two subscales (Marcus, Rakowski et al., 1992)

Exercise Self-Efficacy: Exercise-specific confidence that one has in the ability to cope with high-risk situations without relapsing to physical inactivity. In this study, selfefficacy was computed as a mean score from a valid, reliable five-question self-efficacy scale (Marcus \& Simkin, 1993).

Regular, moderate intensity exercise: Structured, planned physical activity that is continuous, mildly elevates heart rate, mildly elevates breathing rate, but conversation is still possible while exercising, and occurs 3 or more times per week, for at least 20 minutes per session. For this study, regular, moderate intensity exercise was operationalized as weekly minutes spent participating in moderate exercise, and was measured using a valid, reliable 7-day recall instrument measuring self-reported minutes of exercise (Petosa, 1995).

Regular, vigorous exercise: Structured, planned physical activity that is continuous, causes the heart to beat rapidly, causes rapid, deep breathing, does not allow conversation while exercising, and occurs 3 or more times per week, for at least 20 minutes per session. For this study, regular, vigorous exercise was operationalized as 
weekly minutes spent participating in vigorous exercise, and was measured using a valid, reliable 7-day recall instrument measuring self-reported minutes of exercise (Petosa, 1995).

Processes of change: Strategies and techniques people use as they progress through the different stages of change over time. For this study, processes of change were operationalized as a mean score across measures of two subscales (listed below), which are comprised of all 10 processes of change. A valid, reliable processes of change instrument based on a 5-point likert scale was used (Marcus, Rossi et al., 1992; Norman, 1998).

Cognitive (experiential) processes of change: This construct is defined as relevant information about behavior change that is generated by an individual's own actions and experiences, and is comprised of the following five processes of change (Prochaska et al., 2002).

Consciousness raising: Increased awareness about the causes, consequences, and cures for a particular problem behavior (Prochaska et al., 2002).

Dramatic relief: Initially produces increased emotional experiences followed by reduced affect if appropriate action is taken (Prochaska et al., 2002).

Environmental Re-evaluation: Combines both affective and cognitive assessments of how the presence or absence of a personal habit effects one's social environment (Prochaska et al., 2002).

Self-re-evaluation: Combines both cognitive and affective assessments of one's self-image with and without a particular unhealthy habit (Prochaska et al., 2002). 
Social liberation: Requires an increase in social opportunities or alternatives (Prochaska et al., 2002).

Behavioral processes of change: This subscale is defined as relevant information about behavior change that is generated by environmental events, and is comprised of following five processes of change (Prochaska et al., 2002):

Counterconditioning: Requires the learning of healthier behaviors that can substitute for problem behaviors (Prochaska et al., 2002).

Helping relationships: Combine caring, trust, openness, and acceptance as well as support for healthy behavior change (Prochaska et al., 2002).

Contingency management: Provides consequences (punishments, rewards) for taking steps in a particular direction (Prochaska et al., 2002).

Self-liberation: Both the belief that one can change and the commitment and recommitment to act on that belief (Prochaska et al., 2002).

Stimulus control: Removes cues for unhealthy habits and adds prompts for healthier alternatives (Prochaska et al., 2002).

Stages of Change: A series of temporally-based categories that divide individuals based on readiness to change. Each stage was operationalized as follows and assessed using a five-choice algorithm (Reed et al., 1997):

Precontemplation: No regular exercise ( 3 or more times per week for at least 20 minutes each time), and no plans to start in the next six months.

Contemplation: No regular exercise ( 3 or more times per week for at least 20 minutes each time), but plans to start in the next six months. 
Preparation: Exercise below criterion level (3 or more times per week for at least 20 minutes each time), but plans to start regular exercise at criteria level within the next 30 days.

Action: Regular exercise at criteria level (3 or more times per week for at least 20 minutes each time) for less than six months.

Maintenance: Regular exercise at criteria level (3 or more times per week for at least 20 minutes each time) for six months or more. 


\section{CHAPTER 2}

\section{REVIEW OF LITERATURE}

\section{Introduction}

Much public funding and media focus have been placed on the benefits of a physically active lifestyle, however, exercise has proven to be a difficult activity to adopt and maintain (Sallis \& Owen, 1999). Many health behavior theories have been proposed and subsequently used to attempt to explain the factors that may influence the decision to become physically active and the subsequent engagement in the behavior. These have included social cognitive theory (Bandura, 1991), the theory of reasoned action (Fishbein \& Ajzen, 1975), the theory of planned behavior (Ajzen \& Madden, 1986), and the health belief model (Janz \& Becker, 1984), among others. These theories generally place people into one of two categories, either "active" or "inactive," and are conceptualized as continuum-based, or predictive of behavior by combining variables into an equation, resulting in a probability of action for the individual in question (Weinstein et al., 1998). The likelihood of action then falls somewhere along a continuum from a probability of action that equals 0 (no chance), to a probability of 1.0 (or $100 \%$ likelihood).

In contrast to these "continuum" models of behavior, the transtheoretical model (TTM) posits that health behavior does not change in such a fashion that it can be 
predicted by one equation for all individuals. Rather, health behaviors likely change through a series of stages, each stage having a combination of factors that mediate movement to subsequent stages (Prochaska \& DiClemente, 1983). Additionally, the TTM uses temporally-based staging, in which the time spent in a particular stage is defined. The TTM also introduces the concept of behavioral intention, which has been found to be another important aspect of health behavior change (Marshall \& Biddle, 2001). Behavioral intention is represented by portions of the stage definitions (see Chapter 1), which specifically cite intention (i.e., Precontemplation: No regular exercise and no plans to start in the next six months). As it is commonly accepted, the TTM consists of 5 stages of change (Precontemplation, Contemplation, Preparation, Action, and Maintenance), 10 processes of change (generally divided into 5 behavioral and 5 experiential processes), decisional balance (pros and cons of changing the behavior), and self-efficacy.

The TTM has been extensively used in the promotion of health behavior (e.g. Jordan, Nigg, Norman, Rossi, \& Benisovich, 2002; Sutton, 2001), and is an intuitively appealing behavioral model because of its "roadmap" for staging individuals and for designing an intervention to affect the corresponding mediating variables. Many interventions and cross-sectional studies have been conducted that provide some support for the efficacy of the TTM in exercise (Cardinal, 1997a; Dunn et al., 1998; Marshall \& Biddle, 2001; Prochaska et al., 1994; Rodgers, Courneya, \& Bayduza, 2001; Sarkin, Johnson, Prochaska, \& Prochaska, 2001), however, there is a relative lack of longitudinal and predictive research to support the model in this domain (Marshall \& Biddle, 2001). 
The following sections will serve to review the model's development, its use in exercise behavior research, and the criticisms leveled against it.

\section{Stage Theories}

Stage theories have emerged as a popular method for examination of health behaviors, particularly over the past 15 years (Weinstein et al., 1998). Certainly, one of the reasons for the popularity of stage theories is the variety of correlates that exist for explaining and predicting health behavior. This has led to the search among health practitioners and researchers alike for a more parsimonious list of intervention targets. If the variability among the target population can be reduced by identifying key points along the pathway to behavioral adoption, intervening to change behavior becomes a more focused and efficacious endeavor.

Some of the most commonly used health behavior theories in the health literature are social cognitive theory (Bandura, 1991), the theory of reasoned action (Fishbein \& Ajzen, 1975), the theory of planned behavior (Ajzen \& Madden, 1986), and the health belief model (Janz \& Becker, 1984). These are continuum theories, which means that they identify a host of variables that are entered into a prediction equation for the behavioral target (Oldenburg, Glanz, \& Ffrench, 1999; Weinstein et al., 1998).

In a review of stage theories applied to health behaviors, Weinstein et al. (1998) discuss four requirements of a stage model. First, there must be a classification system to define the stages. This means that each stage must have a specific set of attributes that set it apart from other stages. Second, the stages must have an order. Individuals must be able to progress from one stage to the next, working toward the behavioral goal. This is not to say that everyone will progress linearly through the stages, but that there should be 
an identifiable order of progression. Third, the barriers to individuals in a particular stage should be common to that stage. This would indicate that these individuals could be aided by an intervention targeting their particular set of barriers. Finally, people in different stages should have different barriers to change. If everyone had an equal chance to be affected by an intervention, regardless of their stage, the stage theory would be unnecessary. Several stage models have been examined in the context of exercise behavior, including the health action process approach (Schwarzer, 1992), the precaution adoption process model (Weinstein, 1988), the multi-stage model of health behavior change (Lippke \& Ziegelmann, 2006), and the transtheoretical model (TTM) (Prochaska \& DiClemente, 1983; Prochaska \& Velicer, 1997b). The TTM has been the most validated in exercise behavior. The following section describes the development of the TTM, and subsequent sections review the TTM in the context of exercise behavior.

\section{Development of the TTM in Health Behavior}

The TTM was developed using an integration of other psychological and behavioral theories (thus the "trans" portion of the moniker), from which 10 processes of change emerged (Prochaska \& DiClemente, 1983; Prochaska \& Velicer, 1997b). These processes drew from the various traditions of psychotherapy and behavior change (Prochaska \& Velicer, 1997b). For example, the consciousness raising process of change, which is defined as "finding and learning new facts, ideas, and tips that support the healthy behavior change," comes from the Freudian tradition of psychotherapy, or the extended exploration of the self (Prochaska et al., 2002; Prochaska \& Velicer, 1997b). Another example of this is the contingency management process of change, in which the individual increases rewards for positive behavior change and decreases rewards for the 
unhealthy behavior. This strategy comes from the Skinnerian tradition of behaviorism, in which antecedents and consequences are critical considerations in explaining and modifying behavior (Buckworth \& Dishman, 2002; Prochaska et al., 2002; Prochaska \& Velicer, 1997b)

The processes were subsequently examined among 872 subjects in Rhode Island and Texas who were changing their smoking habits on their own (self-changers) (Prochaska \& DiClemente, 1983). Several important findings emerged from this work. First, the stage construct surfaced as investigators examined the use of the 10 processes of change and how subjects were applying these processes to aid in changing their smoking behavior (Prochaska \& DiClemente, 1983; Prochaska \& Velicer, 1997a). This is in contrast with the understanding of practitioners and prominent researchers alike, who often view the TTM solely in light of its staging criteria (Bandura, 1997; Prochaska $\&$ Velicer, 1997a), while the stages emerged from an analysis of process use.

Second, it was found that once the stages were identified, those in the newly named precontemplation stage used 8 of the 10 processes of change less frequently than any other group. This made conceptual sense, since individuals in who are classified in the precontemplation stage of change are not motivationally ready for the target behavior, and are therefore not using any techniques to change.

Finally, consciousness raising and self-reevaluation were used more often in the contemplation stage; self-liberation, helping relationships, and reinforcement management were used more often in the action stage; and counterconditioning and stimulus control were used most often in the action and maintenance stages of behavior change. Consciousness raising and self-reevaluation are both cognitive-based processes, 
which would be expected to have a greater impact in the contemplation stage (plans to start exercising in the next 6 months), since it is an inherently cognitive stage of change and does not involve participation in the target behavior at any level. Most of the processes used in the action and maintenance stages are behavioral, and involve the individual taking some actions. This is also consistent with the change in the target behavior associated with the action and maintenance stages of change. A later study by Prochaska, Velicer, DiClemente, and Fava (1988) confirmed the two-factor model of processes of change using confirmatory factor analysis on process of change data with 970 subjects in all five stages of change. The authors called these factors 'experiential' and 'behavioral' processes of change because of the common themes among the processes.

It is generally accepted that movement through the stages of change is not a linear process, but a cyclical one, with individuals progressing and regressing on their journey to a physically active lifestyle (Marshall \& Biddle, 2001; Prochaska \& Velicer, 1997a, 1997b). This idea of nonlinear movement through the stages is important to understanding the model, as change is viewed as a dynamic process, with a different set of processes to target for individuals in each stage who are attempting to change the target behavior. If someone is not successful in applying stage-associated processes, then he or she can remain in the current stage or regress to an earlier stage.

Over time, other health behaviors, such as alcohol and substance abuse, mammography, and exercise were examined using the TTM, with results that were generally supportive of the model (Herrick, Stone, \& Mettler, 1997; Prochaska et al., 2002; Prochaska \& Velicer, 1997b). Two comparison studies illustrate initial support for 
the TTM in a range of health behaviors. A cross-sectional study of 393 employees at a municipal government worksite examined the decisional balance and self-efficacy constructs of the TTM as they related to exercise, protection from sun exposure, smoking, and dietary fat consumption (Herrick et al., 1997). Results indicated that decisional balance and self-efficacy were significantly different across the stages of change, but not across health behaviors. The crossover point for pros and cons occurred between the preparation and action stages for all four health behaviors, which supports an assumption of the TTM that there is a shift toward positive perceptions about the target behavior as the individual moves closer to adoption of that behavior. In addition, self-efficacy was higher in action and maintenance stages than in the precontemplation, preparation, and action stages, which is also a key tenet of the TTM (Prochaska et al., 2002).

In another examination of the TTM across health behaviors, 12 health behaviors were examined for generalization of the stages of change and decisional balance constructs (Prochaska et al., 1994). Twelve separate convenience samples were used, and included the following behaviors: smoking cessation, quitting cocaine, weight control, high-fat diet choices, adolescent delinquency, safer sex, condom use, sunscreen use, radon gas exposure, exercise acquisition, mammography screening, and physicians' practices. The total sample size for all 12 behaviors combined was 3,858 .

Principal components analysis on decisional balance revealed two resultant categories, which were identified as 'pros' and 'cons' in 11 of the 12 behaviors. For the high-fat diet sample, the results were somewhat ambiguous, with both a two- and fourfactor solution possible. These results provide evidence for the utility of the decisional balance measure across these 12 health behaviors. A cross-sectional analysis was also 
performed, where raw scores for pros and cons were converted to T-scores. This analysis revealed that in all 12 samples, cons were higher than pros at the precontemplation stage. In 11 of the 12 samples, pros were higher than cons in the action stage (quitting cocaine was the exception). The results for examinations of the crossover point for the pros and cons were more variable. In seven of the twelve behaviors, the crossover occurred during the contemplation stage, while for sunscreen use, high-fat diets, mammography screening, and exercise acquisition, the crossover occurred between the preparation and action stages.

Critiques of the TTM have arisen, as well. In a review of the TTM in studies of substance abuse, Sutton (2001) argues that staging algorithms are based on arbitrary time periods, and are therefore flawed. He also notes that there is little agreement between staging algorithms and questionnaires that utilize multiple dimensions to classify behavioral stage of change. Factor analyses on the multidimensional instruments revealed fewer "stages" than are postulated by the TTM. These factors were labeled "taking steps" (action and maintenance items), "recognition" (precontemplation and determination/preparation items), and "ambivalence" (contemplation items).

Furthermore, correlations between stages were often high, indicating that the instrument may not be measuring qualitatively distinct constructs.

It is important to note that each health behavior has its own set of behavioral and physiological characteristics, which creates issues regarding the assumptions about similarity among determinants. For example, it could be argued that the physiological addiction that creates difficulty in quitting smoking is quite different than the 
physiological exertion of exercise that some people find to be aversive. Similar issues will be addressed further in the TTM critique section later in this chapter. Initial Application of the TTM to Exercise Behavior

Much of the work related to applying the TTM to exercise began with a series of worksite-based studies by Marcus and colleagues in the early 1990s (Marcus, Banspach et al., 1992a; Marcus, Rakowski et al., 1992; Marcus, Rossi et al., 1992; Marcus, Selby et al., 1992; Marcus \& Simkin, 1993). In these studies, the researchers attempted to validate the aforementioned TTM constructs in the realm of exercise behavior.

In a cross-sectional examination of the processes of change, Marcus, Rossi et al. (1992) found a 10 factor model for exercise, with two hierarchical factors (behavioral and experiential processes of change) among a worksite sample of 1172 employees . At issue, however, was that the correlation between the two higher-order constructs was .908 , indicating a high degree of overlap. This calls into question the presence of two factors, since they were found to be so highly correlated. Despite this seeming contradiction to the existence of two higher-order constructs, evidence was found that indicated, like the smoking-based TTM, precontemplators used the processes the least, and that experiential processes were higher in the earlier processes of change, and peaked in the action stage, while behavioral processes of change were reported more in the later stages of change (action and maintenance). These findings provide initial evidence that it may be possible to generalize TTM constructs, as developed in the smoking cessation literature, to exercise behavior.

In another cross-sectional worksite study, Marcus, Selby et al. (1992) examined self-efficacy in relation to the exercise stages of change. Three separate studies were 
conducted to develop a self-efficacy instrument for exercise behavior change. The first study involved 1063 employees at a Rhode Island government agency, 77\% of whom were male, with an average age of 41 years. This sample was primarily ( $77 \%)$ blue collar. The second study involved 429 employees of a Rhode Island medical center, $85 \%$ of whom were female, and who had an average age of 41 . Only $38 \%$ of this sample was blue collar workers. The third study was a small sample used for instrument reliability. The following discussion will center on the first two studies.

In the first two studies, ANOVA revealed that self-efficacy was able to successfully differentiate subjects in the varying stages of change, and that self-efficacy tended to increase as stage of change improved. This is consistent with much of the research related to self-efficacy, which shows this to be one of the most consistently predictive determinants of exercise behavior (Marshall \& Biddle, 2001; Sallis \& Owen, 1999).

Another worksite sample was used to assess decision making for exercise, using a "pros vs. cons of exercise" scale (Marcus, Rakowski et al., 1992). Subjects in this study were 1,173 male and female employees at four worksites. Fifty-four percent of the sample was female, $70 \%$ was white collar workers, and the average age was 41.5 years. Data were collected by surveying at the worksites.

ANOVA results revealed that the pros of exercise behavior change increase as the stage of change moves closer to behavioral adoption, while the cons decrease. The two decisional factors "cross" each other at the preparation stage, where there is a balance between the positive aspects of the behavior change and the negative aspects. As one 
would expect, the results indicated that the pros significantly outweighed the cons for those individuals in the action and maintenance stages.

One of the studies in this series by Marcus and colleagues was longitudinal in design, and examined stage of change along with the processes of change over time (Marcus et al., 1996). Subjects were 314 employees at two worksites. The average age was 41 years, $66 \%$ were women, and $40 \%$ were blue-collar workers. Processes of change and stage of change questionnaires were administered at baseline and at a 6-month follow-up. Subjects were classified as behavioral adopters, relapsers, stable-sedentary, or stable-active. It was found that the stable groups did not exhibit any significant change in process use over the 6 month time period, while the adopters significantly increased and the relapsers significantly decreased their use of the processes of change. This was consistent with the both the TTM and the study hypotheses. Interestingly, relapsers were found to have significant decreases in all behavioral processes of change, and in only one of the cognitive processes of change. This has important implications for the importance of maintaining behavioral skills training in long-term exercise behavior change.

Overall, this series of studies provided support for using the TTM with exercise behavior; however, only one of the studies examined the activity of the TTM variables longitudinally. In a review of conceptual and methodological issues related to stage theories, Weinstein et al (1998) identified and critiqued several methodologies by which stage models could be examined. These methodologies were cross-sectional comparisons of people in different stages, longitudinal prediction of stage transitions, experimental studies of matched and mismatched interventions, and examination of stage sequences. The following sections will highlight the issues of staging within the context of exercise 
and physical activity behavior, specifically, the effects of the behavioral definition of exercise on staging. The TTM and exercise literature will then be examined in light of Weinstein's first three methodological categories, which reflect a primary aim of explaining the predictors of transition from an inactive to active stage of change. Staging in Physical Activity and Exercise - Role of Behavioral Definition

One of the primary issues with staging individuals is the behavioral definition that is used as a criterion for the behavior in question. This makes exercise unique compared to health behaviors such as smoking, where there is a clear outcome (no smoking) in terms of desired behavior. With exercise, there are minimal levels of intensity, frequency, and duration that have an impact on the desired outcome. Traditional exercise recommendations for health and fitness improvement involved a higher intensity of exercise 3-5 times per week (ACSM, 1990). More recent recommendations suggest that health benefits can result from more moderate forms of activity, performed on most days of the week (US Department of Health \& Human Services, 1996). This shift in focus resulted in a re-examination of the behavioral definition that was used in the validation of instruments for studying the TTM, and attempts to validate the measures for forms of physical activity of a more moderate intensity.

An early example of the examination of behavioral definition and method of staging is a three-part study testing the components of several staging algorithms for usefulness in exercise research (Reed et al., 1997). This study included three parts, each examining several of eight total algorithms for stage of change. In the first study, 936 working adults completed a total of four types of algorithms. The first type consists of "Ladder" algorithms, modeled after the smoking algorithm that is used in TTM research. 
The second type was an algorithm comprised of six statements in reference to stage (precontemplation to maintenance, as well as one question about relapse). Respondents rated each statement on a 5-point Likert scale. This algorithm used a short definition of exercise and a behavioral criterion of three times or more per week for 20 minutes or longer. No mention of exercise intensity was made in this definition. The third type is an algorithm that also had a short definition of exercise and used the same questions as the previous algorithm, except this version combined the action and maintenance stage questions. The fourth type of algorithm contained five true or false questions, where various stages require differing patterns of true and false responses. This algorithm used a long, detailed definition of exercise with examples of vigorous activities.

Study two consisted of 19,212 working adults who completed an algorithm that contained one short definition of exercise with no mention of intensity, and five choices to place the respondent in one of the stages of change. Study three participants $(N=327)$ completed three algorithms as well as measures of pros and cons, confidence, and hours of exercise. The first algorithm was nearly identical to the previous long definition-true false version, with one minor change in the first question. The second algorithm used the long, vigorous definition of exercise and the five choice staging method, and the third algorithm contained a definition of exercise that was more consistent with lifestyle, moderate activity recommendations, and used the five choice staging method.

The authors found that the ladder staging method tended to stage more participants in contemplation and the Likert-scale method tended to stage more toward preparation. When comparing the Likert scale to the five-choice method, it was found that the five-choice method shifted participants into the extreme stages of 
precontemplation and maintenance. The short definition of exercise tended to produce higher numbers of individuals in the lower stages of change, where the longer definition of exercise tended to produce higher numbers in the preparation and action stages. The authors concluded that the ambiguity of the short definition caused participants to set a lower standard, while the long definition allowed for more accurate comparison of one's behavior to the criteria. For the lifestyle versus vigorous definition comparison, it was found that a greater percentage of participants classified themselves in the maintenance stage using the lifestyle definition as compared to the vigorous definition. The authors concluded that this validated the common assumption that lifestyle exercise criteria are easier to meet than vigorous exercise criteria. It was also determined that the classification into the preparation stage was the least reliable among the measures, likely because of the ambiguous definition of this stage. In fact, some definitions for preparation have included a time frame for intention to meet the behavioral criterion (e.g. "within the next month"), and other definitions have not.

In the end, it was concluded that the algorithm with the long, vigorous exercise definition and five choices for staging produced the closest to the "classic" pattern of staging based on decisional balance, self-efficacy, hours of exercise, and thus is recommended for use in examinations of TTM constructs. The primary issue with using this definition, however, is the inability to examine stages of change as they apply to more moderate activities as recommended for health benefits from exercise (Pate et al., 1995).

Two different studies have specifically examined the validity of stages of change based solely on exercise intensity. The first of these studies used four different data sets 
consisting of participants of varying age groups (Schumann et al., 2002). The first was an adolescent sample of 400 respondents with an average age of 17.6 years $(S D=1.18)$, the second was a college student sample of 240 respondents with an average age of 20.3 years $(S D=6.20)$, the third was an adult sample of 346 respondents with an average age of 42.8 years $(S D=14.9)$, and the final group were 504 seniors with an average age of $74.5(S D=6.8)$. Participants completed a measure of stage of change in which exercise was "defined as any planned physical activity performed three to five times per week for 20 minutes or more at an intensity that increases a person's breathing rate and causes him or her to break a sweat" (Schumann et al., 2002 p. 282). Participants in the first three groups also completed the Godin Leisure Time Exercise Questionnaire (Godin \& Shepard, 1985). The senior participants received different measures of exercise behavior which assessed the frequency of aerobic exercise and similar activities as well as the frequency of walking. MANOVA was used to test for stage differences on strenuous, moderate, and mild exercise (as measured by the Godin instrument), bivariate correlations were used between stage and exercise intensity to assess the magnitude of the relationship, dependent t-tests were used to examine any increase in correlations from mild to moderate to strenuous exercise, and lastly the authors examined whether the stage instrument differentiated people in the "inactive" stages of change (precontemplation, contemplation, and preparation) from those in the active stages (action and maintenance) for each level of exercise intensity as measured with the Godin instrument.

MANOVA analyses revealed stage differences for strenuous and moderate exercise, specifically, those in the "active" stages reported significantly more exercise per week than those in the "inactive" stages for strenuous and moderate definitions, but not 
for mild. Analyses also revealed stepwise increases in strenuous and moderate exercise across the stages, but not for mild. Correlations between mild exercise and stage of change were also found to be significantly smaller than correlations between moderate and strenuous exercise and stage of change. These findings suggest this stage algorithm is valid for use with strenuous and moderate exercise, but not for mild. One of the primary issues with generalizing these findings to all algorithms was the definition of exercise used in this study. Because mild exercise was defined as "minimal effort, no sweating..." (Schumann et al., 2002 p. 282), one would not expect that it would be highly correlated with a stage measure that defined exercise as of an intensity that increased breathing and caused sweating. Despite this limitation, the study provides some interesting insight into the behavioral criteria used in a staging algorithm.

Another study out of the same lab attempted to validate stages of change in varying intensities of exercise using 467 adult respondents (Schumann et al., 2003). In this study, regular physical activity was defined as "any planned physical exertion aimed at improving or maintaining physical fitness and health, performed at least 3 times per week for at least 20 to 30 minutes at least moderate intensity" (Schumann et al., 2003 p. 364). The Godin Leisure Time Exercise Questionnaire (Godin \& Shepard, 1985) was again used in this study, as were measures of intention and self-efficacy.

Analyses revealed that moderate exercise, as measured by the Godin instrument, successfully distinguished between the stages of change, while strenuous exercise along with intention to participate in strenuous exercise and self-efficacy for strenuous exercise distinguished between stages. For mild exercise, only intention to perform mild exercise and self-efficacy to perform mild exercise distinguished between stages. These findings 
serve to further highlight the problems with using stages of change to examine the TTM in mild intensity exercise. Some activities that are recommended in the lifestyle-oriented activity guidelines (Pate et al., 1995) would likely be classified as mild in the Godin instrument, and therefore may not perform as theorized when using one of the accepted and validated staging algorithms.

A study by Sarkin, Johnson, Prochaska, and Prochaska (2001) also attempted to validate a stage of change measure with an overweight sample of 670 otherwise healthy adults. Participants completed measures of stage of change using a behavioral definition of regular moderate exercise that was planned, performed five to seven days per week for 20 to 40 minutes per day, and intended to increase fitness. Participants were reminded that as defined, exercise does not have to be painful or exhausting, but that it would be sufficient to increase breathing rate and cause a sweat. Participants also completed the Godin Leisure Time Exercise Questionnaire (Godin \& Shepard, 1985), as well as measures of decisional balance and self-efficacy.

Tests of concurrent validity revealed significant differences in strenuous exercise between stages of change, with participants in the action and maintenance stages having more sessions of strenuous exercise than those in precontemplation, contemplation, and preparation. For moderate exercise, the mean amount of exercise was found to be below the criterion level, but the authors concluded that the staging algorithm may capture all activity moderate and above, so a moderate and strenuous summary score was computed, which resulted in those participants in the action and maintenance stages having activity levels above the criteria, as well as significantly higher activity levels than those in the other stages. The decisional balance scores and self-efficacy scores also were 
significantly different across stages, providing construct validity with the theorized relationships. Though this study did not report all results for mild exercise, analyses also revealed that those in the maintenance stage performed significantly more mild exercise than those in precontemplation stage. This study provides more evidence that traditional definitions of exercise that are used in staging algorithms may be useful for moderate and strenuous activity, but there is a gap in the literature relative to mild exercise.

In another study of behavioral definition and staging, young adults in the United States $(N=105)$ and Australia $(N=123)$, completed a questionnaire measuring stage of change for moderate and vigorous activity at two time points, separated by one and two weeks, respectively (Leslie et al., 2003). For the participants in the United States, moderate activity was defined as physical activity performed for 30 or more minutes on five or more days of the week, with examples given such as walking briskly, digging in the garden, and vacuuming. Vigorous activity was defined for these participants as activities such as power walking, jogging, swimming, etc. done for at least 20 minutes, three times per week. Australian participants were given a definition of "recreational or industrial activity such as walking and cycling five times a week for at least 30 minutes each time," (Leslie et al., 2003, p. 179) and a definition of vigorous activity as “...vigorous physical activity such as sport or fitness activities (e.g. aerobics and running) three times a week for at least 20 minutes each time" (Leslie et al., 2003, p. 179). No clear reason was given for the differing behavioral definitions used in the American and Australian samples. Test-retest repeatability was examined between the first and second administrations of the instruments. 
Analyses revealed that the vigorous-intensity activity definitions produced significantly better test-retest reliability scores than the moderate intensity definitions in both the American and Australian samples. Leslie et al. (2003) concluded that this may have been a function of the definition, and that these moderate activities may not be as salient in the thinking of young adults as vigorous intensity activities, and therefore information about these activities may not be as reliable from one time point to the next. Also, the vigorous definition on the American staging measure used the term "exercise," while all of the other measures used the term physical activity. The exercise definition produced the highest reliabilities, and the authors hypothesized that perhaps the term "exercise" was better understood by the participants than "physical activity," and therefore resulted in higher reliability scores.

Another study has taken this line of inquiry and applied it to other cultures and associated modes of physical activity (Miilunpalo, Nupponen, Laitakari, Marttila, \& Paronen, 2000). Specifically, these authors were interested in validating a stage of change measure in outdoor aerobic exercise and everyday commuting activity in a Finnish population. Interestingly, the authors of this study found that their subjects could be in different stages of change for each of the types of activity, which they concluded emphasizes the importance of a specific, well-defined behavioral criterion for examining changes in stage relative to the TTM.

In earlier research on the TTM in exercise, many studies examined the model in the context of a rather ambiguous behavioral definition for exercise. As a result of the findings reviewed here, increasing numbers of studies that have tested the TTM in exercise use a more specific behavioral definition for exercise. It is important to note, 
however, that many of the activities in lifestyle physical activity recommendations fall into the "mild" category, for which there has been limited support relative to staging individuals. A review by Nigg (2005) highlighted the inherent difficulty in operationally defining milder forms of exercise as a possible cause of this limited support. Nigg (2005) also identified empirical evidence that specificity and sensitivity for classifying individuals into stages using mild exercise is lacking. Future studies must be conducted to generate a valid algorithm for staging mild exercise in order to aid in identifying intervention targets for advancing stage of change.

\section{Cross-Sectional Examinations}

By far the largest body of research in model testing of the TTM has been crosssectional in nature. Cross-sectional examinations of theory have been cited as a weak test of the constructs and processes involved with the TTM (Weinstein et al., 1998), yet these designs are among the most prevalent in claims of the usefulness of the TTM in the literature. The examination of cross-sectional studies is a logical place to begin looking at the validation of the model, but these studies provide only preliminary information about its utility because of the limitations of the design.

Many of the original cross-sectional studies applying the TTM to exercise were conducted by Marcus and associates, and were reviewed in the previous section (Marcus, Banspach et al., 1992b; Marcus, Rakowski et al., 1992; Marcus, Rossi et al., 1992; Marcus, Selby et al., 1992; Marcus \& Simkin, 1993). The primary purpose of these studies was instrument development, though they were useful in describing the characteristics and tendencies of the TTM constructs as applied to exercise behavior. There have been many other cross-sectional studies used to establish the validity of the 
TTM in exercise behavior, and most have focused on validating a measure of TTM constructs or establishing the validity of the TTM in exercise with various populations, including youth, young adults, working adults, older adults, adults with disabilities, lowincome groups, and international populations.

Some of the first cross-sectional studies to examine the TTM in the context of exercise focused on young adults. In a study of 244 young adults in Britain, participants completed a questionnaire containing items concerning exercise stage of change, exercise self-efficacy, self-esteem, and exercise behavior (Wyse, Mercer, Ashford, Buxton, \& Gleeson, 1995). The primary purpose of the study was to establish concurrent validity of the exercise stages of change using the Godin Leisure Time Exercise Questionnaire (Godin \& Shepard, 1985). A secondary purpose was to examine the ability of selfreported exercise behavior, BMI, self-efficacy, and self-esteem to discriminate between the exercise stages of change.

It was found that males tended to be in a more advanced stage of change than females, as $56.4 \%$ of male respondents were classified in the action/maintenance stages as compared to $36.3 \%$ of female respondents. Respondents in the action/maintenance stage reported significantly higher levels of exercise than those in the preparation stage, and those in the preparation stage reported significantly higher levels of exercise than those in the precontemplation/contemplation stage. Additionally, self-efficacy was found to be significantly higher for those in the action/maintenance stage as compared to those in the precontemplation/contemplation stage. Self-esteem scores were not found to be significantly different between stages. These results provided partial preliminary support for the stages of change, as the behavioral measure was able to distinguish between those 
in the various stages combinations, and the self-efficacy construct of the TTM was also significantly different between stage combinations. This support was tempered by the cross-sectional nature of the design and analysis, as well as the lack of inclusion of all TTM constructs. The combination of stages is an important limitation, as it does not allow the results to be applied fully to intentional differences, but only the behavioral differences between stage combinations.

In another study of young adults, Pinto and Marcus (1995) examined the stages of change construct as applied to college students. Two hundred seventeen respondents completed a questionnaire containing measures of exercise stage of change, interest in health topics, physical activity, dietary intake, medical services use, and demographic information. It was found that individuals in the precontemplation and contemplation stages had no difference in activity levels (both were inactive) so those stages were combined. Combining individuals with different intentions based on behavior alone was a limitation noted in the previous study, as well. No data were available on the length of time during which participants had been active, so no one was classified in the maintenance stage of change. It was found that $28 \%$ of individuals were in the precontemplation/contemplation stage, $28 \%$ were in the preparation stage, and $54 \%$ were in action. There were no significant differences found between men and women, and year in school was not associated with exercise stage of change. Results from this study were primarily descriptive, but at minimum provided concurrent validity between exercise stages of change and a measure of exercise behavior, as well as indicating that there was no interaction between exercise stage of change and age (year in school) or gender. 
In a study of the TTM in adolescent exercise behavior by Nigg and Courneya (1998), 819 high school students were given a questionnaire to assess demographic data, exercise stage of change, processes of change, self-efficacy, and decisional balance. This was one of the first studies to examine the TTM in its entirety. The authors hypothesized that experiential processes of change would be higher in the contemplation stage compared to the precontemplation stage, and higher in the action stage compared to the preparation stage. The use of environmental (behavioral) processes of change was hypothesized to be greater at each stage from precontemplation to action, and then level off at the maintenance stage. Self-efficacy and pros were hypothesized to increase across all stages of change, and cons to decrease. Finally, the pros and cons were hypothesized to cross-over at the preparation stage. These hypotheses are in line with theorized relationships between the TTM constructs and stage.

Corrected univariate ANOVA revealed that each construct differentiated at least one stage of change from another, and the largest effect sizes $\left(\omega^{2}\right)$ were seen for the counterconditioning and self-liberation processes of change, and for self-efficacy (.257, .141 , and .237 respectively). The authors found through MANOVA that the pros and cons crossed over in the action stage, and hypotheses related to the experiential and behavioral processes of change as well as self-efficacy were also generally supported. Overall, support for the hypothesized relationships between TTM constructs was found, and the primary strength of this study was the use of the entire TTM model.

In a study validating a stage of change measure in an overweight population, 654 adults with a BMI $\geq 25$ completed measures of stage of change, situational confidence (self-efficacy), processes of change, and decisional balance for exercise and for three 
other health behaviors (Sarkin et al., 2001). Data regarding time spent exercising were also collected using the Godin Leisure Time Exercise Questionnaire (Godin \& Shepard, 1985). The authors did not hypothesize any relationships between TTM constructs and the stages of change, as this was simply an assessment of concurrent and construct validity of a stage of change measure assessing moderate exercise behavior.

The authors found good content validity for their measure as determined through significant ANOVA results on differentiation of stage by time spent exercising using the Godin Leisure Time Exercise Questionnaire, and good construct validity by observing a significant linear increase in confidence by stage of change as well as a significant standard deviation (SD) increase in pros from the precontemplation to the action stage, and a half-SD decrease in cons from precontemplation to action. This indicates support for the cross-over point of the decisional balance constructs, which is the point at which the perceived benefits of the health behavior outweigh the perceived barriers. No results were reported for processes of change. Certainly, the results of this study provide some evidence of consistency with the proposed relationships between several TTM constructs and the stages of change, though the lack of any longitudinal data or data relative to the processes of change limits the authors' ability to draw any conclusions about dynamic movement between stages.

A study examining gender differences in TTM constructs across three health behaviors also provided some insight into the gender differences and the TTM $\left(\mathrm{O}^{\prime} \mathrm{Hea}\right.$, Wood, \& Brantley, 2003). Five-hundred thirty seven of a total sample of 554 medical patients were healthy enough to participate in exercise and completed questionnaires 
relating to exercise stage of change, exercise self-efficacy, and exercise decisional balance while attending a medical appointment at one of the testing sites.

Chi-square analyses revealed that there were gender differences in stage of change membership, most notably there were significantly more females than males in the contemplation stage, and significantly more males than females in the maintenance stage. T-tests also revealed that males had significantly lower overall scores in both pros and cons when compared to females. Self-efficacy differences between the genders were not found to be statistically significant, though there was a trend of males having higher self-efficacy scores than females. Though this study provided some insight into where gender differences might lie in a few TTM constructs, the overall contribution to understanding stage of change progression was limited, as a consequence of the types of analyses that were conducted, and the cross-sectional nature of the study.

Few studies have examined all of the constructs contained within the TTM, even using cross-sectional design. The following studies report data on the complete model, in addition to data on the combination of physiological variables and self-reported measures of physical activity in order to provide further validation for the stages of change with respect to exercise.

In a validation study of a stages of change questionnaire (Dannecker, Hausenblas, Connaughton, \& Lovins, 2003), 152 university students completed measures of the stages of change, processes of change, decisional balance, and self-efficacy. Participants also completed a maximal treadmill test to determine aerobic capacity. The maximal treadmill test and self-reported measures of exercise behavior were included as part of the validation of a stages of change instrument. The authors hypotheses were in line with the 
proposed TTM, that is, that cognitive processes of change would be greater at each progressive stage, behavioral processes of change would be greater at each progressive stage but equal between contemplation and preparation, self-efficacy would be greater at each progressive stage but equal between preparation and action, pros would be low at precontemplation, higher at contemplation, and higher at each progressive stage, with no difference between action and maintenance, and cons would be high at precontemplation, lower at contemplation, and each progressive stage, with no difference between action and maintenance.

MANOVA and subsequent discriminant analysis revealed that both sets of processes of change, self-efficacy, and pros were the best predictors for distinguishing early (Precontemplation, Contemplation, Preparation) versus late (Action, Maintenance) stages of change, with the behavioral processes of change seeming to be the strongest predictor. Additionally, ANOVA revealed significant between group differences by stage in all TTM constructs, with post-hoc testing finding only partial support of the hypothesized differences. Dannecker et al (2003) concluded that these differences could have been the consequence of using discriminant analysis as a post-hoc test for the MANOVA results as opposed to separate ANOVAs. They also reported that those in the later stages had higher levels of self-reported strenuous exercise than those in the early stages, and that those in the later stages also had higher levels of self-reported moderate exercise than those in either precontemplation or contemplation. Participants in the maintenance stage had higher levels of fitness as measured by the maximal treadmill test. Findings of this study also indicate partial support for the hypothesized constructs within the TTM. 
In a study of physically active adults, Buckworth and Wallace (2002) examined differences in the psychosocial variables contained within the TTM and physiological variables among young adults classified in either the action or maintenance stage of change. Participants were 57 young adult men and women who were enrolled in a collegiate conditioning class. At one meeting, participants were given measures of stages of change, exercise self-efficacy, decisional balance, processes of change, and exercise behavior. Participants were also assessed at the same meeting for maximal aerobic capacity and heart rate/blood pressure. The authors' hypotheses were based on the TTM, that is, that there would be no difference in exercise self-efficacy or pros, that cons would be lower among those participants in the maintenance stage, and that the cognitive processes of change would be higher in action than in maintenance, and that behavioral processes of change would not be different between the groups. Also, it was hypothesized that self-report of exercise and physical activity would not be different between stages, and the physiological variables associated with regular participation in exercise (heart rate, blood pressure, maximal aerobic capacity) would differ as a function of the length of time that the participants had been active.

ANOVA revealed that compared to participants in the action stage, individuals in the maintenance stage had significantly greater differences in the pros and cons of exercise, significantly greater use of the consciousness raising process of change, and significantly greater use of the counterconditioning, self-liberation, and stimulus control processes of change. No significant differences were found in any other constructs between the two stages, though resting systolic blood pressure and $\mathrm{VO}_{2 \text { peak }}$ were both higher in those participants classified in the maintenance stage of change. Again, these 
findings provide only partial support for the TTM in exercise, and corroboration of the stage construct with the observed differences in aerobic capacity. In contrast to modelsupporting findings, the finding that participants in the maintenance stage endorsed more cognitive processes of change than those in action runs counter to the theorized relationship between these variables.

Several more recent studies have used the cross-sectional design in an attempt to provide initial validation of the TTM in other populations. A study by Rodgers, Courneya, and Bayduza (2001) examined TTM constructs in 168 high school students, 215 university undergraduate students, and 63 working adults. The authors were interested in studying the TTM as it applies to differing populations within one study, as opposed to the conclusions that had previously been drawn from differing populations in different studies. Participants completed a questionnaire measuring exercise stage of change, processes of change, and self-efficacy. The authors hypothesized that there would be a main effect for stage, the possibility of a main effect for population (indicating consistent differences by population across all of the measured constructs of the TTM), and no interaction effects. The authors pointed out the importance of this last hypothesis, as an interaction effect would indicate that TTM principles would not be generalizable across populations.

ANOVA revealed significant main effects for stage for all processes of change, self-efficacy, and population main effects for eight of the ten processes of change and for self-efficacy. No significant stage by population interactions existed for any of the variables. Post-hoc testing revealed that those participants in the precontemplation stage reported using the processes of change less than those in other stages, and the use of the 
behavioral processes of change increased as the stages of change moved closer to maintenance. The working adults reported the lowest use of the processes of change, while university students were reported the highest. These findings provide initial support that the hypothesized patterns of change in the TTM apply across age-based populations, as there was no significant interaction and any differences among stages in this study were consistent.

In another attempt at validating a portion of the TTM, Blue, Black, Conrad, and Gretebeck (2003) examined the TTM in a population of blue-collar workers. The authors combined the stage and self-efficacy aspects of the TTM with the Theory of Planned Behavior (Ajzen, 1991) to examine the variability in exercise stage of change in this population. Four hundred sixty-eight maintenance, building, and grounds workers in a university setting completed a questionnaire containing Theory of Planned Behavior (TPB) constructs as well as items relating to demographics, self-efficacy for exercise, exercise stage of change, intention, and the Godin Leisure Time Exercise Questionnaire. Blue et al. (2003) described the primary purpose of the study as providing evidence for the combined contribution of constructs from various behavioral theories for explaining exercise behavior. A secondary purpose was to describe differences in the measured variables by stage of change.

LISREL modeling revealed that self-efficacy had a direct influence on stage of change, and was also indirectly linked through TPB constructs. For example, in the authors' final model, the variance in attitude and perceived behavioral control (TPB constructs) that was explained by self-efficacy was $67 \%$ and $41 \%$ respectively. Univariate ANOVAs with associated post-hoc testing revealed significant differences in 
self-efficacy by stage in every instance except between action and maintenance. Though it was not the primary purpose of this study to fully test the TTM, the findings relative to self-efficacy seem to provide initial support for the theorized relationship that selfefficacy increases linearly with advancing stage.

Also in the context of working adults, Herrick, Stone, and Mettler (1997) examined stages of change, decisional balance, and self-efficacy in exercise, sun protection, smoking, and fat consumption among 393 government workers. Participants completed a questionnaire measuring demographic variables as well as the previously mentioned TTM constructs for all four health behaviors. The authors cited as their purpose to compare the number of participants in each stage between health behaviors, to compare those participating in ongoing worksite health promotion programs with those not participating, and to examine reported levels of decisional balance and self-efficacy across the health behaviors and stages of change.

Data were analyzed using MANOVA and follow-up ANOVA methodologies, which revealed significant differences in numbers of participants in each stage of change across the different health behaviors, and only $18.6 \%$ were in the same stage of change for all four health behaviors. Interestingly, all of those $18.6 \%$ were in the maintenance stage. There were also significant differences between wellness program participants and non-participants on stage of change for exercise and dietary behaviors, but not for smoking or sunscreen use. The authors also found that self-efficacy and decisional balance-pros were significantly higher, and decisional balance-cons were significantly lower in the action and maintenance for all health behaviors as compared to the precontemplation and contemplation stages. The cross-over point for decisional balance- 
pros and decisional balance-cons was between the preparation and action stages. Though there were no longitudinal data to support the importance of the measured TTM constructs in movement between stages, again some evidence was found that is consistent with the theorized relationships between the stages and the remaining TTM constructs.

One series of cross-sectional studies out of Cardinal's lab examined the full TTM model in active, inactive, and a mix of active and inactive adults with physical disabilities (Cardinal, Kosma, \& McCubbin, 2004; Kosma, Cardinal, \& McCubbin, 2004; Kosma, Gardner, Cardinal, Bauer, \& McCubbin, 2006). Study 1 in the series was an examination of 322 primarily active participants with a variety of physical disabilities (Cardinal et al., 2004). Participants completed a survey containing measures of stage of change for exercise (modified for a disabled population), processes of change for exercise, selfefficacy for exercise, and decisional balance for exercise, along with exercise barriers. The authors hypothesized that self-efficacy and behavioral processes of change would be the primary differentiating variables for stage of change for exercise, as had been previously reported in health adult populations. The authors were also interested in examining the overall relationship between the stages of change and all TTM variables, as no previous study had been conducted that included all constructs with disabled adults.

Univariate analyses revealed that the majority (53.7\%) of participants was in the maintenance stage of change, and all constructs (decisional balance, all processes of change, and self-efficacy) were significantly associated with exercise stage of change. The largest effect sizes were seen for the behavioral processes of change and selfefficacy. Use by stage generally followed the expected theoretical patterns, with selfefficacy increasing with advancing stage, pros and cons crossing over between the 
preparation and action stages, behavioral processes of change used more in later stages, and cognitive processes of change used more in the contemplation and preparation stages. Discriminant function analyses including all TTM constructs revealed an overall stage classification probability of $70.8 \%$. These results indicate good agreement with the TTM, and cross-sectional evidence that the TTM may be useful in a disabled population.

Study 2 in the series again targeted adults with disabilities, but this time participants were screened to be in one of the early stages of change (precontemplation, contemplation, or preparation), and able to exercise despite their disability (Kosma et al., 2004). Participants completed an internet-based survey of all TTM constructs. The authors again hypothesized that the relationship between exercise stages of change and the remaining TTM constructs would follow theorized patterns.

A few participants were classified in the action and maintenance stages, but most reported being in the early stages of change. MANOVA revealed that there were significant associations between stage of change and all other TTM variables, with general trends of increasing use of processes of change, and increasing self-efficacy with advancing stage. The few participants who classified themselves in the maintenance stage of change reported significantly higher pros and lower cons than those in other stages of change. Discriminant analyses revealed that the primary predictors for stage of change were the behavioral and cognitive processes of change $\left(\mathrm{r}^{2}=.88\right.$ and .50 respectively), followed by self-efficacy $\left(\mathrm{r}^{2}=.33\right)$ and decisional balance $\left(\mathrm{r}^{2}=.13\right)$. The precontemplation stage was the most accurately predicted stage at $76.3 \%$, followed by preparation (58.3\%) and precontemplation (40\%). Action and maintenance were not accurately predicted in the analysis (.0\% and $8.3 \%$, respectively). Although the measured 
TTM variables were unable to predict the active stages in this population, there is some cross-sectional support for theorized relationships between constructs.

The third study in the series examined both active and inactive adults with disabilities (Kosma et al., 2006). The previous two studies had samples that were skewed toward either active or inactive participants, thus the authors were hoping to replicate the previous findings in a more representative sample. Two hundred twenty four participants completed an online survey measuring stage of change, processes of change, selfefficacy, decisional balance, and physical activity recall.

Participants were distributed throughout the stages of change, though only $3.6 \%$ classified themselves in the action stage, while $35.7 \%$ classified themselves in the maintenance stage. Because of this, the authors combined the action and maintenance stages of change for the analysis. MANOVA revealed a linear association between the behavioral processes of change and the stages of change, and a nearly linear association between the cognitive processes of change and stages of change. Like in the previous two studies, participants in the action and maintenance stages of change perceived significantly more pros and fewer cons than those in other stages of change. Self-efficacy was found to increase across the stages, but the change in magnitude did not indicate a linear increase. Discriminant analysis revealed that only the behavioral and cognitive processes of change predicted membership in the inactive stages versus membership in action/maintenance, as well as differentiating between those in the precontemplation stage and those in the preparation stage. Overall stage classification accuracy was 52.7\%, with the action/maintenance stage being the most accurately predicted. It was also found that the behavioral and cognitive processes of change were highly correlated, providing 
support for examining the processes of change as a single factor. The findings of this study were similar to the previous two, though the role of self-efficacy in predicting stages was found to be not as strong, while the processes of change were found to be stronger predictors. As a whole, these three studies provide some support for the application of the TTM to individuals with disabilities, and for the theorized relationships between TTM constructs.

Some studies have also used the cross-sectional design to examine the TTM as it applies to older adults. One such study by Gorely and Gordon (1995) examined the TTM in exercise with 50-65 year olds in Australia. The entire model was included in this study. Five-hundred eighty-three participants completed a questionnaire measuring stages of change, self-efficacy, processes of change, and decisional balance. Analyses revealed two cognitive processes of change and three behavioral processes of change that discriminated between stages. These processes increased from precontemplation to contemplation, and from preparation to action. Self-efficacy was found to significantly increase across stages from precontemplation to maintenance, and pros significantly increased across stage while cons significantly decreased. Overall, the findings are consistent with previous research in the TTM in exercise, and serve to provide initial support for the use of the model with older adults.

Another study with older adults combined TTM and Social Cognitive Theory constructs in a cross-sectional examination of 179 adults aged 65 or older (Resnick \& Nigg, 2003). Participants completed a questionnaire containing measures of self-efficacy, outcome expectations, exercise behavior, health status, fear of falling, and stage of change. Structural equation modeling revealed that the factors that directly influenced 
physical activity behavior were self-efficacy, outcome expectations, and stage of change. Any effects shown through other variables were exerted through self-efficacy, outcome expectations, and stage of change. The final model explained $70 \%$ of the variability in exercise behavior. These results provide more evidence for the importance of the stages of change and self-efficacy in changes in exercise behavior. The analyses were more advanced in this study than in many of the previously reviewed studies, which allowed the researchers to estimate a model that explained the maximum amount of variance.

A few other cross-sectional studies have examined the TTM in relation to other populations, and found varying results. A study by Fahrenwald and Walker (2003) examined the full TTM in the context of 30 WIC mothers, and found significant relationships between most TTM variables and stage of change along the hypothesized lines. Participants in the later stages of change used the behavioral processes significantly more than the other processes of change, and self-efficacy increased across all stages of change. Unique pros (sense of accomplishment, stress relief) and cons (childcare) were found that influenced the behavior among the WIC mothers in this study.

In another study of a low-income population, 554 low-income adults attending public primary care clinics were given measures of exercise stage of change, selfefficacy, and decisional balance (Carmack-Taylor, Boudreaux, Jeffries, Scarinci, \& Brantley, 2003). Self-efficacy was significantly different across all stages of change, with those in the later stages of change having higher levels of self-efficacy. The pros were significantly less important for those in precontemplation than all other stages, while the cons were more important for those in contemplation than all other stages. One of the issues that was reported in both this population and the WIC mothers were the 
examples used in pros/cons and self-efficacy. Unique socioeconomic issues with this population could have a stronger bearing on perceived pros and cons of exercise, which could have affected the results in both of the studies. The stress of struggling for family survival create a unique set of barriers for these populations, which further reinforces the need to consider moderating variables (such as income and family dynamics) in exercise behavior change initiatives. Further examination of these factors was recommended by Fahrenwald et al. (2003) and Carmack-Taylor et al. (2003).

Two separate studies were identified that attempted to apply the TTM in nonEnglish-speaking populations (Cardinal, Tuominen, \& Rintala, 2003; Tung, Gillett, \& Pattillo, 2005). Both found at least partial support for the TTM in their respective populations. In a study of family caregivers in Taiwan, support was found for the selfefficacy construct in TTM (Tung et al., 2005). Specifically, self-efficacy was significantly higher in the later stages of change than in the earlier stages, though no differences were found for pros or cons. In a psychometric assessment of TTM constructs translated into Finnish, self-efficacy and the behavioral processes of change were the only significant predictors of stage of change as analyzed using regression analysis (Cardinal et al., 2003). Percentages of variance explained were by these constructs were $34 \%, 18 \%$, and $26 \%$ respectively in the Finnish, American, and combined samples.

Many of the issues with cross-sectional study design were summed up nicely in a meta-analysis conducted by Rosen (2000). In her meta-analysis, she focused on the processes of change across the stages in various health behaviors including smoking, exercise, substance abuse, diet, and psychological problems. Rosen (2000) found that 
processes of change vary by health behavior, and recommended that the relationship between processes and stage that exist in one health behavior should not be assumed to hold for other health behaviors. Specific to exercise, it was pointed out that both categories of processes of change have been shown to be used sequentially as stage advances. Rosen even concluded that a stage model may not be appropriate for exercise given these findings, and that a continuum model may be better suited for a behavior that requires active participants to use both cognitive and behavioral strategies to maintain it. Rosen also pointed out that the only true method to answer the questions that arise from cross-sectional studies such as those previously reviewed in this section is to examine those questions prospectively.

Despite some findings that support the application of the TTM to exercise, the cross-sectional nature of these studies calls into question the validation of the model in exercise. The model should be tested in individuals who are naturally changing their exercise behavior, much like the initial TTM research in smoking self-changers. Unfortunately, fewer such studies have been conducted with somewhat mixed results.

\section{Longitudinal Designs and Prediction}

Longitudinal studies allow a more dynamic view of the changes in TTM constructs that correspond with shifts in exercise stage, as they provide more than a 'snapshot' comparison of the static differences among stage members. Another benefit of the longitudinal study is that it could allow for an examination of the temporal aspect of the TTM. No non-intervention studies were identified that examined the importance of the 6-month period in stage of change and TTM construct change, however. All but one of the following studies examined the TTM over a period of 6 months or longer, and 
the study that utilized a shorter time frame looked at the predictive power of baseline stage for stage at 1 and 3 months, and drew no conclusions about the time course of change (Cardinal, 1997b).

One of the first studies that used a longitudinal design to test the TTM in exercise was conducted by Armstrong, Sallis, Hovell, and Hofstetter (1993). Participants completed a survey at baseline and at a two-year post-baseline follow up that contained measures of interest in exercise, self-efficacy, and participation in vigorous exercise. The authors staged participants based on their self-reported interest in exercise. For example, participants whose lack of interest "often" or "very often" prevented them from exercising were placed in the precontemplation stage, and those whose lack of interest "rarely" or "never" prevented them from exercising were placed in the contemplation stage (Armstrong et al., 1993 p. 394). Participants also marked during which months they participated in regular exercise, so that the authors could identify the levels of participation six months after the baseline measurement. It was hypothesized that those in the contemplation stage would report more participation in vigorous exercise by sixmonths post-baseline than those in the precontemplation stage, that those in the contemplation stage would have higher levels of baseline self-efficacy than those in precontemplation at baseline and that those in contemplation at baseline would be more likely to advance to the action or maintenance stage by six-months post-baseline than those in precontemplation at baseline, and that stage of change at baseline would be a significant predictor of participation in exercise at the six-month post-baseline follow-up after controlling for baseline self-efficacy. The authors hypothesized that baseline self- 
efficacy would differ by baseline stage, so controlling for self-efficacy would help to sort out the predictive ability of baseline stage of change.

Analyses revealed that baseline contemplators had significantly higher levels of baseline self-efficacy than precontemplators, and contemplators reported a significantly higher participation in exercise by 6-months post-baseline than those in precontemplation. In addition, multivariate analysis revealed that participants' baseline stage was a significant predictor and accounted for $13 \%$ of the variance in exercise participation at 6-months post-baseline, even after controlling for baseline self-efficacy, age, and gender. Finally, $46 \%$ of those in contemplation at baseline progressed to action or maintenance at 6 months post-baseline as compared $24 \%$ of precontemplators. These findings provided some intriguing preliminary support for the TTM at the time, partly because of the use of a prospective research design. The method that was used to stage individuals (not self-reported criteria but assumptions based on interest in exercise) creates problems in drawing solid conclusions related to the findings because validated measures of stage were not used. This study served as a springboard, however, to more controlled longitudinal research on the TTM.

Cardinal (1997b) conducted another longitudinal examination of the role baseline stage of change in plays in predicting the transition to adoption and maintenance of regular exercise. Sixty six females at a worksite who were classified in the contemplation or preparation stages of change at baseline completed surveys containing demographic information, the Godin Leisure Time Exercise Questionnaire (Godin \& Shepard, 1985), as well as a measure of exercise stage of change at baseline, 1-month post-baseline, and 7-months post-baseline. Cardinal (1997b) hypothesized that, after 
controlling for baseline levels of exercise behavior, those participants in the preparation stage at baseline would have significantly higher levels of exercise behavior than those in contemplation by 7 -months post-baseline, and that those in preparation at baseline would improve in exercise stage to a greater degree than those in contemplation at baseline.

ANCOVA revealed that the first hypothesis was not supported. Specifically, there was no significant difference in exercise participation (measured in METs) at 7-months post-baseline between those classified in contemplation and those in preparation at baseline. Though exercise behavior at follow-up was not found to be significantly different by baseline stage, linear regression revealed that demographic data and baseline stage of change were significant predictors of stage improvement at 1-month and 7months post baseline. Interestingly, those in contemplation at baseline were found to be more likely to improve their stage of change by 7 -months than those in preparation at baseline, which contradicted the second hypothesis. Cardinal (1997b) used a validated measure of stage of change in this study, which allowed for a clearer picture of stage movement between time-points than reported by Armstrong et al (1993), who decided upon stage assignment based on self-reported interest in exercise. Though Cardinal (1997b) did not test all of the TTM components, the longitudinal design allowed more insight into the process of stage transition and demonstrated the inadequacy of baseline stage alone as a predictor of transitions in stage and exercise behavior.

Wallace and Buckworth (2003) examined the relationship between naturally occurring exercise stage of change transition and psychosocial variables over a 6-month follow-up in 173 college-age students. Participants completed a survey containing measures of demographics, stage of change, self-efficacy, family and friend social 
support, and sedentary behavior at both time points. Participants who advanced from an inactive to an active stage did not show significant changes in any of the psychosocial variables, however, those participants who regressed from an active to a non-active stage showed decreases in variables such as peer social support and exercise self-efficacy. Despite finding no relationship between advancing stage and psychosocial variables, these findings further highlight the importance of longitudinal research in understanding the more intricate details of exercise behavior change and stage transition.

A more recent longitudinal study examined the entire TTM model and the change in exercise stage as it naturally occurred over both a 6- and 12-month follow-up (Plotnikoff et al., 2001). Plotnikoff et al. (2001) conducted 638 telephone interviews of adults in the Ottawa-Carleton region of Ontario, Canada, at baseline, 6-months, and 12months. Participants responded to questions about stage of change, self-efficacy, pros, cons, processes of change, and demographic information. The behavioral definition for exercise in this study was vigorous physical activity, or "strenuous activities which usually make you sweat, breathe harder, and feel your heart beat at least three times per week for at least 20 minutes per time" (Plotnikoff et al., 2001 p. 443). Participants in the action and maintenance stages were combined for the data analysis because of the small sample size $(N=3)$ in the action stage. Participants were classified into the following categories in reference to their baseline stage of change: Regressed, remained, or progressed. Participants were categorized as regressed if they moved backward at least one stage, as remained if they did not change stage, and progressed if they advanced at least one stage. The two consecutive 6-month time periods were analyzed separately to save statistical power. Partial support for the TTM was found. Of the 40 possible 
predictions (based on the TTM), only 45\% were supported, with self-efficacy being the strongest and most consistent predictor of stage transition. Predictions based on the processes of change constructs were inconsistent with the TTM model. Importantly, the behavioral processes of change were found to be instrumental in stage transition in the pre-action stages, in contrast with the hypothesized importance of the cognitive processes of change. The authors recommended further examination of the interrelationships between the independent TTM constructs (processes of change, decisional balance, selfefficacy), as well as the integration of further concepts from other social-cognitive models.

In a study of predictors of stage progression and regression conducted by Higgins \& Oldenburg (2003), 238 female university students completed measures of stage of change, self-efficacy, decisional balance, perceived behavioral control, affect, and desire to exercise at baseline and 6-months post-baseline (Higgins \& Oldenburg, 2003). The authors were interested in examining the strongest predictors for change in stage between baseline and 6-months post-baseline.

At follow-up, $37.7 \%$ of participants had progressed from an inactive stage (precontemplation, contemplation, or preparation) to an active stage (action or maintenance). Logistic regression revealed that baseline levels of self-efficacy and decisional balance were not significant predictors of stage progression at follow up, while change scores in those two variables were significant predictors of progression. Once both self-efficacy and decisional balance were entered into the overall model, however, decisional balance failed to remain a significant predictor of progression. Additionally, changes in perceived behavioral control and baseline desire to exercise were significant 
predictors of stage progression. Though some support was found for TTM constructs predicting change in stage, it is difficult to draw complete support because stages were collapsed in defining stage progression (i.e. inactive to active stage) and not all of the TTM variables were measured.

In another longitudinal study, the role of self-efficacy in stage movement was examined in the context of exercise, smoking cessation, and fat reduction (O'Hea et al., 2004). Participants were 554 adult patients attending an appointment at primary medical care clinic. Measures included stage of change for each health behavior, self-efficacy for each health behavior, and demographic information. Participants were contacted one month later for collection of data on the same variables.

Change in stage from baseline to 1-month post-baseline according to baseline stages of change and baseline self-efficacy scores for each health behavior were examined. Participants in the lowest baseline tertile for self-efficacy were predicted to regress. Those in the middle tertile for self-efficacy were predicted to be stable, and those in the upper tertile were predicted to progress. Chi squared analyses compared predicted to observed (actual) regressors, stable participants, and progressors. Observed progressors and regressors were required to have changed by at least one stage between baseline and follow up. For exercise, $50 \%$ of predicted progressors actually progressed a stage, and $7 \%$ regressed a stage. Of those predicted to progress, $29 \%$ actually regressed, but $29 \%$ progressed a stage. There were several methodological problems with this study. First, prediction was measured with an arbitrary assignment of tertiles of selfefficacy. Also, Chi-squared analyses only allow elementary conclusions to be drawn regarding the driving factors in stage movement and differences between progressors, 
regressors, and those maintaining their stage. Another limitation was the one-month follow-up period, as the TTM and other validation research specifies 6-months as being critical to the stage transition process. Despite the flaws, this study provides some support that self-efficacy may be among the important predictors of stage transition for exercise.

Two different studies were found that conducted longitudinal tests of TTM constructs in adolescents. Nigg (2001) examined the full TTM model in Canadian high school students. Four hundred students completed baseline and 3-year follow up questionnaires measuring exercise stage of change, self-efficacy, processes of change, decisional balance, and the Godin Leisure Time Exercise Questionnaire (Godin \& Shepard, 1985). Panel analysis was used to examine which TTM variables were the driving forces behind behavior change, and which were a result of the behavior change.

In general, strenuous, moderate, and total exercise declined, as did the number of individuals classified in the later stages of change between baseline and follow-up. None of the theorized relationships between stage movement and processes of change were found to be significant. However, data did tend to show a trend toward supporting the assumption that experiential (cognitive) processes are more important in the early stage transitions, and that behavioral processes are more important in later stages. Positive changes in exercise behavior did lead to higher levels of self-efficacy, and changes in the pros and cons were also found to be significant and a consequence of changes in baseline to follow-up exercise behavior. Though this study utilized the entire TTM, its primary limitation was found in the lag time between baseline and follow-up. Most longitudinal studies that have been reviewed in this section utilized a 6-month follow up, because 6- 
months is the theorized time frame contained within the TTM. Interestingly, this study showed that changes in self-efficacy can result from changes in exercise behavior, which is different from other studies that have used the standard method of examining selfefficacy as a mechanism or predictor for behavior change.

The second longitudinal study of adolescents in this review was conducted by Maddison and Prapavessis (2006), and involved 1434 high school students in Auckland, New Zealand. The authors of this study had a similar purpose as the Nigg (2001) study, which was to examine the full TTM model longitudinally and to determine whether the TTM constructs act more as predictors or consequences of stage of change transition. The design of this study specifically addressed two methodological issues from the Nigg (2001) study. First, Maddison and Prapavessis (2006) examined the data in a 6-month time frame, compared to the 3-year follow up employed by Nigg (2001). Second, the authors of this study did not group the behavioral and experiential processes of change together, because they felt that this might mask the effects of one process of change driving the movement between stages.

Participants completed measures of stage of change, self-efficacy, processes of change, and decisional balance at baseline, and 6-months later. Cross lagged panel correlations and structural equation modeling were used to analyze the data. Results indicated that self-efficacy had a reciprocal relationship with exercise stage. That is, selfefficacy acted as both an important predictor and consequence of exercise in this sample. Additionally, reciprocal relationships were identified between decisional balance and exercise stage, and two behavioral processes of change and exercise stage. These results shed more light on the results of the Nigg (2001) study, and create speculation about the 
causal relationship between exercise stage and the hypothesized predictors of stage transition. Though the authors did not provide suggestions for future follow-up of these results, it seems likely that a stage-matched vs. stage-mismatched intervention design could answer some of the questions raised by the results of these two studies. The stagematched portion of this design involves identifying the key variables for moving individuals from one stage to the next, and basing the appropriate intervention on these variables. Individuals in other stages of change would need a different intervention based on the unique set of variables hypothesized to move to the next stage. The stagemismatched portion of this design would use an intervention that has been designed for a different stage than the one in question. Weinstein (1998) has promoted the stagematched and mismatched design as a rigorous test of the efficacy of the stage model.

The final longitudinal study to be discussed in this review was conducted by Rhodes and Plotnikoff (2006), and involved a sample of 1192 adults in the OttawaCarleton area of Ontario, Canada. This study did not use the traditional definitions of stage of change, but instead examined intention profiles. Participants completed a telephone survey and answered questions about self-efficacy, pros and cons, processes of change, past physical activity, intention, and future physical activity. The final three variables were used to form an intention profile, and to group participants into one of five categories (nonintenders, unsuccessful adopters, successful adopters, unsuccessful maintainers, and successful maintainers). The other TTM variables were used as predictors to examine the factors involved in classification in the intention profiles. All measures were taken at baseline and at a 6-month follow-up, and all questions asked were related to vigorous physical activity. 
Discriminant analysis and subsequent post-hoc testing revealed that self-efficacy and behavioral processes of change were the best predictors of intention profile. This model identified $42 \%$ of cases correctly. Successful discriminating variables were as follows: Behavioral processes significantly discriminated all profiles, and nonintenders had the lowest score while successful maintainers had the highest; self-efficacy significantly discriminated all profiles except successful adopters and unsuccessful maintainers; pros and cognitive processes significantly discriminated nonintenders from the other profiles; and cons significantly discriminated unsuccessful adopters from successful ones. When the high-order TTM constructs were disaggregated to examine potential intervention points, it was found that these disaggregated variables did not follow the same patterns as the higher-order variables. The disaggregated variables will not be identified here because of the number of these variables, and limited applicability to our study. Despite not using traditional definitions of stage of change, one of the strengths of this study was the disaggregation of the higher-order constructs of the TTM. This allows future research into the most effective targets for intervention and research into specific predictors of change in stage. Like previous studies, self-efficacy and behavioral processes of change were found to be integral in behavioral transition. This is inconsistent with the theorized relationship among the behavioral processes of change and stage membership, as behavioral processes of change have been proposed to be most important in the later stages of change (Prochaska \& Velicer, 1997b).

In recent years, the call for more longitudinal examination of the TTM in exercise has been answered. Despite the deeper data about stage transition that results from this design, there have been issues related to the validity of the findings, the definitions used 
to describe the criterion behavior, and sample sizes that limit power to test some of the effects. Additionally, these longitudinal studies examined the transition between stages in 6-month or greater intervals (up to 3 years), with no examination of the importance of intermediary changes. Only a few trends emerged regarding which of the TTM constructs are most critical in predicting the transition from inactive to active status, though self-efficacy and behavioral processes of change were identified in more than one instance as being critical variables (Higgins \& Oldenburg, 2003; Maddison \& Prapavessis, 2006; Nigg, 2001; O'Hea et al., 2004; Plotnikoff et al., 2001; Rhodes \& Plotnikoff, 2006; Wallace \& Buckworth, 2003).

There are still questions about the direction of influence, as well. It is unclear whether self-efficacy and other TTM constructs are primarily predictors or consequences of stage transition. Replication with various populations and settings has also "muddied the water" of validation, because of the introduction of moderating factors that effect results. The call remains for further longitudinal inquiry into the TTM to address these and other issues.

\section{Experimental Stage Matched and Mismatched Designs}

Another recommended method for examining the dynamics of the TTM is the stage matched and mismatched experimental design (Weinstein et al., 1998). In this design, one group of participants receives a behavioral intervention that is matched to their stage of change, while another group receives an intervention tailored to a different stage of change. Only one study was identified that used this design in exercise and physical activity behavior. Blissmer and McAuley (2002) used random assignment of 196 college employees to one of four 16-week intervention conditions. These conditions 
were comprised of a stage-matched intervention, where participants received materials via campus mail once per month that were matched to their baseline exercise stage of change; a stage-mismatched condition, where participants received the same materials that were developed for the stage-matched condition, but only received materials that differed from their baseline stage of change; a standard care condition, where every month participants were sent a randomly selected manual developed by the American Heart Association concerning physical activity; and a control condition, where participants received a new general health pamphlet every month throughout the study. Participants first received an initial questionnaire packet that contained measures of stage of change and level of physical activity, as well as other measures not included in this manuscript. Participants also received a new questionnaire at the end of each four-week period throughout the study to assess their stage of change. After participants returned this stage of change instrument, the intervention materials were sent according to their current stage of change.

Blissmer and McAuley (2002) found that baseline levels of physical activity for their participants was quite high. At baseline, $76.7 \%$ of respondents were in the action or maintenance stage at baseline, which had serious implications for the expected efficacy of the behavior change intervention. In the end, there were no significant changes in exercise behavior throughout the intervention, though decreases in total physical activity approached significance, as did increases in lifestyle activity. MANOVA revealed that there was no significant difference in stage movement by study condition, and effect sizes revealed that for total activity there were little to no changes in the stage-matched and standard care conditions, while there were small decreases in total activity in the stage- 
mismatched and control conditions. Examining lifestyle activities, there were small to moderate effect size increases in the stage-matched and standard care conditions, while the stage-mismatched and control conditions produced no change.

Blissmer and McAuley (2002) concluded that season may have had something to do with lack of significant results, as weather was reportedly cold and wet toward the end of the study as compared to the early part of the study. It would also seem that the high levels of baseline physical activity may have created a ceiling effect, which limited the ability of the intervention to show any tangible effects. The trends in the data suggested that the stage-matched intervention was better than the control and stage-mismatched, but it is difficult to draw any real conclusions from these results. Future studies may need to use purposeful sampling to ensure that there are representative numbers of participants in each stage of change at baseline, before the start of the intervention. As we learn more about the individual factors that predict stage transition, follow-up studies of this design could shed even more light on what factors are most efficacious in optimizing physical activity outcomes.

\section{Stage Matched Interventions}

A more typical application of the TTM in longitudinal research is found in stagematched intervention studies. Only a few intervention studies apply the full TTM model through measuring and targeting TTM variables beyond stage, which makes it difficult to assess the usefulness of a TTM-based intervention in changing exercise behavior. Even in intervention studies that use the processes as postulated by the TTM, it is difficult to draw any conclusions about the effectiveness of TTM based interventions on exercise behavior change unless the effects of the intervention on these mediating variables are 
measured (Baranowski et al., 1998). Exercise intervention studies based on the TTM are summarized in Table 2.1. Details about the content validity of the treatment (CVT) are included to highlight those studies that report attempts to measure the effects of TTMbased mediating variables on the outcome of interest.

Several studies have used the full TTM in an exercise intervention with moderate success (Bock, Marcus, Pinto, \& Forsyth, 2001; Dallow \& Anderson, 2003; Nichols et al., 2000; Pinto, Lynn, Marcus, DePue, \& Goldstein, 2001; Woods, Mutrie, \& Scott, 2002). In each case, the intervention had a significantly greater impact on the mediating variables (self-efficacy, processes of change) than a non-intervention control. In a few cases (Kosma, Cardinal, \& McCubbin, 2005; Loughlan \& Mutrie, 1997; Naylor, Simmonds, Riddoch, Velleman, \& Turton, 1999; Nichols et al., 2000), post-intervention measures of activity or stage of change were not different between the control and intervention groups. Interventions introduce quality of implementation and bias issues that could have an impact on the relationship between observed changes in TTM constructs and stage movement. This disparity in results from intervention studies, whether a function of methodological issues or other confounders, further emphasizes the importance of studying the model in a naturalistic sense in order to identify the key points for examination at the intervention level.

Questions also remain regarding the importance of 6-month time point as a marker of motivational readiness for behavior change in the precontemplation, contemplation, action, and maintenance stages (Weinstein et al., 1998). To date, this temporal issue has not been explicitly examined, though one intervention study did examine the prediction of six-month changes in stage based on change data from baseline 
to three months (Lewis et al., 2006). The purpose of this study was to examine the impact of an intervention based on the TTM and social cognitive theory on behavioral processes, cognitive processes, self-efficacy, and decisional balance. Participants $(N=150)$ were sedentary adults who were randomly assigned to either a tailored intervention group or a standard-care group. The intervention group received stage-matched, print-based selfhelp materials, and the standard care group received activity promotion booklets designed by the American Heart Association. Questionnaires measuring the aforementioned constructs were distributed at baseline, 1-, 3-, and 6-months post-baseline.

Lewis et al. (2006) found that few differences between the intervention group and the standard-care group, though overall positive changes in behavioral process of change use and self-efficacy from baseline to 3 months were significantly related to positive changes in minutes of physical activity at a 6-month follow-up when controlling for baseline levels of physical activity. These results also highlight the need for further inquiry into the time course of change that is required for TTM constructs in order to produce a shift in exercise stage of change. 


\begin{tabular}{|c|c|c|c|c|c|c|c|}
\hline Study Authors & Year & Subjects & $\begin{array}{l}\text { Duration of } \\
\text { Data } \\
\text { Collection }\end{array}$ & Design & $\begin{array}{l}\text { TTM } \\
\text { Outcomes } \\
\text { Measured }\end{array}$ & $\begin{array}{c}\text { CVT } \\
\text { Evaluation }\end{array}$ & Main Findings \\
\hline $\begin{array}{l}\text { Blalock, DeVellis, } \\
\text { Patterson, Campbell, } \\
\text { Orenstein, \& Dooley }\end{array}$ & 2002 & $\begin{array}{l}\text { Adult females, } \\
\qquad N=547\end{array}$ & 12 months & $\begin{array}{l}\text { Two groups: stage-tailored } \\
\text { educational materials, non- } \\
\text { tailored materials }\end{array}$ & $\begin{array}{l}\text { SOC (as } \\
\text { mediator) }\end{array}$ & No & $\begin{array}{c}\text { Inactive stage } \\
\text { members sig } \\
\text { increased PA by 3-, 6- } \\
\text {, and } 12-\text {-month follow- } \\
\text { ups }\end{array}$ \\
\hline $\begin{array}{c}\text { Bock, Marcus, Pinto, \& } \\
\text { Forsyth }\end{array}$ & 2001 & $\begin{array}{c}\text { Sedentary } \\
\text { adults, average } \\
\text { age } 39, N=255\end{array}$ & $\begin{array}{l}6 \text { months, } 12 \\
\text { month follow } \\
\text { up included } \\
\text { also }\end{array}$ & $\begin{array}{l}\text { Two groups: Stage-matched } \\
\text { materials and feedback, standard } \\
\text { treatment control (AHA booklets) }\end{array}$ & $\begin{array}{l}\text { SOC, DB, } \\
\text { POC, SE }\end{array}$ & $\begin{array}{l}\text { SOC, DB, } \\
\text { POC, SE }\end{array}$ & $\begin{array}{l}\text { Stage-matched group } \\
\text { sig higher in PA and } \\
\text { behavioral POC than } \\
\text { standard treatment, } \\
\text { both groups sig } \\
\text { increased SE, Pros., } \\
\text { behavior and cog } \\
\text { POC }\end{array}$ \\
\hline $\begin{array}{l}\text { Calfas, Long, Sallis, } \\
\text { Wooten, Pratt, \& Patrick }\end{array}$ & 1996 & $\begin{array}{c}\text { Sedentary } \\
\text { adults, average } \\
\text { age } 39, N=255\end{array}$ & $\begin{array}{l}1 \text { meeting with } \\
\text { physician; } 6 \\
\text { week follow } \\
\text { up }\end{array}$ & $\begin{array}{l}\text { Physician-based, two groups: } \\
\text { Physician trained in TTM-based } \\
\text { physician counseling, or trained in } \\
\text { HepB diagnosis and treatment }\end{array}$ & $\begin{array}{l}\mathrm{SOC} \text { at } \\
\text { baseline }\end{array}$ & No & $\begin{array}{l}\text { Increase in self-report } \\
\text { and activity monitor } \\
\text { measured PA higher } \\
\text { in patients seeing } \\
\text { TTM-trained } \\
\text { physicians }\end{array}$ \\
\hline Cardinal \& Sachs & 1996 & $\begin{array}{l}\text { Females, aged } \\
22-50, N=113\end{array}$ & 31 days & $\begin{array}{l}\text { Mailed one of three Packets: } \\
\text { Stage-matched lifestyle exercise, } \\
\text { structured exercise, control }\end{array}$ & SOC & No & $\begin{array}{l}\text { Stage-matched packet } \\
\text { most effective }\end{array}$ \\
\hline
\end{tabular}

Table 2.1 TTM-Based Exercise Intervention Studies 
Table 2.1 Continued

Dallow \& Anderson

Dunn, Marcus, Kampert,

Garcia, Kohl, \& Blair

$\checkmark$

Hager, Hardy, Aldana, \& George

Working adults average age 42 ( \pm 6.39$), N=525$

Harland, White, Drinkwater, Chinn, Farr, Howell
Sedentary, females, $N=44$

Sedentary adults aged 35-60,

$N=235$

\section{6 months}

Web-based, three groups: stage-

based materials, action stage materials, or health information control

Five groups, TTM-Brief interview only, TTM-brief interview and activity voucher, TTM-multiple interviews only, TTM-multiple interviews and voucher, no treatment control

SOC, POC, SE

SOC, POC,

SE

TTM-based group sig increased $\mathrm{POC}, \mathrm{SE}$,

PA; trad group sig increased only 2 POC

Increase in METs

greater for gym-based

program, both groups sig reduced physiological measures, increased SE, POC and DB

Action stage group sig increased leisure time PA, occupational PA, and energy expend, stage-based group only leisure time PA sig increase, no change in SE

\section{Activity scores} increased in intervention vs control but no diff btw intervention groups. 
Table 2.1 Continued

Heesch, Masse, Dunn, \&
Frankowski
$\begin{gathered}\text { Jarvis, Friedman, Heeren, } \\ \text { \& Cullinane }\end{gathered}$
Jimmy \& Martin

2005

Adults, $N=132$

Kao, Lu, \& Huang

Working adults

in Taiwan,
Sedentary adults aged 25-75, $N=244$

Sedentary,

1997 primarily wome

65 and old
$N=68$

3 months

$N=193$

1 counseling session, follow

$$
\begin{gathered}
\text { up } 7 \text { weeks } \\
\text { and } 14
\end{gathered}
$$

months later

stage-specific feedback, feedback plus advice

Two groups: TTM-based

Three groups: Prime G - stage

matched and met in groups,

Prime C - stage matched and

received info through mail and

elephone, and standard care

Two groups: Telephone TTM-
based counseling, standard medical care control

SOC
SOC, POC
DB, SE
Physician-based, two groups: plus advice

5 months no treatment control. Tested

psychosocial outcomes
Those completing twothirds of HW had

greater changes in

POC and SE than those completing less.

Counseling group who were in Contemp at

baseline had sig

higher stage than those in control

At 7 weeks, $35 \%$ of feedback and $38 \%$ of feedback + advice were active; at 14 mos $47 \%$ of Ss in both groups were active. No diff btw groups

Self-

Pros, self-efficacy significantly higher in experimental vs. control and cons significantly lower 
Table 2.1 Continued

\author{
Kirk, Higgins, Hughes, \\ Fishert, Mutrie, Hillis, \& \\ Maclntyre \\ Kosma, Cardinal \& \\ McCubbin \\ Lewis, Forsyth, Pinto, Bock, \\ Roberts, \& Marcus
}

2001

Adults with type-

$$
\begin{gathered}
2 \text { diabetes } \\
N=26
\end{gathered}
$$

Adults with

physical

2005

disabilities, av

age $38.73( \pm$

8.86), $N=75$

4 weeks

Healthy,
sedentary
adults, $N=150$

6 months, 3

months for

construct

Mediator analysis with two

groups: stage matched TTM /

SOC materials, standard care

group

$$
\begin{aligned}
& \text { Three groups: Fitness testing } \\
& \text { group, TTM-based exercise } \\
& \text { consultation group, and }
\end{aligned}
$$

adults, $N=179$
6 months
Consultation group

increased behavio

and stage of change significantly compared to info only

No significant differences in lifetime

physical activity between treatment and control

Stage matched group had sig greater

increases in behav

POC and SE from BL

$\mathrm{POC}$ and SE from BL
to 3 months compared

to standard care

No differences

between groups in PA

participation post-

intervention at 3

months, or at 6

months 
Table 2.1 Continued

Marcus, Emmons, SimkinSilverman, Linnan, Taylor Bock, Roberts, Rossi, \& Abrams

Two groups: stage-matched selfhelp intervention and standard materials (AHA materials) follow-up, stagematched more likely to show increases than those with standard materials

At 2 mos, full intervention group more likely than

1 mailing, 2

Marshall, Bauman, Owen, 2003 Adults, aged 40$60, N=462$

follow up measures
Mutrie, Carney, Blamey, Crawford, Aitchison, \& Whitelaw

Naylor, Simmonds, Riddoch, Velleman \& Turton
Working adults $64 \%$ female, aged 19-69, $N=295$

12 months

Healthy adults $77 \%$ female, average age $42.4( \pm 15.1)$, $N=294$

2 months
Two groups: intervention (mailed intervention (general TTM) packet at start) or control, outcome measures taken at BL, 6 months, 12 months

Stage-matched materials with counseling, stage-matched s without counseling, nontaged materials with counseling ontrol to meet ACSM

PA guidelines, at 6

mos intervention who

read materials more

likely than control to meet guidelines

Intervention group $2 \mathrm{x}$ more likely to increase walking, no change for cycling, SOC progression significantly higher in intervention group

Stage-matched no better than othe conditions, all in stage progression 
Table 2.1 Continued

Nichols, Wellman,

Caparosa, Sallis, Calfas, \& Rowe

Norris, Grothaus, Buchner, Pratt

2000
SOC

$$
\text { counseling }
$$

No
Adults, aged 24-

$61, N=64$

9 months

Two groups: In-person TTM-

based behavioral and activity skills group, control group (free membership to gym)

Physician counseling - two

$30+, N=812$

6 months

or normal care control

SOC, SE, DB

SOC, SE,

DB

Mod and Vig activity increase in both

groups, POC

increased more in

TTM-based group

BL Contemplators increased SOC in tage-based group stage-based group

compared with

controls, no other sig differences between groups

PA counseling group more likely to meet PA recommendatio had higher ens and had higher energy expenditure than eating group at $3 \mathrm{mos}$. Changes not adults, average $\quad 6$ months $\quad$ counseling, eating behavior age $45.9, N=298$

maintained at 6 mos 
Table 2.1 Continued

Pinto, Lynn, Marcus,
DePue, \& Goldstein

Purath, Miller, McCabe, $\&$ Wilbur

ư Riebe, Blissmer, Greene,
Caldwell, Ruggiero,

Stillwell, Nigg

Steptoe, Kerry, Rink, \& Hilton
Older adults, 1 meeting with average age 6 week, and 8 $65.6( \pm 9.1)$ $N=355$

Sedentary working adult females, $N=287$

1 brief intervention, follow up at 6 weeks

Two groups: stage based counseling brief intervention, control group who received general health counseling

All subjects received TTM-based 6-month clinical program, two groups then formed: extended care treatment (stage-matched

verweight and

obese adults,

average age

$0.2( \pm 9.2)$

2005

$N=144$

24 months

2001

Adults, $N=883$

Physician-based, two groups: Physical trained in TTM and SCT. ased physician counseling, or left to provide usual care materials), extended care control (generic materials) hysician counseling - two groups: stage-based lifestyle counseling or standard health
SOC, POC
SE, DB

SOC, POC, $\mathrm{SE}, \mathrm{DB}$

$\mathrm{DB}, \mathrm{SE}$, and beh POC sig higher in TTM group vs usual care at 6 weeks, no difference at 8 months; DB and beh POC were only mediators of motivational readiness at 6 weeks

Intervention group significantly increased PA compared to control

Changes were maintained for 24 months, those who maintained ex had sig

SOC, DB

$$
\text { promotion }
$$

Odds ratio for increasing to active 
Table 2.1 Continued

Steptoe, Rink, \& Kerry

van Sluijs, van Poppel,

Twisk, van Meechelen

๙

Woods, Mutrie, \& Scott
Sedentary

2000 adults, average

$( \pm 11.2), N=418$

2006

Adults, aged 18-

$70, N=635$

2002
Young adults,

$N=203$
12 months

Provider counseling - two groups:
stage-matched or standard health

$$
\text { promotion }
$$

Testing effect of measurement on

PA - two groups: measured at BL,

2-, and 6-months; measured only at 6 months

$\mathrm{SOC}, \mathrm{DB}$,

POC, SE

Two groups: stage-matched selfinstruction intervention, no

$$
\text { intervention }
$$

SOC increased more with stage-based counseling than

standard control at 4 months in those with

motivation to change at 12 months predicted by $\mathrm{DB}$

\section{Only TTM variable} showing sig highe levels in multimeasure group was

$$
\text { SE }
$$

Experimental group increased SOC sig more than controls, stage improvers scored sig higher on 9 of 10 POC scales 


\section{Criticisms of the TTM}

Despite the appeal of the TTM in health behavior research, there have been a number of criticisms leveled against it. First, it has been postulated that the time points associated with the stages of change set forth by the TTM are somewhat arbitrary (Weinstein et al., 1998). Weinstein and colleagues (1998) argued that if the time points were moved, the stage distribution would be altered. A call is also made for more research into the factors that differentiate changes in stage. A stage model must have different processes for movement from different stages, and similar processes for movement from the same stage.

Albert Bandura (1997) argued that a true stage model does not involve the "skipping" of stages, rather, it is comprised of an ordered set of stages through which one must pass to reach the behavioral destination. One of the assumptions of the TTM is that change can be cyclical, though whether individuals skip stages is a matter of contention among TTM researchers. Bandura (1997) also disputed the descriptive nature of intention that is included in the TTM, and that simply stating such an intention may not make the TTM a dynamic model, as it is proposed.

As mentioned previously, the cross-sectional design provides only limited information regarding the validity of theory because dynamic changes in behavior cannot be examined. Despite the use of statistical methods to examine the relationships between constructs contained within the behavioral model, it is impossible to infer causality from cross-sectional data because we are not aware of the direction in which the relationship exists (Weinstein, 2007). In other words, it is unclear from the 'snapshot' that crosssectional data provide whether the level of behavior is a result of the level of construct 
use, or the level of construct use is a result of the level of behavior. Much of the criticism of the the TTM comes from its reliance on cross-sectional validation, though this is hardly unique among behavior change theories (Weinstein, 2007).

Also in reference to cross-sectional examination, a review by Sutton (2000) discusses the usefulness of finding discontinuity patterns in cross-sectional research. Specifically, to infer that a stage model may exist, levels of constructs should not change linearly across stages but rather change in patterns that suggests that each stage is distinct, and that movement between stages does not simply require a larger does of the construct in question. Sutton (2000) references Weinstein and colleagues' (1998) discussion of pseudo-stages, where arbitrary points along a continuum of construct use and change in behavior are selected as cut-off points for each stage. This procedure is a stage-model in name only, as the stages implied by such an approach are not distinct in their predictor variables for movement to the subsequent stage.

In a critical review of the TTM in exercise, Adams and White (2003) analyzed TTM intervention studies to examine trends in the results and to determine if there were methodological issues that could be improved. The primary finding was that interventions were successful in promoting exercise behavior change in the short-term (6 months or less), but had mixed success in promoting activity long-term (more than 6 months). The authors concluded that various methodological and implementation issues could be at fault for these inconsistent and disappointing results, including lack of control over implementation, varying methods of assessing stage of change and exercise behavior, and a lack of attempt to retain individuals from underserved groups. 
Jordan, Nigg, Norman, Rossi, and Benisovich (2002) attempted to address the intention construct by incorporating a measure of attitude, which is a direct influence on intention according to the Theory of Planned Behavior (Ajzen, 1991) in their study of exercise in college students. They assessed exercise behavior and factors hypothesized to influence this behavior, including stage of change and other TTM constructs in 223 college undergraduates. It was found through structural equation modeling and discriminant function analysis that the model with the best fit to the data included two attitudinal subscales, and that the addition of these subscales increased the explained variance among the stages of change to over $50 \%$, and the predictive ability of the decisional balance scale to about $48 \%$. This indicates that it could be useful to add an intention or attitudinal construct to the TTM to increase its predictive ability.

The addition of an intention measure could also address those individuals who fall between stages in this model. It is sometimes unclear where an individual who is active, but below the criteria level should be staged. Some of these individuals fit nicely into the preparation stage because they are working toward increasing their activity to the criterion level. Others may also be exercising below the criterion level, but have no plan to increase their activity level. This is especially the case for people who engage in physical activity solely for health reasons, and are not interested in increasing their amount of vigorous physical activity to the level indicated in the stage definition.

Finally, a commentary paper by West (2005) has even called for a complete discard of the TTM in health behavior promotion. He cites the model's flawed and arbitrary approach to staging, the instability of stages (as opposed to the inferred 6-month duration of the contemplation stage), lack of a real measure of readiness to change, and 
its lack of consideration for addiction in behaviors such as smoking and substance abuse. West (2005) even contends that the popularity of the TTM in health behavior promotion has held the field back, and health practitioners and researchers alike would be better served to discard the model completely even without a suitable replacement. Though West (2005) cites the addiction literature in his reasoning for abandonment of the TTM, the criticisms leveled mirror those of the scholars of exercise behavior whose work has been reviewed here and who have been skeptical of the application of the model to exercise. Despite the time and work that researchers have put into the study of the TTM in exercise, there are still many unanswered questions about the utility of the model as a tool for exercise behavior change.

\section{Summary}

The TTM is an appealing model for predicting exercise behavior change and for designing effective interventions. Much support has been garnered for the model and its usefulness in explaining and predicting exercise behavior (Marcus, Banspach et al., 1992a; Marcus, Rakowski et al., 1992; Marcus, Rossi et al., 1992; Marcus, Selby et al., 1992; Marcus \& Simkin, 1993; Marcus et al., 1996; Plotnikoff et al., 2001; Prochaska \& Velicer, 1997b). Despite this, there are inconsistencies in the literature and a lack of convincing empirical evidence for the validity of the model applied to exercise.

Certainly, we have much to learn about the effectiveness of the constructs contained within the TTM in predicting exercise behavior, and about the moderators and mediators that most strongly influence individuals to move from inactive to active stages of change (Marshall \& Biddle, 2001). Increasing numbers of longitudinal studies are being conducted with the TTM in exercise, but the bulk of supporting data for the model's use 
in this context is derived from cross-sectional data. Furthermore, only one study was identified in exercise that uses the stage-matched versus stage-mismatched intervention design proposed to provide further insight into the effectiveness of the model (Blissmer \& McAuley, 2002).

In addition, only one study was identified that focused on results related to the temporal dimension of the stages (Lewis et al., 2006), although the instability of the stages shown in the literature implies the need to address whether, for example, contemplation and action actually last for 6-months. If further findings do not support the 6-month time frame, perhaps an underlying construct of early and late adoption is present, and could be examined with more frequent measures of construct and stage change. As identified for each of the longitudinal studies in this chapter, the bulk have used timelines of 6 months or longer to examine changes in stage and TTM constructs. This is an area of research that should be examined in order to ensure that 6-months (for precontemplation, contemplation, action, and maintenance) is a meaningful time period, and not just an arbitrary marker left over from the development of the model in the smoking literature (Weinstein et al., 1998). Many exercise interventions are 12 weeks in length, due to the physiological changes that are expected to occur during that period. It would be useful to examine behavior change models such as the TTM within these same temporal parameters to maintain a consistency with the typical exercise intervention. 


\section{CHAPTER 3}

\section{METHODS}

\section{Introduction}

The purpose of this study was to test the components of the Transtheoretical Model (TTM) in their ability to explain and predict moderate-and high-intensity exercise at 3 and 6 month follow-ups. Specifically, it was expected that individuals who advanced from an inactive stage to an active stage (adopters) by 6 months would show theoretically based changes in processes of change, decisional balance, and exercise self-efficacy, and that these changes would be predictive of the observed increase in exercise behavior and movement in stage at the 6-month follow-up. It was expected that, according to the model, changes from baseline to 6-months would be better predictors of movement from an inactive to an active stage at 6-months than changes from baseline to 3-months, or changes from 3- to 6-months.

\section{Study Design}

This was a web-based longitudinal study design, with three data collection points (baseline, 3 months, and 6 months). The longitudinal design was selected to provide more explanatory power in identifying discrete stages of change, as well as the mediators for those stages. Cross-sectional designs have been identified as the weakest for 
providing evidence of the existence of discrete stages (Weinstein et al., 1998). Observing how participants change over time allows consistent conclusions to be drawn with respect to time order, as longitudinal examinations afford the opportunity to distinguish whether the proposed changes occur before the actual stage transition.

The sampling plan consisted of a "panel" design, where the initial sample was followed throughout the duration of the study. This allowed us to note behavior patterns over time in our select group of participants, as opposed to re-sampling at each time point (Fink \& Kosecoff, 1998). Participants were working adults in central Ohio, and were randomly selected to receive a recruitment email. The initial sampling frame was an employee list obtained from the human resources departments of three worksites in Central Ohio. Two worksites dropped out of the study before data collection began. Details of this dropout are contained the subsequent section titled "Selection of Participants." All sampling procedures were conducted in accordance with the policies of the Ohio State University Office of Responsible Research Practices Institutional Review Board (IRB). These procedures are described later in this chapter in the sections titled "Selection of Subjects" and "Selection of Worksites."

At baseline, 3-months, and 6-months, participants completed a self-administered web-based questionnaire consisting of the generation of a subject ID, a health screening question (to determine the feasibility of assessing the subject's participation in exercise), demographic items, questions measuring all of the constructs within the TTM (exercise stage of change, processes of change, decisional balance, and exercise self-efficacy), as well as questions related to intention to exercise and exercise participation recall questions. This questionnaire is described in detail in subsequent sections, and a hard 
copy of this questionnaire can be found in Appendices E-P. Table 3.1 shows the timeline of measurement, and the variables that were measured at each point.

It has been recognized that a clear behavioral definition is critically important for behavioral research (Marttila \& Nupponen, 2003; Reed et al., 1997). Classifying respondents into stage of change and assessing concomitant markers of the behavioral criteria will only be valid and reliable if such a behavioral definition is employed (Reed et al., 1997). We used both moderate and vigorous exercise as the behavioral target in this study. Moderate exercise was defined as planned physical activity, which is continuous (20 minutes or more in duration), mildly elevates heart rate, mildly elevates breathing rate, and during which one can hold a conversation while exercising (3-6 METs). MET stands for "metabolic equivalent" and one MET equals $3.5 \mathrm{ml} / \mathrm{kg} / \mathrm{min}$ of oxygen uptake (ACSM, 2000). MET is used as a standardized method for defining energy expenditure in respect to rest. Vigorous exercise was defined as planned physical activity, which is continuous (20 minutes or more in duration), causes the heart to beat rapidly, causes rapid and deep breathing, and during which one can not hold a conversation ( $>6$ METS). In addition to the above definitions, for subjects to meet the exercise criteria they were required to perform moderate and/or vigorous exercise at least three times per week. These definitions were in accordance with the American College of Sports Medicine (ACSM) guidelines for improving or maintaining cardiorespiratory fitness (1998). 


\begin{tabular}{ll}
\hline \multicolumn{1}{c}{ Components } & No. of Items \\
\hline ID Generation & 5 \\
Demographic Items & 10 \\
Injury / Illness Screening & 1 \\
5-choice Stage of Change Algorithm & 1 \\
7-day Moderate Physical Activity Recall & 7 \\
7-day Vigorous Physical Activity Recall & 7 \\
Exercise Intention & 3 \\
Exercise Self-Efficacy & 5 \\
Decisional Balance for Exercise & 16 \\
Processes of Change & 40 \\
5-item Stage of Change Algorithm & 5 \\
\hline
\end{tabular}

Table 3.1 Questionnaire Components at Baseline and Each Follow-Up

\section{Inclusion Criteria}

Participants in the study were required to be working adults, 18-65 years of age, and employed at the designated study sites. The primary analysis was made with participants who were classified in any of the "inactive" TTM stages of change at baseline (Precontemplation, Contemplation, Preparation), however, data from all respondents were collected. Participants were also required to have access to the internet, 
as this was a web-based survey. Finally, the participants were required to be free from any diseases or injuries that would prevent them from participating in regular exercise throughout the study, such as orthopedic injuries or chronic diseases.

\section{Selection of Worksites}

To recruit worksites for participation in the study, worksite wellness directors and human resource administrators were contacted. Three worksites were interested in the research and met with the researcher. At this meeting, the basic purpose of the study was discussed, and the design and requirements of both the worksite and the employees were outlined. After initial approval was gained, further contact was arranged to deliver an invitation letter and overview of the project, which was used in part to assist in obtaining a letter of approval from the worksite for the Ohio State University IRB, which can be found in Appendix B. The invitation and overview, as well as the letters of approval can be found in Appendices A and C, respectively. The invitation letter included a purpose statement, information regarding the benefits and protocol for the study, and a link to a web sample of the survey (this was a separate website from the actual survey). The overview included bulleted details about the study protocol, as well as the commitment required of participants and benefits to the worksite. Three worksites returned the letter of approval per IRB guidelines, and were considered to be participating worksites at the start of participant selection.

\section{Selection of Participants}

One-thousand six-hundred randomly selected employees were targeted at the outset of the study. Employees were initially divided among the three central Ohio worksites that provided approval letters, and the targeted sample size at each worksite was proportional 
to the total number of employees at that worksite. The three worksites consisted of a large university, a large utilities company, and a medium-sized Medicaid case management company. A final sample size of 168 working adults over the three worksites and was needed to serve as participants for the study. This number was determined using power calculations based on previous research on self-efficacy as a predictor for inactive to active stage transition (Plotnikoff et al., 2001). Self-efficacy was chosen because it is the variable with the most consistently established positive relationship with exercise behavior change. The change in self-efficacy reported in the Plotnikoff et al (2001) study (mean change score in self-efficacy between the preparation and action stages of 1.42) was used to calculate sample size because their research design most closely resembled ours. The sample size formula was as follows:

$$
\begin{aligned}
& \mathrm{n}=\left[\sigma^{\wedge} 2(\mathrm{Z} 1-\alpha / 2+\mathrm{Z} 1-\beta)^{\wedge} 2\right] /(\mu 1-\mu 0)^{\wedge} 2 \\
& \text { where } \\
& \alpha^{\wedge} 2=\text { population variance } \\
& \mathrm{Z} 1-\alpha=\text { the } \mathrm{z} \text { value for a given confidence level }(1-\alpha) \\
& \mathrm{Z} 1-\beta=\text { the } \mathrm{z} \text { value for a given power, and } \\
& \mu 1-\mu 0=\text { the difference in population means }
\end{aligned}
$$

The result was as follows:

$$
\begin{aligned}
& \mathrm{n}=\left[\sigma^{\wedge} 2(\mathrm{Z} 1-\alpha / 2+\mathrm{Z} 1-\beta)^{\wedge} 2\right] /(\mu 1-\mu 0)^{\wedge} 2 \\
& \mathrm{n}=[31.36(10.82)] /(1.42)^{2} \\
& \mathrm{n}=168
\end{aligned}
$$

For these calculations power was set to .8 and $\alpha$ to .05 . The number of initial contacts was determined using justifications based primarily on the work of Plotnikoff et 
al (2001), who examined the TTM among Canadian adults using a telephone sample over a 12-month follow up. The authors found that $44 \%$ of baseline respondents remained in the study after 12 months, and 128 out of 374 respondents $(\sim 34 \%)$ who were inactive at baseline (as measured by stage of change) had progressed to an active stage by the end of the study (Plotnikoff et al., 2001). For our study, the necessary sample size of 168 that was calculated in the previous section represented the $34 \%$ of retained participants who would make the transition from inactive to active by the end of the study. Therefore, we needed $494(168 / .34)$ participants to remain in the study throughout the 6 months. Based on a retention rate of $44 \%$, this would require $1122(494 / .44)$ respondents to the initial mailing. Assuming a 70\% response rate based on methods described by Dillman (2000), we needed an initial mailing of approximately 1600 surveys (1122/.70) to obtain the needed sample size.

Initially, the subjects were to be divided proportionally by worksite. This division is outlined in Table 3.2. Though three worksites returned letters of approval (Appendix C), only one (the large university) was immediately cooperative in providing the sampling frame. For each of the two worksites that did not immediately provide the employee list, three subsequent contacts were made via email and two via phone to attempt to begin the sampling process. Though the initial reply to these contacts indicated continued interest in participation, final phone contacts during the last week of October, 2006 were not returned. To finish the study in a timely manner (per the completed IRB application), and to avoid confounding the data with seasonal differences between responding worksites, these two worksites were considered non-compliant and were dropped from the study. 
When the two non-compliant worksites became non-responsive to initial contacts, a decision was made to sample the necessary 1600 employees from the compliant (and largest) worksite. A selection letter was sent out to the 1600 randomly selected employees via email, and a paper copy was delivered via internal (campus) mail to each employee's mailbox. The letter included an explanation of the purpose of the study, an advance 'thank you' for participation, the web address for the online survey, and a username/password for entering the survey site. Those who read the letter from their email were able to link directly to the web survey immediately. A sample of the invitation letter can be found in Appendix D.

\begin{tabular}{llll}
\hline \multicolumn{1}{c}{ Worksite } & \# of Employees & \% of Total & $\begin{array}{c}\text { Target Sample } \\
\text { Size }\end{array}$ \\
\hline University & 12000 & 83.9 & 1342 \\
$\begin{array}{l}\text { Medicaid Case } \\
\text { Management Co. }\end{array}$ & 300 & 2.1 & 34 \\
$\begin{array}{l}\text { Utilities Co. } \\
\text { Totals }\end{array}$ & 14,3000 & 14.0 & 224 \\
\hline
\end{tabular}

Table 3.2: Initial Sampling Scheme

To reduce non-response, several repeat contacts were performed, as suggested by Dillman (2000). Respondents had 10 days to complete the survey after the first email / office mail contact. If the survey was not completed, a second email and office mailing were sent, emphasizing a desire for a high response rate and the importance of the study. If no response was received after 10 additional days, a third email was sent, again highlighting the importance of response and of the study, and emphasizing that it was not 
too late to participate. After 10 more days, the employee was counted as a nonrespondent. Dillman (2000) has reported response rates greater than $70 \%$ using these methods. This approach was repeated for each of the subsequent follow-ups. All invitation and reminder letters can be found in Appendix D. For study drop-outs, available demographic data was compared with respondent data to evaluate whether the responding sample was representative of the population. These data can be found in the results section of this paper.

\section{Subject Participation}

When arriving at the website, participants were asked to enter the username and password that was supplied in the initial letter. This minimized the risk of someone who was not invited to participate in the study browsing the web entering data that would corrupt the study results. A username (websurvey) and password (survey) that were both easy to remember and non-reactive were chosen for this purpose. Initially, "exercisesurvey" and "exercise" were considered for the username and password, but it was decided that the inclusion of the word "exercise" could influence the respondents to provide biased answers.

After successfully entering this information, participants were welcomed to the first page of the Worksite Exercise Behavior Study (WEBS), and asked to enter the email address at which they received their invitation to participate. This was included so that participants could be identified and marked off the list for future contact. Data from this

page were stored in a different Excel file from the main survey. Consequently, it was not possible to link the email address with the completed survey data, which allowed for respondents' anonymity to be maintained. 
This initial page also served as a reminder to the participant that his or her information would be treated with "absolute confidentiality" and that "no responses will be individually identifiable or shared with your employer." Participants were further reminded that they could choose not to participate or withdraw from the study at any time without penalty. A copy of the survey, which shows the wording for this and all subsequent pages can be found in Appendices E through P.

Once the email address was submitted, participants were taken to a page containing a reminder that none of the individual responses will be shared with employers. Participants were given the researchers' contact information, as well as contact information for the University Office of Responsible Research Practices.

At the next page, participants were asked to create a unique 5-character ID by entering the following data:

- $\quad$ First letter of the company for which they work (e.g., Corporate Incorporated $=\mathrm{C})$

- $\quad$ The first letter of the city in which they were born (e.g., Columbus=C)

- $\quad$ The last digit of the zip code in which they live (e.g., 43229=9)

- $\quad$ The number of brothers they have

- $\quad$ The third letter of the month in which they were born (e.g., March=R)

These categories were chosen because they tend to be fairly static, yet they allow for participant anonymity to be maintained. This code served as the participant's ID, and was used to match follow-up data with baseline data for statistical analysis. All data in the main file was identified using only this unique ID. Additionally, IP addresses from which the respondent completed the survey were not stored. Data were stored on the 
P.I.'s computer, the co-investigator's computer, and on the School of PAES server, to which only the P.I., the School of PAES Technology Specialist (Laura Fathauer), and the co-investigator (Christopher Fink) have access. For security reasons, the P.I. and the coconvestigator's computers are password protected.

Measures-Web Survey Software

To implement the questionnaires in a web-based format, Select Survey ASP by ClassApps $\left(\mathcal{C}\right.$ was used. The survey was initially designed using Microsoft FrontPage ${ }^{\circledR}$ but there were problems in communication between FrontPage and the University server that caused certain users to not have access to the survey.

Select Survey ASP allowed the questions to be presented with very few issues related to accessibility, and eliminated concerns regarding the appearance of the survey being dependent upon the browser that the respondent utilized. Additionally, features like a standard progress bar, and customizable colors and fonts gave the survey a professional appearance which was not evident with the FrontPage ${ }^{\circledR}$-based version. Select Survey ASP used a comma delimited format to store the data, which allowed the researcher to download data in a format that was easily managed by Microsoft Excel ${ }^{\circledR}$ and SPSS.

There were a few limitations of using this software package to design the study. First, questions that required Likert-scale responses were difficult to design without a "category" for the horizontally numbered scale. To combat this, a non-reactive symbol (period) was entered as a title, which allowed the Likert scale to be viewed horizontally, as seen in Figure 3.1 below. 


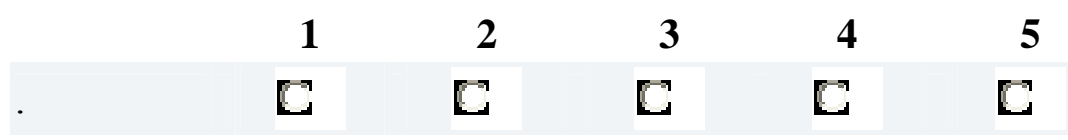

Figure 3.1 - Likert Scale with Period at Left

Additionally, the use of FrontPage ${ }^{\circledR}$ would have allowed the survey data and email addresses to be immediately downloaded into separate files upon submission by the respondent in order to maintain respondent anonymity. SelectSurvey ASP did not allow this to happen, but upon manual download and a check for incomplete or erroneous data, email addresses and data were separated into separate files for storage, so no responses were able to be linked to the individual respondent.

Measures - Questionnaire

The components of the questionnaire were valid and reliable instruments that have been used in previous exercise behavior research. These components were combined into one web-based instrument, which was pilot-tested with a group of 21 working adults in central Ohio for feedback on readability, clarity, and time-feasibility. Twenty subjects is the established minimum necessary for pretesting an instrument (Fowler, 2002; McMillan \& Schumacher, 1997).

Those responding to the pilot test reported taking an average of 17.3 minutes to complete the survey, and brought to the attention of the researcher several misspellings within the survey, and issues with navigating backward to previous pages in order to alter their answers. There were also three requests for clarification about the wording of 
questions, however, there were not multiple requests for clarification of any one question. Additionally, 17 pilot test respondents reported using Microsoft Internet Explorer® to complete the survey, one reported using Netscape $\mathcal{C}$, and two used Mozilla Firefox ${ }^{\mathrm{TM}}$. All were able to view and complete the survey without problems, aside from the previously mentioned issue with navigating backwards.

A web format was chosen to increase the likelihood of response, as participants had web access through the worksite, and preliminary approval was given to allow participants to take the time to complete the questionnaire at work. In addition, the majority of the coding of the questions and responses was done as a part of the survey design, so data management and analysis following the study was less cumbersome, and the costs associated with mailings, telephone contacts, and data entry were lowered (Dillman, 2000).

Select Survey ASP Advanced by Class Apps (C) uses a familiar Windows-based "point-and-click" format, which allows the participant to navigate easily through the survey. The survey contained very few graphics, and had no advanced animation or audio/video components. If the technology used in designing and displaying the survey would have been too advanced and the participants would have had have out-of-date technology, there was an increased chance that the participants would have been unable to respond due to slow download times and / or problems with viewing the survey. Additionally, any participants who had elected to complete the survey at a location outside of work could have had a lower connection speed, which would have increased the amount of time required to complete a graphics-heavy survey. 
There were several other decisions made regarding the survey design. First, most data were recorded using radio buttons. Radio buttons allowed the respondent to choose only one option, which was appropriate for most of the items in this survey. Exceptions to this are described in the appropriate sections. Also, Select Survey ASP provides a standardized view of the survey that minimizes the chance that survey text would not be visible to participants with different monitor and text size settings on their computer systems. Progress bars were also included in the body of the survey. These bars helped the respondents to visualize how much of the survey remained, which was included to reduce the chance for frustration about the length of the survey. Finally, all data were contained within one "submission," with columns for each data point. This reduced the chance for problems when submitting data, as there were fewer opportunities for missed submissions by the respondents. It also allowed for respondents to verify their answers if needed. Respondents were permitted to select the "back" button to view and change previous answers before the final submission of the form. Following is a section-bysection discussion of the survey and individual questionnaires.

Demographics. Demographic information was collected at the beginning of the survey. This information included participant sex, age, ethnicity, marital status, selfreported height and weight, education, job status, and job title. The sex, age, race, martial status, height, and weight portions of the survey were taken from the 2004 Behavioral Risk Factor Surveillance System questionnaire (CDC, 2004). A few of these items differed from the standard radio button choices found in other sections. The respondent was asked to enter his or her age in a textbox in this section. This textbox had a limit of two characters, as all respondents were working, and under 100 years of age. Also, 
respondents were allowed to choose more than one item for their race, as many people identify as a combination of races. A choice of don't know / prefer not to answer was also provided in this section, which allowed the respondent to choose not to answer this item. Respondents were required to manually enter their height in feet and inches, as well. These boxes were limited to one and two characters, respectively. Similarly, respondents entered their weight in a textbox limited to three characters. The choice of 'prefer not to answer' was also included for all items with forced choices in this section. A sample of this portion of the instrument can be found in Appendix F.

Check for Injury or Illness. The following one-question check for injuries or illness was included in the instrument: "Do you have any injuries or illnesses that will prevent you from exercising over the next 6 months?" If participants selected 'Yes,' they were taken to the end of the instrument, thanked for participation, and invited to submit their data. These participants were then tagged as having completed the study, as injury was identified as an exclusion criterion. These respondents were not included in the primary data analysis, though they were used in a descriptive comparison with those who completed the study. If 'No' was selected, the participant was taken to the next section of the survey. A sample of this portion of the instrument can be found in Appendix G.

Exercise Stage of Change. Exercise stage of change was assessed with two different algorithms to check for consistency of measurement. Stage of change was first assessed with the Long, Vigorous 5 Choice exercise stage of change algorithm described by Reed et al. (1997). This algorithm consists of a long definition for exercise and five choices for stage of change. Exercise is defined as follows: "Activities such as brisk walking, jogging, swimming, aerobic dancing, biking, rowing, weight lifting, etc. 
Activities that are primarily sedentary such as bowling, or playing golf with a cart, would not be considered exercise." Exercise stage of change was assessed by first asking the following question: "Do you exercise regularly, that is, 3 or more times each week for at least 20 minutes each time?" This was followed by 5 choices that served to classify the participant into one of the stages of change. Participants were able to select a radio button under the statement that corresponded with their current exercise stage of change.

Following are the choices that were given, with the associated stage in parentheses: "Yes, and I have been for more than 6 months (Maintenance); Yes, and I have been for less than 6 months (Action); No, but I am planning to start in the next 30 days (Preparation); No, but I am planning to start in the next 6 months (Contemplation); and No, and I don't have plans to start in the next 6 months (Precontemplation).” A sample of this algorithm can be found in Appendix $\mathrm{H}$.

An examination by Reed et al. (1997) compared several exercise stage of change algorithms, and found that this version produced the highest degree of external validity. This was identified by using MANOVA to evaluate patterns of 'pros' and 'cons,' as well as weekly hours of exercise by exercise stage of change. The classical pattern of increasing pros across stages, with a decrease from action to maintenance, and a decrease in cons across stages was most clearly demonstrated with the Long, Vigorous, 5 Choice algorithm, as was a linear increase in weekly hours of exercise, rising above the criteria level at the action stage.

Exercise stage of change was also measured with the Long Vigorous True/False algorithm as described by Reed et al. (1997). This algorithm consists of the same definition for regular exercise as the first algorithm, and five different statements, to 
which the respondents must answer 'yes' or 'no.' These statements are as follows: (1) I currently exercise; (2) I intend to exercise in the next 6 months; (3) I currently exercise regularly; (4) I have exercised regularly for the past 6 months, (5) I have exercised regularly in the past for at least 3 months." The following are the stages and their respective answers to the above statements:

- $\quad$ Precontemplation: No on questions 1-4

- $\quad$ Contemplation: No on questions 1, 3, and 4 and Yes on question 2.

- $\quad$ Preparation: No on questions 3 and 4, and Yes on questions 1 and 2

- $\quad$ Action: No on question 4, and Yes on questions 1-3

- $\quad$ Maintenance: Yes on questions 1-4

This algorithm was chosen because the Reed et al. (1997) examination found that there was little variation (no more than $6 \%$ ) in the proportion of their sample who staged themselves in each stage between the True/False and the 5-choice formats. The primary difference was in external validation, where the classical pattern of increasing pros across stages was only disturbed at the preparation stage, then returned to a smooth rise through the action stage. Other algorithms produced more differences in patterns of pros/cons and confidence as compared to the 5-choice algorithm. Question 5 was included to maintain the consistency of the algorithm with the purpose of the study, that is, to examine the factors that predict the change in exercise behavior at 3 months as well as at 6 months post-baseline. An example of this algorithm can be found in Appendix O.

Exercise Behavior. Exercise behavior was measured using an adapted version of the Self-Report 7-day Physical Activity Recall (Petosa, 1995). This is a 2-part instrument that measures minutes of participation in both moderate and vigorous physical activity. It 
is based on the 7-day physical activity recall designed by Blair et al.(1985), which was found to be correlated to maximal aerobic capacity $(\mathrm{r}=.33)$. Petosa modified the instrument to include more information about frequency, duration, and activity type. This modified version had test re-test reliabilities ranging from $\mathrm{r}=.52$ for free living activity to $\mathrm{r}=.72$ for supervised activity, and correlated with 7-day diaries of physical activity $(\mathrm{r}=.72)$.

In this instrument, moderate exercise is defined as "planned physical activity which is continuous, mildly elevates heart rate, mildly elevates breathing rate, and allows the participant to hold a conversation while exercising." Examples of moderate exercise are given, and include weight lifting, aerobic dance, swimming (no laps), low-impact exercise/strength classes, brisk walking, cycling less than 12 miles per hour, among others. Vigorous exercise is defined as "planned physical activity which is continuous, in which the heart beats rapidly, breathing is rapid and deep, and does not allow conversation while exercising." Examples of vigorous exercise are given, and include running or jogging, high-intensity aerobics classes, competitive field sports (soccer), competitive full-court basketball, among others. Examples were taken from the Compendium of Physical Activities (CDC, 2004) and the original survey (Petosa, 1995).

Respondents were asked to indicate on which days they participated in each type (moderate and vigorous) of physical activity, and for how many minutes. This instrument was modified for the web-format by staring with "yesterday" and proceeding to "two days ago," "three days ago," and so on. Respondents were also asked to identify which day (e.g., Monday, Tuesday, etc.) corresponded with each response. Respondents were asked "Did you engage in any moderate exercise yesterday?" to which they 
responded "Yes" or "No" by clicking on a radio button. Then, respondents entered the number of minutes of exercise that were completed on that day in a textbox. Finally, to select their activity(ies), checkboxes were available for the aforementioned example exercises, as well as a box for "other, please specify" where the respondent could enter activities that weren't included in the example list. This instrument was selected because it tracks well with the behavioral definition used in this study, as vigorous physical activity is defined as planned, and lasting more than 20 minutes. A sample of this instrument can be found in Appendices I and J.

Processes of Change. Processes of change were measured using the Processes of Change instrument developed by Marcus, et al. (1992), and revised by Norman (1998). This instrument consists of 40 items designed to assess the 10 processes of change proposed in the TTM. Internal consistency measures (coefficient alpha reliabilities) for these processes ranged from $\alpha=.64$ to $\alpha=.86$. Concerns over sample size for this study in respect to number of predictor variables led to analyzing the processes as two higher order processes of change (behavioral and cognitive) consisting of 20 items each. This two factor iteration of the processes of change was validated for use in exercise research by Marcus et al. (1992)

The directions for this instrument are: "The following experiences can affect the exercise habits of some people. Think of any similar experiences you may be currently having or have had during the past month. Then, rate how frequently the event occurs. Using the rating system shown below, please click the button below the number than best describes your answer." Respondents were then asked to score how frequently each statement occurs on a 1-5 Likert scale, ranging from 1 (never), to 5 (repeatedly). 
Respondents were asked to then choose a radio button that corresponds to the appropriate Likert rating for that item. A sample of this instrument can be found in Appendix K.

Decisional Balance. Decisional balance (DB) was assessed using the Decisional Balance questionnaire from Marcus, Rakowski, and Rossi (1992). This is a 16 item instrument with 10 pro-exercise items and 6 con-exercise items. The instrument assesses the respondent's perceived positive and negative aspects of exercise (Marcus, Rakowski et al., 1992). For the entire sample, at each measurement point (baseline, 3-months, and 6-months) each item response is converted to a t-score, and a mean t-score is calculated for both the 'pros' and 'cons' scale. Decisional balance (DB) is then represented as the score on the "Cons" scale subtracted from the score on the "Pros" scale (DB=Pros-Cons).

Respondents were asked to: "Please rate how important each of these statements is in your decision whether or not to exercise. In each case, think about how you feel right now, not how you have felt in the past or would like to feel. Using the rating system shown below, please click the circle below each statement that best describes your answer." Respondents were then asked to rate the importance of each statement on a 5point Likert scale, where 1 is "not at all important in my decision whether or not to exercise," 3 is "moderately important in my decision whether or not to exercise," and 5 is "extremely important in my decision whether or not to exercise." This instrument has demonstrated reliability in exercise behavior research, with internal consistency measures (coefficient-alpha reliabilities) of .79 and .95 for cons and pros, respectively (Marcus, Rakowski et al., 1992). Respondents were asked to choose a radio button that corresponded to the appropriate Likert rating for each item. A sample of this instrument can be found in Appendix L. 
Self-Efficacy. Self-efficacy was measured using the Exercise Confidence scale (Marcus \& Simkin, 1993). This is a 5-item instrument that uses a 5 point Likert-type scale (1, "not at all confident," to 5, "extremely confident") to assess the confidence of the respondent that they could exercise in certain "barrier" situations, including poor weather, fatigue, lack of time, poor mental state, and when on vacation. Instructions for this instrument are: "Please click the appropriate button to rate how confident you are that you could exercise in each of the following situations." T-scores were then calculated for each item, and the results were used to compute an average t-score for each respondent. Internal consistency for this measure was reported as .82 (Marcus \& Simkin, 1993). Respondents were asked to choose a radio button that corresponds with the appropriate Likert rating for each item. A sample of this instrument can be found in Appendix M.

Intention. Intention was measured using a 3-item, 4-point Likert-type scale (1, "not at all true," to 4, "absolutely true") that was adapted from a measure initially developed by Lippke, Ziegelmann, and Schwarzer (2004). The intention scale's exercise definition of " 2 or more days per week, 20 minutes per session" was changed to "three or more days per week, 20 minutes per session" to maintain a consistent behavioral criteria for this study. Respondents were asked to choose a radio button that corresponded with the appropriate Likert rating for each item. A mean intention score was then calculated from the three items. Chronbach's alpha for the original scale ranged from .53-.74, indicating moderate reliability of the measure (Lippke et al., 2004). A sample of this instrument can be found in Appendix N.

\section{Data Management}


Survey data were downloaded from the survey website onto the co-investigator's computer at 10 day intervals following the initial invitation to participate, at the same time that reminder prompts were sent to those who had not yet completed the survey. Data were saved in a comma-delimited format for cleaning and sorting in Microsoft Excel $\odot$, and filenames were used that indicated the date of download. At each download, submissions were reviewed for errors or incomplete submissions, and an email was sent to these respondents requesting that they re-submit their responses. An example of this email can be found in Appendix D. After this check, email addresses were removed from the data files and stored separately. All data were subsequently summed, coded, and transferred to t-scores as needed within Microsoft Excel®. Data were then imported into SPSS for analysis.

Data Analysis

Data were identified only by the 5-characted ID that each participant generated at the outset of the survey. Data were analyzed using the Statistical Package for the Social Sciences, version 15.0 (SPSS, Inc., Chicago, IL, 2006). Descriptive statistics are available for all variables in tables 4.1 and 4.2 including the mean and standard deviation for continuous variables and frequency distributions for nominal variables. All inferential analyses were conducted at an a priori significance level of $\alpha=.05$.

The dependent variable in this study was adoption of an active stage of change between baseline and 6 months, as measured by the Long, Vigorous 5-choice stage of change instrument (Reed et al., 1997). Participants who moved from an inactive stage (Precontemplation, Contemplation, Preparation) to an active stage (Action, Maintenance) by the 6-month measurement were coded as "inactive-active," or I-A, while participants 
who were inactive at baseline and did not move from an inactive to an active stage were coded as "stable-inactive," or S-I. Descriptive data for these groups are reported in the results section of this paper.

The independent variables for this study were baseline to 3-month, 3- to 6-month, and baseline to 6-month changes in decisional balance for exercise, self-efficacy for exercise, behavioral processes of change, and cognitive processes of change. Since the dependent variable is dichotomous (I-A vs. S-I), logistic regression was employed in predicting stage transition.

One of the assumptions of logistic regression is that the predictor variable must be linear in the logit. An observed lack of such linearity in each of the predictors in this study resulted in each of the predictors being transformed to dichotomous scores based on changes observed in the model-development literature, according to the approach described elsewhere by Hosmer and Lemeshow (2000). Specifically, the criteria for the cut-points in decisional balance scores were based on the original hypothesis by Prochaska and colleagues that proposed that advances from preparation to action (inactive to active) are predicted by a $1.0 \mathrm{SD}$ increase in pros and a $.50 \mathrm{SD}$ decrease in cons (Prochaska et al., 1994). The criterion values for self-efficacy change (>.50 SD change in score), and change in behavioral ( $>.48$ SD change in score) and cognitive $(>.32$ SD change in score) processes of change were taken from landmark studies applying the TTM to exercise (Marcus et al., 1998; Marcus, Rossi et al., 1992).

Change scores have come under some criticism as being unreliable for use in regression analyses because of the greater tendency of extreme scores to regress toward the mean. This was minimized in our study because the independent variables were 
dichotomized in a manner consistent with foundational research in the TTM and exercise, and a rigid criterion was set for increase in each construct.

Univariate logistic regression analyses were then conducted for baseline to 3month, 3- to 6-month, and baseline to 6-month change scores in each independent variable as predictors for membership in the S-I or I-A groups. Any predictors with $p$ values less than .25 were then entered into the multivariate logistic regression model for each transition period (Hosmer \& Lemeshow, 2000). Backward elimination of the independent variables was performed until the model likelihood ratio reached a $p$-value less-than or equal to .05. The strength of the predictors was examined using the Wald statistic, and overall model fit was assessed using the Hosmer-Lemeshow goodness-of-fit test. Odds ratios (OR) were examined for further indication of the strength of prediction of each independent variable. 


\section{CHAPTER 4}

\section{RESULTS AND DISCUSSION}

Introduction

Physical inactivity has been well-documented as an independent risk factor for a variety of chronic diseases, including coronary heart disease (CHD), type II diabetes, hypertension, and colon cancer (U.S. Department of Health and Human Services, 2000). An inactive lifestyle has been shown to contribute to mental health issues, osteoporosis, and obesity (U.S. Department of Health and Human Services, 1996). Direct medical costs from physical inactivity have also risen from \$29 billion in 1987 to more than $\$ 76$ billion in 2000 (Centers for Disease Control and Prevention, 2000), and diseases related to inactivity contribute to greater absenteeism and lower productivity (Bull, Gillette, Glasgow, \& Estabrooks, 2003). Regular physical activity is protective against chronic disease, but physical activity rates are disappointing, with over $60 \%$ of U.S. adults engaging in insufficient levels of physical activity for garnering health benefits (Centers for Disease Control and Prevention, 2000). Additionally, most people who begin a regular exercise program drop out within 6 months, despite the efforts of health and exercise professionals (Dishman, 1988). 
Theories of behavior change have been used to analyze these disappointing rates of physical activity and poor adherence, as well as to design physical activity interventions through targeting constructs proposed by the theory to mediate behavior change. However, interventions are often based on a premature use of theory, that is, constructs strongly correlated with the target outcome, in this case exercise, are assumed to have causal relationships even before such relationships have been verified through experimental studies or even demonstrated to track longitudinally (Dishman, 2001). In addition, theories developed for other health behaviors, such as smoking and nutritional choices, have been adapted for use with exercise (Ajzen, 1991; Fishbein, 1967;

Prochaska \& DiClemente, 1983), although the dynamics of physical activity are quite different from other health behaviors. For example, the typical objective for physical activity interventions is adoption of the behavior, while the goal for smoking is cessation. Other dynamics of the health behavior can also vary, such as frequency of change and physiological characteristics (e.g. addiction to nicotine versus physiological activation with exercise).

The transtheoretical model of behavior change (TTM) (Prochaska \& DiClemente, 1983) was developed from a naturalistic inquiry into the smoking cessation process, but has been applied to exercise since the early 1990s (e.g. Marcus, Selby et al., 1992). Over the past 15 years, the TTM has been used and studied extensively in physical activity behavior interventions and research (Marshall \& Biddle, 2001). Several core constructs were developed, including five stages of change (Precontemplation, Contemplation, Preparation, Action, and Maintenance), which are defined temporally both by the health behavior and an individual's motivational readiness to change, generally in 6-month 
increments (Prochaska \& DiClemente, 1983; Prochaska et al., 2002). The key tenet of the TTM is that behavior change is a dynamic process that involves motivational readiness, as well as a temporal dimension, and that progress through the stages of change is related to changes in three major factors (processes of change, decisional balance, self-efficacy) (Marshall \& Biddle, 2001; Prochaska et al., 2002). Processes of change are strategies used to foster the behavior change. Relevant information generated by someone's own actions or experiences are considered cognitive or experiential processes, while for behavioral processes, information is generated by environmental events and behaviors. The original hypothesis (Marcus \& Simkin, 1993; Prochaska \& DiClemente, 1983; Prochaska \& Velicer, 1997b) stated that the cognitive processes of change are more important in moving through the earlier stages of change, while the behavioral processes become more important later in the behavior change process.

Exercise self-efficacy is proposed to increase across the progression through the stages of change, while pros of exercise increase and cons of exercise decrease, with the "cross-over" point (the point at which the pros overtake the cons) coming at the transition from the preparation to the action stage (Marcus, Rakowski et al., 1992; Marshall \& Biddle, 2001; Plotnikoff et al., 2001; Prochaska et al., 2002). Self-efficacy has a strong, consistent relationship with stage advancement, and it has been postulated that individuals may be able to be differentiated by stage simply on ratings of exercise or physical activity self-efficacy (Marshall \& Biddle, 2001).

Finally, though one of the strengths of the TTM is its inclusion of a temporal dimension for classifying the change process, it is possible that the 6-month increments used in the original model are specific to smoking, and may be different based on the 
health behavior in question. For example, the transition from the action to the maintenance stage is primarily a function of remaining active for at least six months. Though this time frame is consistent based on the literature related to drop-out (Dishman, 1988), many exercise interventions are designed to be 12 weeks in duration, which is a time period associated with physiological adaptations to regular exercise (Kearney et al., 1976; Pollock et al., 1998). Changes in physiological variables (e.g., increased aerobic capacity and endurance, decreased fatigue) are potentially reinforcing outcomes, and therefore could factor into an individual's decision to remain physically active. The hypothesized changes in processes of change, self-efficacy, and decisional balance for exercise may occur in conjunction with training adaptations, which can occur earlier than the 6-month cutoff.

Cross-sectional examinations of theory have been cited as a weak test of the constructs and processes involved with the TTM (Weinstein et al., 1998). However, most evidence for the usefulness of the TTM, including the initial adaptation of the TTM to exercise, has been primarily based on studies using a cross-sectional design, rather than costly and time consuming longitudinal or experimental designs. As such, they can only describe the characteristics of individuals falling into the varying stages of change (Buckworth \& Wallace, 2002; Cardinal, 1997a; Marcus, Banspach et al., 1992a; Marcus, Rakowski et al., 1992; Marcus, Rossi et al., 1992; Marcus, Selby et al., 1992; Spencer, Adams, Malone, Roy, \& Yost, 2006; Sutton, 2000).

In recent years, the number of longitudinal tests of the TTM with exercise has increased, though few have incorporated all of the TTM constructs. Plotnikoff et al. (2001) examined the complete model in a population sample over a one year follow-up 
with no behavioral intervention, to mimic the method used in the development of the TTM in the smoking literature. The authors reported support for approximately $45 \%$ of 40 possible predictions, which were based on the original TTM model. Other longitudinal studies have found baseline stage (Armstrong et al., 1993; Cardinal, 1997b), self-efficacy (Higgins \& Oldenburg, 2003; Maddison \& Prapavessis, 2006; Rhodes \& Plotnikoff, 2006), and behavioral processes of change (Rhodes \& Plotnikoff, 2006) to be significant predictors of stage transition. Only one study has examined constructs theorized to predict changes in stage over a shorter time period than the hypothesized 6months. Lewis et al. (2006) found that positive changes in behavioral processes of change and self-efficacy from baseline to 3 months were significantly related to positive change in minutes of physical activity when controlling for baseline levels of physical activity. These results further call into question the rigidity of the 6-month time period as hypothesized in the TTM.

A further examination of the 6-month time course for exercise may yield insight into the dynamics of behavior change between initial stage classification and the 6-month benchmark. Therefore, the purpose of this study was to test 3-and 6-month changes in the components of the TTM (processes of change, self-efficacy, decisional balance) in predicting adoption of an active stage of change from baseline to 6-months post-baseline assessment. It was hypothesized that predictions based on baseline to 6-month changes in TTM constructs would more closely resemble the full TTM model than baseline to 3month changes and 3- to 6-month changes. 


\section{Study Design}

This was a web-based longitudinal panel study design, with three data collection points (baseline, 3 months, and 6 months). The sample was drawn from an employee list obtained from the Human Resources Department of a large Midwestern university. Participants in the study were required to be working adults, 18-65 years of age, employed at the study site, have access to the internet, and be free from any diseases or injuries that would prevent them from participating in regular exercise throughout the study, such as orthopedic injuries or chronic diseases. One-thousand six-hundred randomly selected employees were targeted at the outset of the study based on a priori power of .8 and $\alpha$ of .05 . To reduce non-response, several repeat contacts were performed (Dillman, 2000).

At baseline, 3-months, and 6-months, participants completed a self-administered web-based questionnaire consisting of the generation of a subject ID, a health screening question (to determine the feasibility of assessing the subject's participation in exercise), demographic items, questions measuring all of the constructs within the TTM (exercise stage of change, processes of change, decisional balance, and exercise self-efficacy), and exercise participation recall questions.

Moderate- or high-intensity exercise was chosen, rather than the more global measure of physical activity, because of evidence indicating that stage of change measures can be less reliable and valid when examining lower-intensity activities (Leslie et al., 2003; Schumann et al., 2003). Moderate exercise was defined as "planned physical activity, which is continuous (20 minutes or more in duration), mildly elevates heart rate, mildly elevates breathing rate, and during which one can hold a conversation while 
exercising (3-6 METs)." Vigorous exercise was defined as "planned physical activity, which is continuous (20 minutes or more in duration), causes the heart to beat rapidly, causes rapid and deep breathing, and during which one can not hold a conversation $(>6$ METS)." In addition to the above definitions, for subjects to meet the exercise criteria they were required to perform the moderate or vigorous exercise at least three times per week.

\section{Questionnaire}

Participants were asked to create a unique 5-character ID, followed by questions about demographic information, specifically gender, age, race, martial status, height, and weight, which were taken from the 2004 BRFSS questionnaire (CDC, 2004), in addition to education, job status, and job title. A one-question check for injuries or illness was included in the instrument, as well. Exercise stage of change was assessed with Long, Vigorous 5 Choice exercise stage of change algorithm described by Reed et al. (1997). Exercise behavior was measured using an adapted version of the Self-Report 7-day Physical Activity Recall (Petosa, 1995). This is a 2-part instrument that measures minutes of participation in both moderate and vigorous physical activity, and is based on the 7day physical activity recall designed by Blair et al.(1985). Processes of change were measured using the Processes of Change instrument developed by Marcus, et al. (1992), and revised by Norman (1998). This instrument consists of 40 items designed to assess the 10 processes of change proposed in the TTM. Decisional balance (DB) was assessed using the Decisional Balance questionnaire from Marcus, Rakowski, and Rossi (1992). This is a 16 item instrument with 10 pro-exercise items and 6 con-exercise items. Selfefficacy was measured using the Exercise Confidence scale (Marcus \& Simkin, 1993). 
This is a 5 -item instrument that uses a 5 point Likert-type scale (1, "not at all confident," to 5, "extremely confident") to assess the confidence of the respondent that they could exercise in certain "barrier" situations, such as poor weather and lack of time.

\section{Data Management and Analysis}

Survey data were downloaded at 10 day intervals following the initial invitation to participate, at the same time that reminder prompts were sent to those who had not yet completed the survey. Data were identified only by the 5-characted ID that each participant generated at the outset of the survey. Data were analyzed using the Statistical Package for the Social Sciences, version 15.0 (SPSS, Inc., Chicago, IL, 2006). All inferential analyses were conducted at an a priori significance level of $\alpha=.05$.

The dependent variable in this study was adoption of an active exercise stage between baseline and 6 months, as measured by the stage of change instrument. To preserve power, participants who moved from an inactive stage (Precontemplation, Contemplation, Preparation) to an active stage (Action, Maintenance) by the 6-month measurement were coded as Inactive-Active adopters, or "I-A," while participants who were inactive at baseline and did not move from an inactive to an active stage were coded as Stable-Inactive, or "S-I." Descriptive data for all groups are reported in the results section of this paper.

The independent variables for this study were baseline to 3-month, 3- to 6-month, and baseline to 6-month changes in self-efficacy for exercise, behavioral processes of change, cognitive processes of change, and decisional balance (pros and cons) for exercise. Since the dependent variable is dichotomous (I-A vs. S-I), logistic regression was employed in predicting stage transition. 
One of the assumptions of logistic regression is that the predictor variable must be linear in the logit. An observed lack of such linearity in each of the predictors in this study resulted in each of the predictors being transformed to dichotomous scores based on changes observed in the model-development literature, according to the approach described elsewhere by Hosmer and Lemeshow (2000). Specifically, the criteria for the cut-points in decisional balance scores were based on the original hypothesis by Prochaska and colleagues that proposed that advances from preparation to action (inactive to active) are predicted by a $1.0 \mathrm{SD}$ increase in pros and a $.50 \mathrm{SD}$ decrease in cons (Prochaska et al., 1994). The criterion values for self-efficacy change (>.50 SD change in score), and change in behavioral ( $>.48$ SD change in score) and cognitive ( $>.32$ SD change in score) processes of change were taken from landmark studies applying the TTM to exercise (Marcus et al., 1998; Marcus, Rossi et al., 1992).

Univariate logistic regression analyses were then conducted for baseline to 3month, 3- to 6-month, and baseline to 6-month change scores in each independent variable as predictors for membership in the S-I or I-A groups. Any predictors with $p$ values less than or equal to .25 were then entered into the multivariate logistic regression model for each transition period (Hosmer \& Lemeshow, 2000). Backward elimination of the independent variables was performed until the model likelihood ratio reached a $p$ value less-than or equal to .05 . The strength of the predictors was examined using the Wald statistic, and overall model fit was assessed using the Hosmer-Lemeshow goodness-of-fit test. Odds ratios (OR) were examined for further indication of the strength of prediction of each independent variable. 


\section{Results - Demographics}

At baseline, 1600 survey invitations were sent via campus mail and email, though 250 were not deliverable. In all, 285 usable responses were collected at baseline out of a possible 1350 participants who were able to be reached (baseline response $21.1 \%$ ). A schematic representation of the baseline respondent and non-respondent categories can be found in Figure 4.1. No data were available to compare non-respondents to those who completed the baseline survey. A total of 159 participants $(55.8 \%)$ completed all three survey measurement points. The target sample were those participants who completed all three measurements, and were inactive at baseline $(N=60)$.

A brief description of the baseline characteristics of study participants used in this analysis $(N=60)$ can be found in Table 4.1. In addition to the frequency data, the average age was 41.59 years $(\mathrm{SD}=11.087)$, and $\mathrm{BMI}$ was $24.51(\mathrm{SD}=5.05)$. Participants were classified into the following "inactive" stages of change at baseline: PrecontemplationPC, $N=17$; Contemplation-C, $N=9$; Preparation-P, $N=34$. Comparisons of study dropouts (those who did not complete all three measurement points) who were in one of the inactive stages at baseline to those who completed the study revealed no significant differences in any baseline characteristics (all $p>.05$ ). Nominal and ordinal data for gender $(p=.619)$, education $(p=.643)$, marital status $(p=.540)$, job title $(p=.507)$, ethnicity $(p=.335)$, and baseline stage of change distribution $(p=.462)$ were compared using Chisquare analyses, and continuous data for age $(p=.546)$ and BMI $(p=.491)$ were compared using independent samples t-tests. 


\section{Results - Group Comparisons}

Because of the nature of the hypotheses of this study, the primary respondents of interest were the 60 participants who were classified in one of the inactive stages (PC, C, PR) at baseline and completed all three measurements (baseline, 3-month, 6-month). To corroborate the classification into the "inactive" stages, the average weekly total minutes of moderate and/or vigorous exercise reported by participants $(N=60)$ in the inactive stages at baseline $(61.73, S D=88.14)$ were compared to those in the active stages at baseline (273.52, $S D=174.08)$, and level of exercise was significantly different $(\mathrm{t}=-9.925$, $p<.0001$ ). Mean minutes of exercise by group (S-I, I-A) for those who were inactive at baseline can be found in Table 4.4 .

Means and standard deviations for each of the TTM-based constructs by adoption group (S-I vs I-A) at each measurement point (baseline, 3 months, 6 months) can be found in Table 4.2, and graphs of these data (with bars indicating standard error for each construct) can be found in Figures 4.2-4.6. Though not part of the primary analysis, Analyses of Variance (ANOVA) were conducted for each of the TTM constructs across all three measurements. $P$-values, effect size, and statistical power are listed for each analysis in Table 4.3. Additionally, correlations between the TTM constructs at each measurement point can be found in Table 4.5.

To further examine the time-course of change in behavior, a mixed-model $2 \times 3$ ANOVA was conducted comparing total weekly minutes of exercise (vigorous and/or moderate minutes) for those in the S-I group to those in the I-A group. A significant interaction effect $\left(\mathrm{F}=4.167, p=.020\right.$, partial $\eta^{2}=.118$, Power=.673) was found. 
Post-hoc t-tests revealed no significant differences between baseline and 3-month minutes $(\mathrm{t}=-1.279, \mathrm{p}=.219)$ or between 3 - and 6-month minutes $(\mathrm{t}=-1.525, p=.147)$ in the I-A group, but minutes of exercise at 6-months were significantly greater than those reported at 3-months $(\mathrm{t}=-2.955, p=.009)$. No significant differences were found between baseline and 3-month minutes $(\mathrm{t}=.389, p=.699)$, between 3 - and 6-month minutes $(\mathrm{t}=.066$, $\mathrm{p}=.948)$, or baseline and 6-month minutes $(\mathrm{t}=.312, \mathrm{p}=.757)$ for the S-I group.

Post-hoc independent samples t-tests were conducted between the S-I and I-A groups at each measurement point, and no significant differences in weekly minutes were found at baseline $(\mathrm{t}=-.405, p=.687)$ or at three-months $(\mathrm{t}=-2.204, p=.32)$. At 6 -months, minutes of exercise per week were significantly greater for the I-A group $(t=-3.310$, $p=.002$ ). Table 4.4 and Figure 4.7 show mean weekly minutes of exercise by group and time of measurement.

\section{Results - Logistic Regression}

As a first step in model-testing, univariate logistic regression analyses were conducted using the dichotomous transformation of change scores (either above or below the criterion level of change) for each of the TTM constructs (B-POC, C-POC, SE, DBPro, DB-Con) as the predictor and adoption group (S-I, I-A) as the outcome. Criteria for entry into the multivariate model were consistent with those proposed by Hosmer and Lemeshow (2000), specifically, having a Wald statistic $p$-value less than or equal to .25. For BL to 3-month changes in TTM constructs, B-POC was the only predictor identified to enter into the multivariate model of prediction for adoption $(p=.028, \mathrm{OR}=12.923$, Model $\chi^{2}=6.371$, Model $p=.012$ ). For the model based on 3- to 6-month changes in TTM constructs, both B-POC $\left(p=.027, \mathrm{OR}=4.317\right.$, Model $\chi^{2}=4.930$, Model $\left.p=.026\right)$ and SE 
$\left(p=.045, \mathrm{OR}=3.297\right.$, Model $\chi^{2}=4.143$, Model $\left.p=.042\right)$ were identified as qualifying for entry into the multivariate model. For the model based on BL to 6-month changes in TTM constructs, B-POC ( $p=.026, \mathrm{OR}=4.317$, Model $\chi^{2}=4.930$, Model $\left.p=.027\right)$, SE $\left(p=.075, \mathrm{OR}=2.933\right.$, Model $\chi^{2}=3.154$, Model $\left.p=.076\right), \mathrm{C}-\mathrm{POC}(p=.046, \mathrm{OR}=3.600$, Model $\chi^{2}=3.951$, Model $\left.p=.047\right)$, and DB-Con $\left(p=.25, \mathrm{OR}=.388\right.$, Model $\chi^{2}=1.501$, Model $p=.220$ ) were identified for entry into the multivariate model. All other TTM constructs had $p$-values above .25, and were not entered into the multivariate models for their respective time-period.

Multivariate regression analyses were conducted for all of the above-mentioned time periods. For the BL to 3-month changes, only B-POC entered into the prediction, and the values remained the same as in the individual regression equation $(\mathrm{OR}=12.923$, Model $\chi^{2}=6.371$, Model $p=.012$ ). For 3- to 6-month changes in TTM processes, both B$\operatorname{POC}(p=.023, \mathrm{OR}=4.986)$ and $\mathrm{SE}(p=.038, \mathrm{OR}=3.777)$ and were retained in the model (Model $\chi^{2}=9.499$, Model $\left.p=.009\right)$. For BL to 6-month changes, C-POC ( $p=.057$, $\mathrm{OR}=3.730), \mathrm{SE}(p=.162, \mathrm{OR}=2.443)$, and DB-Con $(p=.214, \mathrm{OR}=.332)$ were retained in the model (Model $\chi^{2}=8.062$, Model $p=.045$ ). Though the individual variables retained in the BL to 6-month model were not significant, each contributed to the significant overall multivariate model which was determined using the model likelihood statistic. Odds ratios were computed for each of the retained predictors in the three models. Figure 4.8 is a graphic representation of the odds ratios for each of TTM constructs in the multivariate model prediction equations. 


\section{Discussion}

The main purpose of this study was to examine the ability of all TTM constructs to predict the adoption of active stage over a 6-month measurement period. Specifically, it was hypothesized that predictions based on baseline to 6-month changes in the TTM constructs would be strongest, while baseline to 3-month changes and 3- to 6-month changes would be weaker predictors of adoption of an active stage. It is important to note at the outset that the findings of this study should be considered preliminary due to the small sample size $(N=60)$, which resulted in low statistical power, the collapse of stages into inactive and active categories, and the dichotomous transformation of the predictors.

We found that over a 6-month follow-up, self-efficacy, decisional balance-cons, and cognitive processes of change were all significant predictors of the active adoption by 6 months. Of these predictors, odds ratios and $p$-values indicated that the cognitive processes of change were the strongest contributor to the model, followed by selfefficacy and decisional balance-cons. Our finding that the cognitive processes of change were the strongest predictor in the baseline to 6-month model was inconsistent with traditional TTM assumptions. This finding is also in contrast with several longitudinal studies, (Higgins \& Oldenburg, 2003; Maddison \& Prapavessis, 2006; O'Hea et al., 2004) that reported self-efficacy to be the most consistent predictor of stage transition. One study by Rhodes \& Plotnikoff (2006) reported that all of the TTM constructs significantly discriminated between various intention profiles, though self-efficacy did not discriminate those adopting the behavior from the other profiles over the 6-month followup, and only decisional balance-cons were able to discriminate those successfully adopting exercise from those who were unsuccessful. 
More information regarding prediction of stage transition was revealed when the 3-month changes in TTM processes were examined, both from baseline to 3 months and from 3 to 6 months. We found that baseline to 3-month changes in behavioral processes of change were the only significant predictor for adoption of an active stage from baseline to 6-months. Changes in behavioral processes of change and self-efficacy were both retained in the 3- to 6-month multivariate model. An examination of the odds ratios (OR) revealed that those individuals who increased their use of the behavioral processes of change by the criteria amount (.48 points on the processes of change instrument) in the first 3 months were 12.92 times more likely to adopt an active stage by 6-months than participants who did not. Those who met the criterion level of change in behavioral processes from 3- to 6-months, were 4.99 times more likely to adopt an active stage by 6months. The odds of adopting an active stage by 6 months were 3.78 times greater for those participants who increased their self-efficacy by the criterion amount (.40 points on the self-efficacy instrument) during the final 3 months of the study.

Further examination of the descriptive data in Figure 4.9 revealed deeper information about the time course of change in the TTM constructs. Specifically, much of the 6-month change in self-efficacy for those in the I-A group appears to have occurred in the final 3 months of the study. It is possible that the important baseline to 3-month changes in behavioral processes of change may have led to higher levels of self-efficacy during the later measurement periods, as other research into process use has identified early changes in the behavioral process of change as being important in stage transition (Lewis et al., 2006; Plotnikoff et al., 2001). These findings are in contrast to the theorized relationship between processes of change and stage transition, and may be representative 
of another construct underlying the timeline of adoption. Perhaps those who improve in the use of certain TTM constructs in less time than 6 months are early adopters, and have a different set of behavioral characteristics than those who take longer (6-months).

Interestingly, both the baseline to 3-month and 3- to 6-month regression equations contained the behavioral processes of change as a significant predictor of adoption, but this construct disappeared when the full baseline to 6-month changes were considered. One possible explanation is that the critical changes in behavioral process use are over before the full 6-month timeline is completed. Again, this is in contrast with the hypothesized use of behavioral processes late in stage movement (Prochaska \& Velicer, 1997b). On the other hand, it is possible that a full 6-month of change in the cognitive processes of change and decisional balance-cons are needed to influence adoption.

Examining the correlations between TTM constructs at all measurement points reveals that the TTM constructs are highly inter-correlated, but that some of the smaller correlations are between the cognitive processes of change and self-efficacy. It is possible that the higher amounts of unique variance explained by the cognitive processes of change may have contributed to the predictive ability in the baseline to 6-month model. Consequently, the higher levels of correlation between self-efficacy and the behavioral processes of change may have served to cancel out some of the effects of behavioral processes of change over the full 6-months, which may have contributed to the elimination of this construct from the 6-month model.

A meta-analysis of the TTM in exercise by Marshall and Biddle (2001) and a critical review of stage models by Weinstein and colleagues (1998) both called for further examination of the TTM using more advanced designs to overcome the limitations of 
cross-sectional examinations. Our study attempted to answer some of these criticisms by examining naturalistic stage progression over a 6-month follow-up, with intermittent measures to provide a more detailed picture of the time-course of change in TTM constructs and their contribution to exercise adoption.

Overall, these findings cast some doubt upon the importance of the 6-month marker in the TTM for exercise, and expose a temporal weakness in the TTM. The TTM proposes that the critical time factors in the behavior change process are either immediate (around 1 month for the preparation stage) or 6 months or longer (precontemplation, contemplation, action, maintenance stages), and little attention is paid to the critical variables that would predict changes over a shorter period of time. As evidence, our study found different predictors of change when the time-course that was considered was shortened to 3 months. This indicates that examinations focusing simply on the behavior change process in 6-month blocks miss important information about critical times for change, as well as which processes provide the most explained variance in stage transition. If our study had not examined the 3-month increments, behavioral process of change use would not have been considered to be important because it was not significant in the multivariate baseline to 6-month regression model. Instead, we found that early use of the behavioral processes is a strong predictor of movement to an active stage over 6-months.

Given the estimates that over $50 \%$ of participants drop out of exercise programs within the first 6-months, it seems useful to incorporate within the model an explanation for this observed behavior and construct use over shorter and perhaps more critical time periods. In terms of self-reported minutes of exercise, the increase from BL to 3-months 
in the I-A group only approached significance ( $p=.06)$, but the effect size (Cohen's $d=.47$ ) implies that important changes in behavior occur within the benchmark 6-months stage period. This casts some doubt upon the homogeneity of reaching the action stage within a 6-month time period. It is also interesting to note that those in the S-I group were classified into one of the inactive stages for the purposes of this study, but averaged more than 50 minutes per week of exercise (Table 4.3) throughout the study. This may have resulted in miss-classification of some individuals into inactive stages.

This study had several strengths that made it a unique contribution to the TTM literature. First, the longitudinal design of the study allowed the analysis of change scores over time, which provided more information about the time-course of change for the TTM constructs and their ability to predict those who moved to an active stage of change by 6 months. Two separate reviews of the TTM have suggested that the utility of cross-sectional examinations of the model have likely run their course, and that among others, longitudinal designs should be utilized to more fully test the predictive nature of the model (Marshall \& Biddle, 2001; Weinstein et al., 1998). Other longitudinal studies were reviewed earlier in this paper, however, none of these examined naturalistic changes in TTM constructs over a shorter period than 6-months. This study extended the findings of others that identified the importance of the behavioral processes of change in early stage transition (Lewis et al., 2006; Plotnikoff et al., 2001), and supported the importance of self-efficacy in adoption of an active stage.

Second, changes in physical activity mirrored the change in stage type over the 6month follow-up. While groups reported similar amounts of exercise at baseline, at 6months those in the I-A group averaged 162.65 total minutes exercise per week, which 
was significantly different from that reported by those in the S-I group (52.79 minutes) $p<.05$. The time spent exercising reported at 6 months by the I-A group was significantly higher than baseline total weekly minutes of exercise. Levels of exercise reported provide corroboration that the adoption of an active stage was also manifested as a change in behavior, with the I-A group averaging significantly higher minutes than the S-I group, and falling within the recommended weekly amount and intensity of exercise according to the ACSM (1998).

A third strength of the study was the lack of significant differences in baseline demographic characteristics between those who remained in the study throughout the 6month follow-up and those who dropped out during the study. This allowed for greater generalization to a population with similar demographic characteristics as this sample. Additionally, the retention rate for the study was rate was $55.8 \%$, which was higher than expected based on a study of similar design (Plotnikoff et al., 2001).

There were several important limitations of the study that may have affected the findings. One of the primary limitations was the small sample size and low response rate. Because many of the respondents to the initial mailing were already active, they were not included in the analysis. In the end, 60 participants met the criteria for inclusion in the study, and 17 of these were classified into the I-A group. This was well below the 168 participants in the I-A group that we had initially hoped to have. This small sample size was reflected in the low statistical power shown in Table 4.3. Only one of the analyses had a statistical power over .8, the standard for ANOVA (Keppel, 1991). As a consequence, some of the larger effect sizes observed in these analyses did not reach statistical significance. A primary example of this was self-efficacy, which had a within- 
groups effect size of .70, which was equal to the within-groups effect size seen in the behavioral processes of change. Because of differing power between the two analyses $(\mathrm{SE}=.42$, $\mathrm{B}-\mathrm{POC}=.69)$, the within-groups factor for behavioral processes of change was statistically significant $(p=.02)$ where the within-groups factor for self-efficacy was not $(\mathrm{p}=.13)$. A larger sample size would have overcome some of the variability in these constructs and created more potential for finding significance where appropriate. This small sample size likely limited the potential for finding significance among predictors in the logistic regression analyses as well. Therefore, it is important that these results are taken as preliminary examinations in light of the limitations that our small sample size created.

Cut-points in the logistic regression analysis were useful in interpretation of the results, but these values were based on review of key studies, and there is no consensus on amount of change in TTM constructs that would predict a change in stage, aside from the "Strong Principal of Progress" regarding 1.0 SD increases in pros across stages proposed by Prochaska $(2006 ; 1994)$. Part of the cause for this is the lack of valid modeltesting of the TTM in exercise, and also a lack of reporting of raw scores for the TTM constructs. As outlined in the methods section of this paper, cut-points were established based on the developmental literature for the TTM in exercise in order to be consistent with the model-testing nature of this study. Despite the attempt to utilize appropriate criteria for developing these cut-points, a change in criteria may have altered the findings contained within this study. Though our findings differed from some of the traditional hypothesized relationships among TTM constructs, enough consistency with previous 
literature (especially the importance of self-efficacy) was noted to also provide a sense of justification in the decision-making process.

Another potential limitation was the collapsing of the stages of change.

Combining the precontemplation, contemplation, and preparation stages into the "inactive" category and the action and maintenance stages into the "active" category was necessary to preserve power in the study, given the relatively low number of respondents per construct. Because of this limitation, it is important that future research examines the factors that are most predictive of transition into and out of specific stages of change, as the TTM proposes that each of the stages have distinct barriers and processes. To fully test the model, however, it would be necessary to have sufficient numbers of respondents in each stage of change, which has proven to be difficult. Every reviewed study that examined stage transition used some sort of collapsed stage format because of issues with finding sufficient power to test the stages individually, and ours was no exception.

Despite the good retention rate of our study, the overall sample size for the I-A group was lower than was expected. This was partially due to the large proportion of baseline participants (61.05\%) classified in the active stages. Both active and inactive participants were targeted at the outset of the study in order to maximize initial response and to provide a sample for future inquiry into other TTM dynamics. Having such a highly active baseline sample, however, resulted in the low sample size in the I-A group. This reflects the ongoing difficulty in exercise behavior research of recruiting sufficient numbers of inactive subjects.

In addition to activity levels, other demographic characteristics of this sample may have limited our findings. Table 4.1 provides demographic details, but of concern to 
the generalizability of these findings to the greater population were the racial, educational, marital, and professional characteristics. This was primarily a highly educated, professional, white sample, and these factors have been consistently found to be related to higher-than-average levels of physical activity (U.S. Department of Health and Human Services, 1996). The TTM constructs that predicted behavior change in this sample may not generalize to other demographics, particularly those identified as potential intervention targets due to lower activity rates.

Finally, this study was conducted between September and March and as such the autumn and winter season may have had an effect on lowering participants' activity rates. Previous research has shown that season may have an effect on various levels of leisuretime physical activity (Matthews et al., 2001), with higher activity rates in the warmer months. Though the design of this study precluded using season as a predictor, the singlelocation used in this study meant that all participants were subject to the same seasonal effects. Additionally, any significant findings in relation to season would be an indication that the model does not sufficiently account for season as an environmental predictor of stage transition.

Future studies can overcome some of the limitations of this study in several ways. First, over-sampling of the inactive stages of change could maximize the potential for a larger sample size, and for examining the transition out of each inactive stage of change between baseline and follow-up, rather than collapsed stages. Because of the prevalence of inactivity in underrepresented demographic groups, over-sampling of inactive individuals may also result in a more representative sample, unlike that which was obtained in this study. Additionally, more frequent assessment may be necessary to 
capture the dynamics of a repetitive behavior like physical activity. Perhaps obtaining one measurement per month would allow for better elucidation of key variables, more data about the time-course of change, and more consistent prediction by the constructs contained within the TTM.

In conclusion, though the findings of this study cast some preliminary doubt on the seemingly rigid 6-month behavioral definitions contained within the TTM, more work needs to be done to examine the stages individually to determine which processes are important in the transition out-of and into each of the stages. Furthermore, rigorous longitudinal studies of stage transition will inform future interventions about the most efficacious constructs on which to focus for each stage of change, and whether the duration of stages should be modified. It is also important to incorporate stage-matched and mismatched intervention studies with balanced numbers of inactive and active subjects to extend the findings of longitudinal studies and provide a deeper understanding of the dynamics of behavior change in light of relationships between TTM constructs (Weinstein et al., 1998). These designs test the effectiveness of theoretically-based interventions against an intervention which is purposefully designed to contradict the hypothesized relationships between theoretical variables and the dependent variable. 


\begin{tabular}{lcc}
\hline Demographic Variable & $\mathrm{N}$ & $\%$ \\
\hline Gender & 18 & 30.0 \\
Male & 41 & 68.3 \\
Female & 1 & 1.7 \\
N/A & & \\
Ethnicity & 51 & 85.0 \\
White & 6 & 3.0 \\
Black / African American & 3 & 5.0 \\
Asian & & \\
Marital Status & 32 & 53.3 \\
Married & 13 & 21.7 \\
Never Married & 7 & 11.7 \\
Divorced & 2 & 3.3 \\
Separated & 5 & 8.3 \\
Member of an Unmarried Couple & 1 & 1.7 \\
Prefer Not to Answer & & \\
Highest Level of Education & 5 & 8.3 \\
Graduated from High School & 5 & 8.3 \\
Some Education After High School/GED & 2 & 3.3 \\
Associate's Degree & 26 & 43.3 \\
Bachelor's Degree & 10 & 16.7 \\
Master's Degree & 2 & 3.3 \\
Professional Degree & 5 & 8.3 \\
Doctoral Degree & 5 & 8.3 \\
Post-doctoral Training & & \\
\hline
\end{tabular}

Table 4.1 Demographic Characteristics of Target Sample $(N=60)$ 


\begin{tabular}{|c|c|c|c|c|c|c|c|}
\hline \multirow[b]{2}{*}{ Group } & \multirow[b]{2}{*}{ Construct } & \multicolumn{2}{|c|}{ Baseline } & \multicolumn{2}{|c|}{ 3-Month } & \multicolumn{2}{|c|}{ 6-Month } \\
\hline & & Mean & $\mathrm{SD}$ & Mean & $\mathrm{SD}$ & Mean & $\mathrm{SD}$ \\
\hline \multicolumn{8}{|l|}{ S-I } \\
\hline & $\mathrm{SE}^{\mathrm{a}}$ & 2.261 & .802 & 2.209 & .750 & 2.214 & .639 \\
\hline & C-POC & 2.705 & .638 & 2.683 & .606 & 2.767 & .662 \\
\hline & $\mathrm{B}-\mathrm{POC} \mathrm{C}^{\mathrm{b}, \mathrm{c}}$ & 2.121 & .472 & 2.084 & .578 & 2.108 & .535 \\
\hline & DB-Pros & 3.616 & .955 & 3.480 & .985 & 3.686 & .857 \\
\hline & DB-Cons ${ }^{e}$ & 2.609 & .637 & 2.690 & .606 & 2.767 & .564 \\
\hline \multicolumn{8}{|l|}{ I-A } \\
\hline & $\mathrm{SE}^{\mathrm{a}}$ & 2.541 & .758 & 2.647 & .808 & 2.952 & .896 \\
\hline & C-POC & 2.953 & .722 & 3.003 & .730 & 3.085 & .751 \\
\hline & B-POC ${ }^{b, c, d}$ & 2.391 & .518 & 2.585 & .695 & 2.782 & .726 \\
\hline & DB-Pros & 3.718 & .913 & 3.759 & .978 & 3.712 & .715 \\
\hline & DB-Cons ${ }^{e}$ & 2.363 & .602 & 2.294 & .730 & 2.314 & .770 \\
\hline
\end{tabular}

Table 4.2 TTM Construct Means and Standard Deviations for S-I $(N=43)$ and I-A $(N=17)$ Participants

Note: $\mathrm{S}-\mathrm{I}=$ Stable-Inactive, $\mathrm{I}-\mathrm{A}=$ Inactive-Active ${ }^{\mathrm{a}} \mathrm{I}-\mathrm{A}>\mathrm{S}-\mathrm{I}$ at 6 -month $(\mathrm{p}<.05) ;{ }^{\mathrm{b}} \mathrm{I}-\mathrm{A}>$ S-I at 3-month and 6-month $(\mathrm{p}<.05) ;{ }^{\mathrm{c}} 6$-month $>3$-month in I-A group $(\mathrm{p}<.05) ;{ }^{\mathrm{d}} 6$ month $>$ baseline for I-A group $(\mathrm{p}<.05) ;{ }^{\mathrm{e}}$ main effect S-I vs I-A $(\mathrm{p}<.05)$ 


\begin{tabular}{|c|c|c|c|c|}
\hline Construct & & P-value & Effect Size $^{\mathrm{a}}$ & Power \\
\hline \multicolumn{5}{|l|}{$\mathrm{SE}$} \\
\hline & Within & .125 & .07 & .42 \\
\hline & Between & .014 & .10 & .71 \\
\hline & Interaction & .050 & .10 & .58 \\
\hline \multicolumn{5}{|l|}{ Cog-POC } \\
\hline & Within & .197 & .03 & .34 \\
\hline & Between & .103 & .05 & .37 \\
\hline & Interaction & .763 & .01 & .09 \\
\hline \multicolumn{5}{|l|}{ Beh-POC } \\
\hline & Within & .022 & .07 & .69 \\
\hline & Between & .001 & .16 & .91 \\
\hline & Interaction & .013 & .08 & .75 \\
\hline \multicolumn{5}{|l|}{ DB-Pro } \\
\hline & Within & .671 & .01 & .11 \\
\hline & Between & .577 & .01 & .09 \\
\hline & Interaction & .353 & .02 & .23 \\
\hline DB-Con & Within & .689 & .01 & .11 \\
\hline & Between & .027 & .08 & .61 \\
\hline & Interaction & .333 & .02 & .24 \\
\hline
\end{tabular}

Table 4.3 P-values, Effect Size, and Power for 2x3 (Group x Time) Mixed Model ANOVA on Each TTM Construct

Note: ${ }^{a}$ Effect size statistic is given as partial $\eta^{2}$; $\mathrm{SE}=$ Self-Efficacy; Cog-POC=Cognitive Processes of Change; Beh-POC $=$ Behavioral Processes of Change; DB-Pro=Decisional Balance-Pros; DB-Con=Decisional Balance-Cons 


\begin{tabular}{lcccccc}
\hline & \multicolumn{2}{c}{ Baseline } & \multicolumn{2}{c}{ 3-Month } & \multicolumn{2}{c}{ 6-Month $^{\mathrm{a}}$} \\
\cline { 2 - 7 } Group & Mean & SD & Mean & SD & Mean & SD \\
\hline S-I & 58.81 & 93.02 & 54.07 & 90.80 & 52.79 & 116.237 \\
I-A $^{\text {b }}$ & 69.12 & 76.51 & 117.35 & 121.59 & 162.65 & 114.784 \\
\hline
\end{tabular}

Table 4.4 Mean and Standard Deviation (SD) of Total Weekly Minutes of Exercise for SI $(N=43)$ and I-A $(N=17)$ Participants by Measurement Time

Note: $\mathrm{S}-\mathrm{I}=$ Stable-Inactive, $\mathrm{I}-\mathrm{A}=$ Inactive-Active; ${ }^{\mathrm{a}} \mathrm{I}-\mathrm{A}>\mathrm{S}-\mathrm{I}$ at $6-$ month $(\mathrm{p}<.05) ;{ }^{\mathrm{b}} 3$ month $>6$-month for I-A $(\mathrm{p}<.05)$ 


\begin{tabular}{|c|c|c|c|c|c|c|c|c|c|c|c|c|c|c|}
\hline & $\begin{array}{c}\text { DB PRO } \\
\text { BL }\end{array}$ & $\begin{array}{c}\text { DB } \\
\text { CON } \\
\text { BL } \\
\end{array}$ & $\begin{array}{c}\text { C-POC } \\
\text { BL }\end{array}$ & $\begin{array}{c}\text { B-POC } \\
\text { BL }\end{array}$ & $\begin{array}{c}\text { SE } \\
\text { 3-MO }\end{array}$ & $\begin{array}{c}\text { DB PRO } \\
\text { 3-MO }\end{array}$ & $\begin{array}{c}\text { DB } \\
\text { CON } \\
\text { 3-MO } \\
\end{array}$ & $\begin{array}{c}\text { C-POC } \\
\text { 3-MO } \\
\end{array}$ & $\begin{array}{c}\text { B-POC } \\
\text { 3-MO } \\
\end{array}$ & $\begin{array}{c}\text { SE } \\
\text { 6-MO }\end{array}$ & $\begin{array}{c}\text { DB PRO } \\
6 \text { MO } \\
\end{array}$ & $\begin{array}{c}\text { DB } \\
\text { CON } \\
6 \text { MO } \\
\end{array}$ & $\begin{array}{l}\text { C-POC } \\
\text { 6-MO } \\
\end{array}$ & $\begin{array}{l}\text { B-POC } \\
\text { 6-MO } \\
\end{array}$ \\
\hline SE BL & .201 & $-.508(* *)$ & $.304(*)$ & $.554(* *)$ & $.722(* *)$ & .158 & $-.363(* *)$ & .205 & $.400(* *)$ & $.647(* *)$ & .096 & $-.512(* *)$ & .159 & $.404(* *)$ \\
\hline DB PRO BL & & -.064 & $.768(* *)$ & $.458(* *)$ & .202 & $.800(* *)$ & -.054 & $.672(* *)$ & $.373(* *)$ & .202 & $.742(* *)$ & .003 & $.566(* *)$ & .229 \\
\hline DB CON BL & & & -.162 & $-.268\left(^{*}\right)$ & $-.427(* *)$ & -.061 & $.683(* *)$ & -.116 & $-.347(* *)$ & $-.593(* *)$ & -.073 & $.732(* *)$ & -.132 & $-.409(* *)$ \\
\hline C-POC BL & & & & $.633(* *)$ & $.348(* *)$ & $.667(* *)$ & -.046 & $.835(* *)$ & $.591(* *)$ & $.380(* *)$ & $.598(* *)$ & -.070 & $.793(* *)$ & $.419(* *)$ \\
\hline B-POC BL & & & & & $.470(* *)$ & $.463(* *)$ & -.117 & $.527(* *)$ & $.701(* *)$ & $.459(* *)$ & $.269\left(^{*}\right)$ & -.219 & $.447(* *)$ & $.580(* *)$ \\
\hline SE 3-MO & & & & & & .251 & $-.455(* *)$ & $.327(*)$ & $.577(* *)$ & $.646(* *)$ & .106 & $-.390(* *)$ & .225 & $.471(* *)$ \\
\hline DB PRO 3-MO & & & & & & & -.028 & $.755(* *)$ & $.489(* *)$ & .168 & $.764(* *)$ & .003 & $.682(* *)$ & $.417(* *)$ \\
\hline DB CON 3-MO & & & & & & & & -.048 & -.247 & $-.494(* *)$ & -.098 & $.685(* *)$ & -.039 & $-.273(*)$ \\
\hline C-POC 3-MO & & & & & & & & & $.689(* *)$ & $.329\left(^{*}\right)$ & $.579(* *)$ & -.062 & $.854(* *)$ & $.567(* *)$ \\
\hline B-POC 3-MO & & & & & & & & & & $.538(* *)$ & $.275\left(^{*}\right)$ & $-.273\left(^{*}\right)$ & $.623(* *)$ & $.824(* *)$ \\
\hline SE 6-MO & & & & & & & & & & & .188 & $-.602(* *)$ & $.267\left(^{*}\right)$ & $.635(* *)$ \\
\hline DB PRO 6-MO & & & & & & & & & & & & .080 & $.683(* *)$ & $.284(*)$ \\
\hline DB CON 6-MO & & & & & & & & & & & & & -.019 & $-.411(* *)$ \\
\hline C-POC 6-MO & & & & & & & & & & & & & & $.584(* *)$ \\
\hline
\end{tabular}

Table 4.5 Correlation Matrix of All TTM Constructs at Each Measurement Point $(N=60)$

Note: ** Correlation is significant at the .01 level (2-tailed); * Correlation is significant at the .05 level (2-tailed); SE=SelfEfficacy; DB-PRO=Decisional Balance-Pros; DB-CON=Decisional Balance-Cons; C-POC=Cognitive Processes of Change; B$\mathrm{POC}=$ Behavioral Processes of Change; $\mathrm{BL}=$ Baseline; $3-\mathrm{MO}=$ Three Months; $6-\mathrm{MO}=$ Six Months 


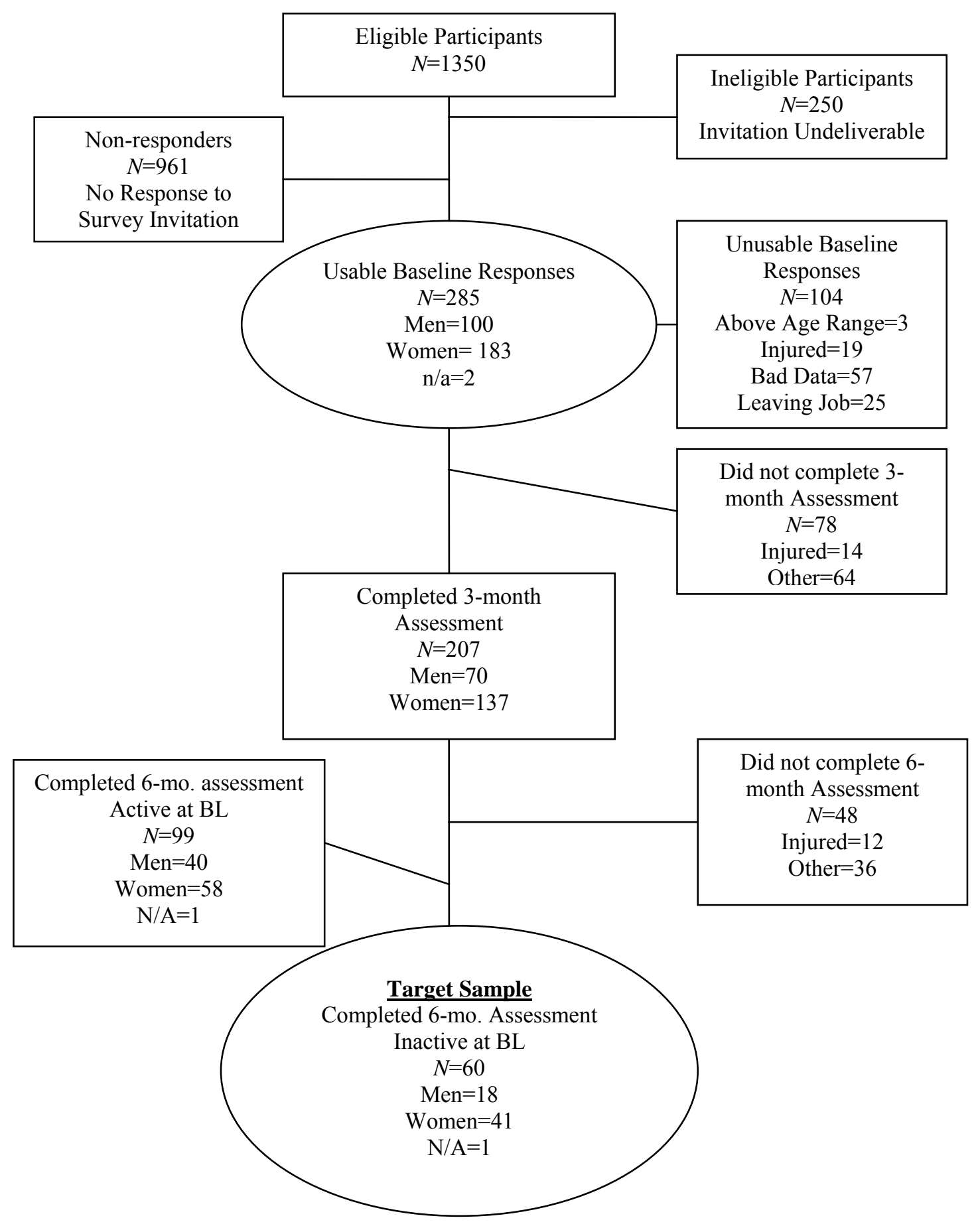

Figure 4.1 Participant Flow Chart 


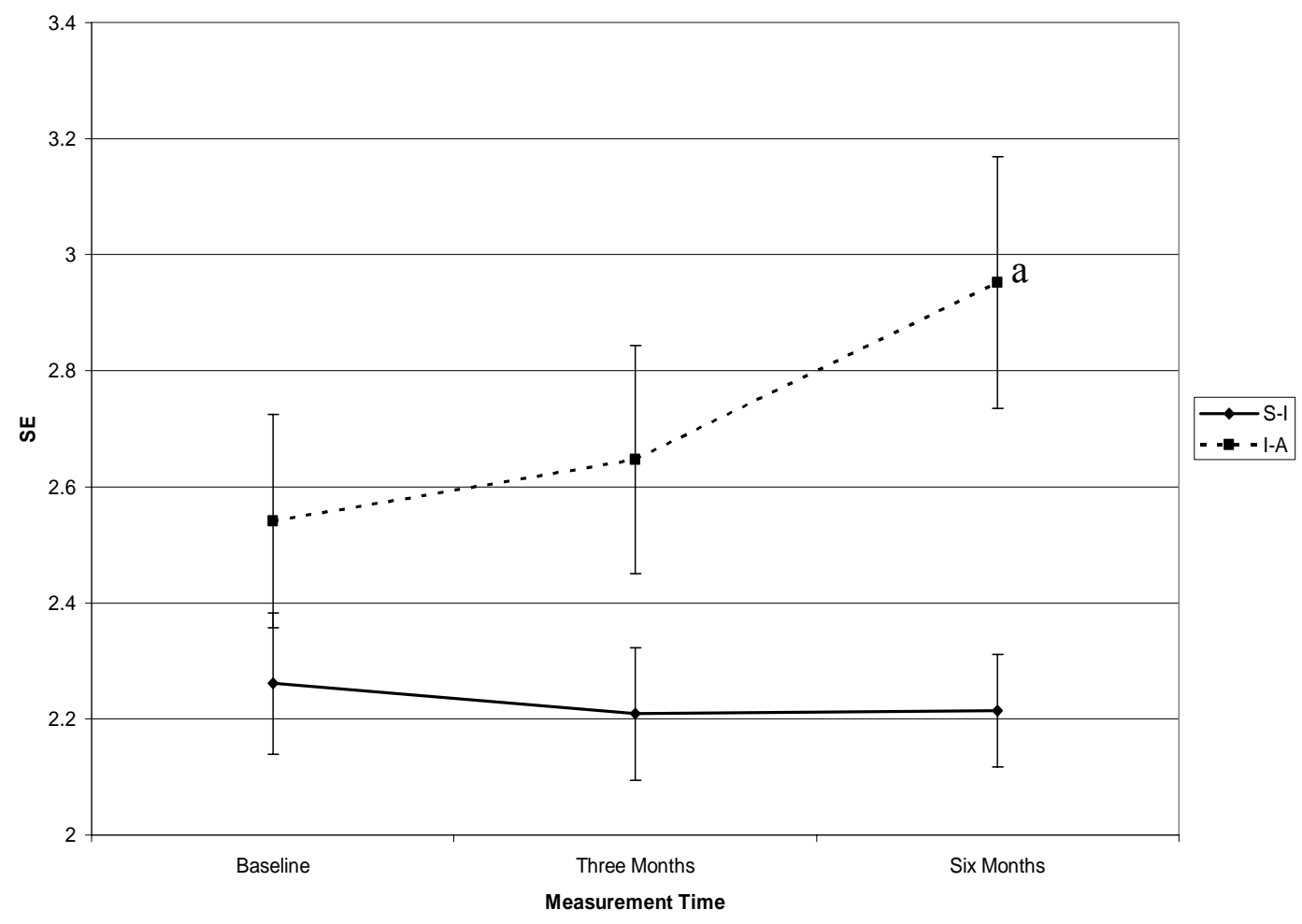

Figure 4.2 Mean Self-Efficacy Scores for S-I $(N=43)$ and I-A $(N=17)$ Groups by Measurement Time

Note: Error bars are \pm 1 S.E.; S-I $=$ Stable-Inactive, I-A $=$ Inactive-Active; ${ }^{\text {a }}$ I-A $>$ S-I at Six Months $(\mathrm{p}<.05)$ 


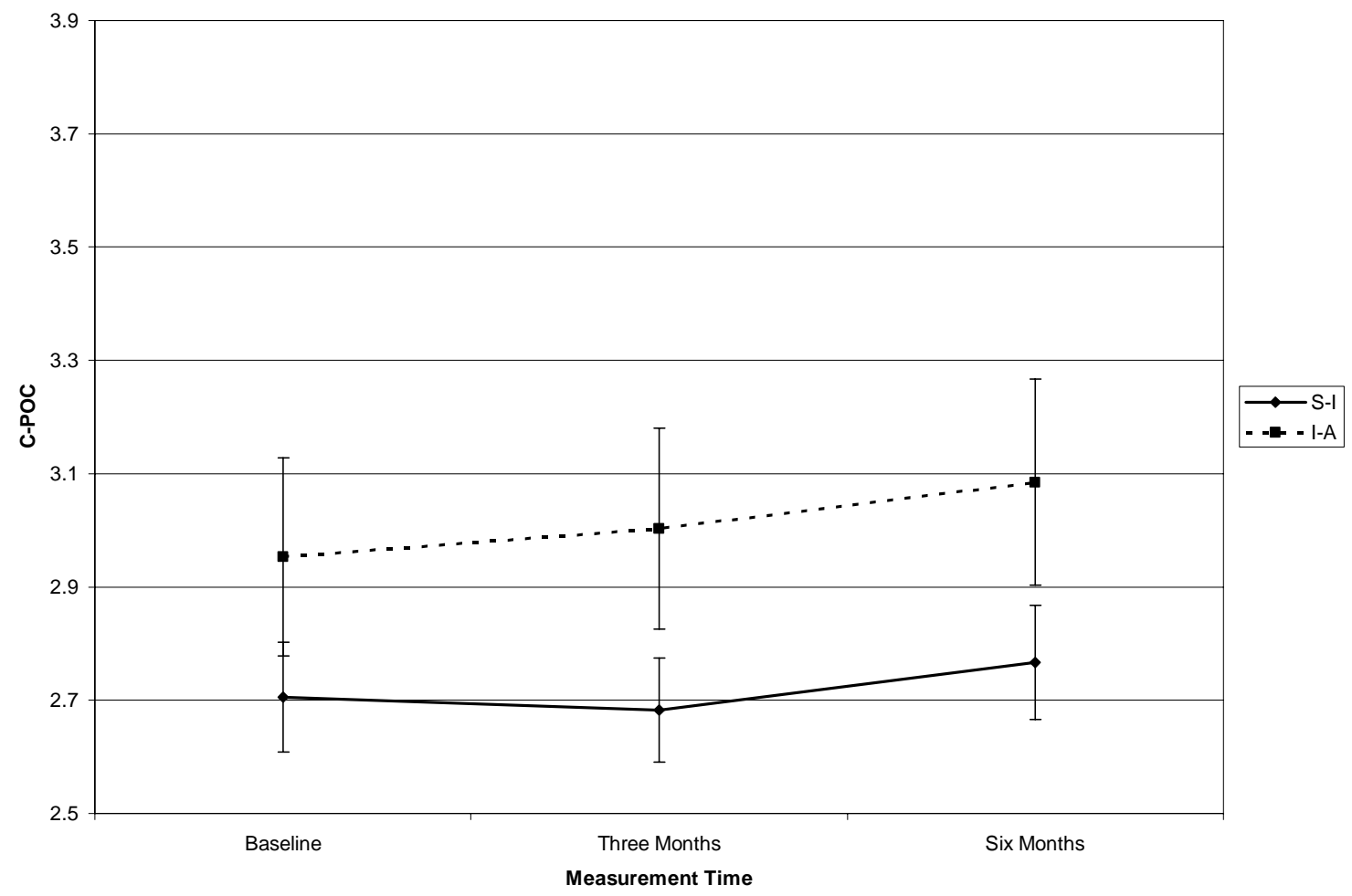

Figure 4.3 Mean Cognitive Processes of Change Scores for S-I $(N=43)$ and I-A $(N=17)$ Groups by Measurement Time

Note: Error bars are \pm 1 S.E.; S-I = Stable-Inactive, $\mathrm{I}-\mathrm{A}=$ Inactive-Active 


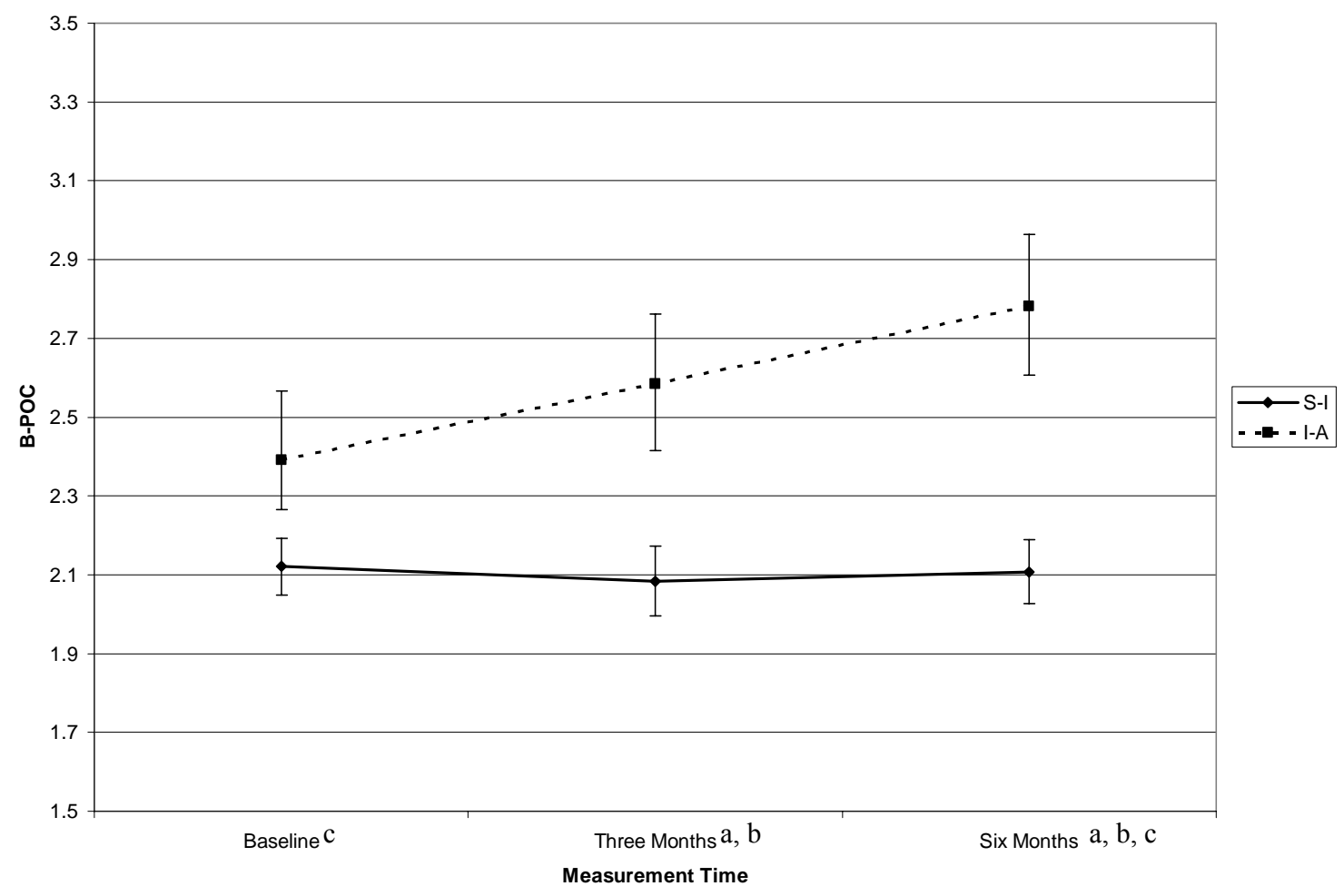

Figure 4.4 Mean Behavioral Processes of Change Scores for S-I $(N=43)$ and I-A $(N=17)$ Groups by Measurement Time

Note: Error bars are \pm 1 S.E.; S-I $=$ Stable-Inactive, I-A = Inactive-Active; ${ }^{a}$ I-A $>$ S-I at Three Months and at Six Months $(\mathrm{p}<.05) ;{ }^{b}$ Three Months $>$ Six Months in I-A group $(\mathrm{p}<.05) ;{ }^{\mathrm{c}}$ Six Months $>$ Baseline in I-A group $(\mathrm{p}<.05)$ 


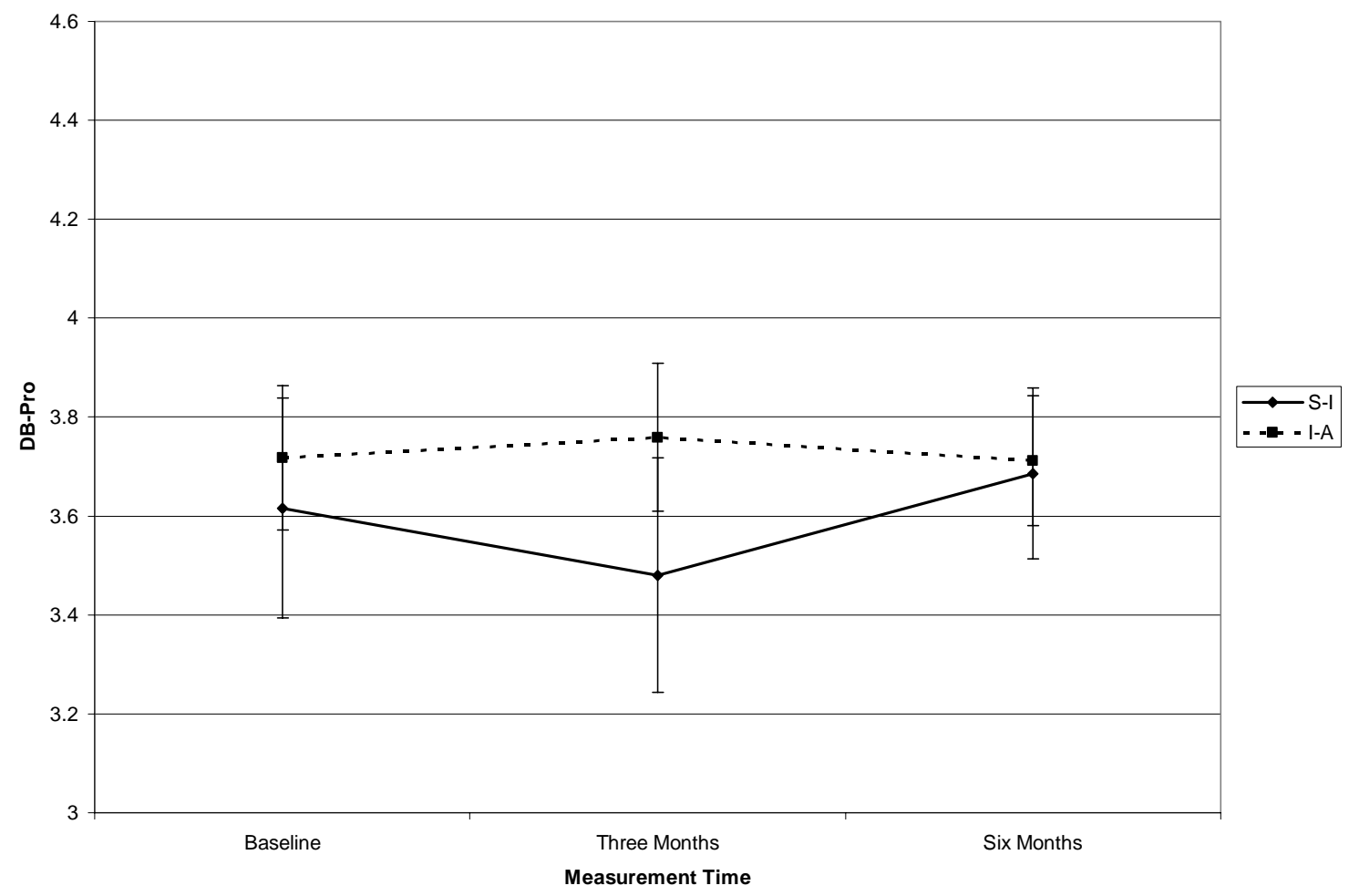

Figure 4.5 Mean Decisional Balance - Pro Scores for S-I $(N=43)$ and I-A $(N=17)$ Groups by Measurement Time

Note: Error bars are \pm 1 S.E.; S-I = Stable-Inactive, $\mathrm{I}-\mathrm{A}=$ Inactive-Active 


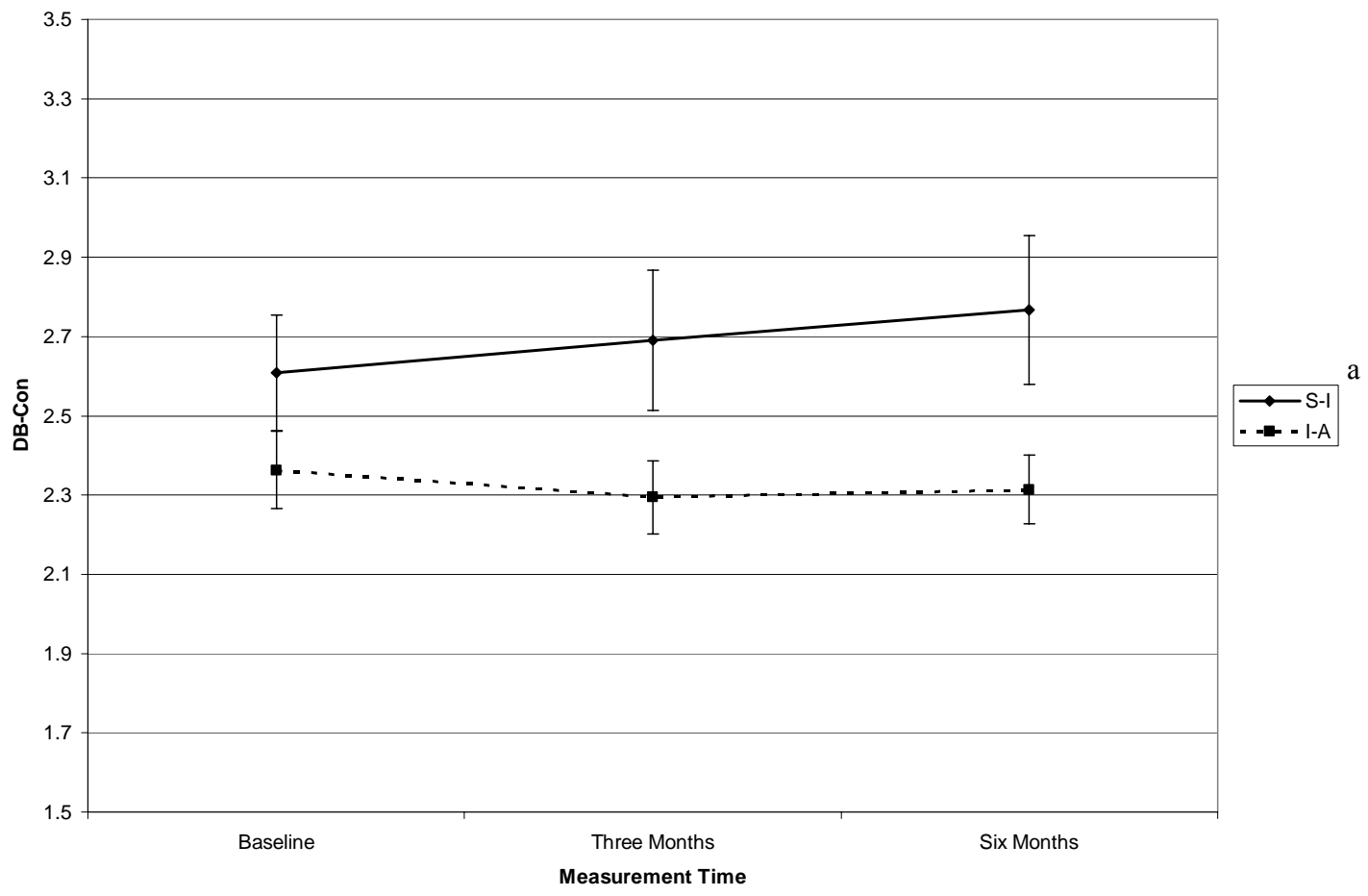

Figure 4.6 Mean Decisional Balance - Con Scores for S-I $(N=43)$ and I-A $(N=17)$ Groups by Measurement Time

Note: Error bars are \pm 1 S.E.; S-I = Stable-Inactive, I-A = Inactive-Active; ${ }^{\text {a }} \mathrm{S}-\mathrm{I}>\mathrm{I}-\mathrm{A}$ Main Effect $(\mathrm{p}<.05)$ 


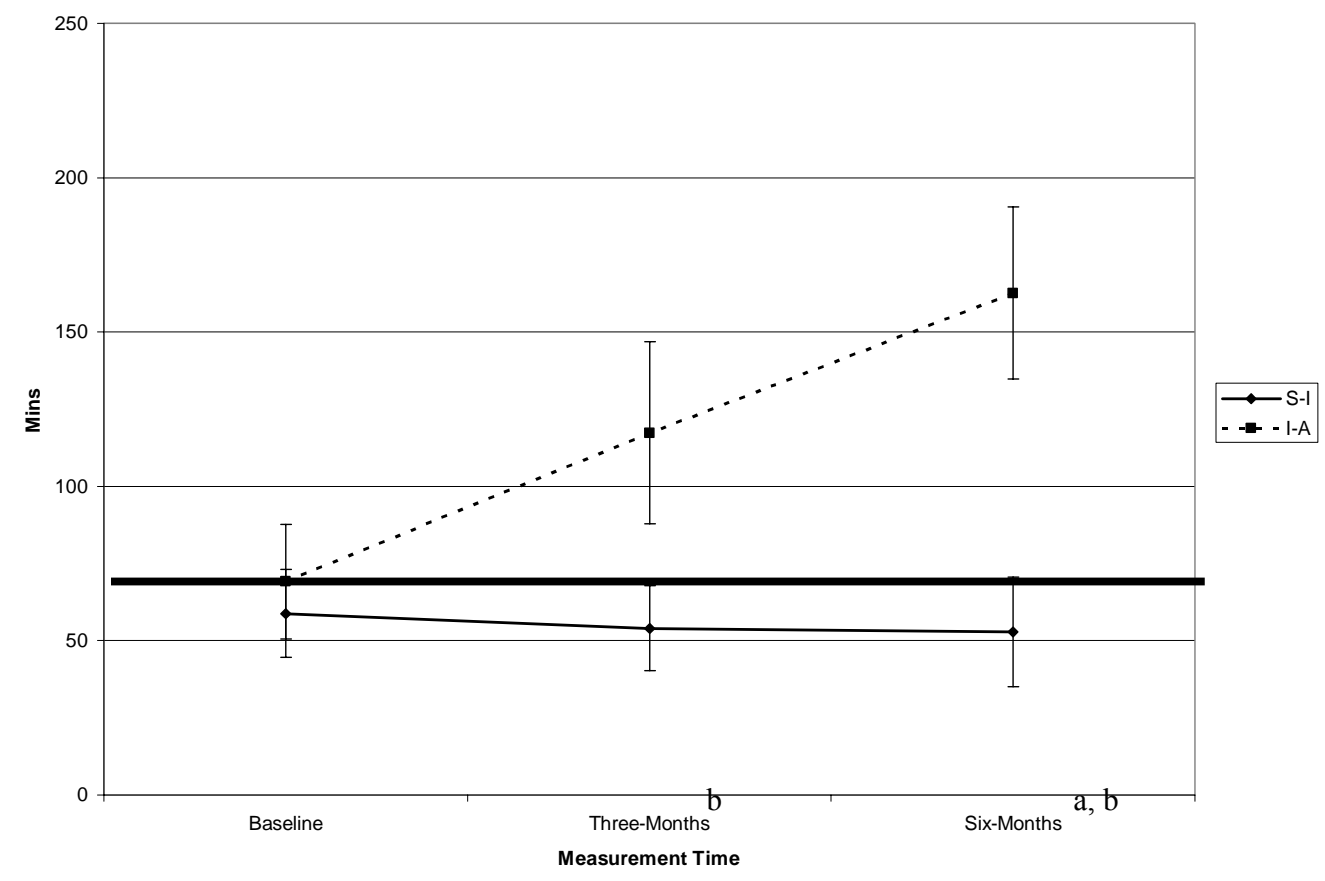

Figure 4.7 Mean Total Minutes of Exercise for S-I ( $N=43)$ and I-A $(N=17)$ Groups by Measurement Time

Note: Error bars are \pm 1 S.E.; S-I $=$ Stable-Inactive, I-A = Inactive-Active; ${ }^{a}$ I-A $>$ S-I at Six Months $(\mathrm{p}<.05){ }^{\mathrm{b}}$ Six Months $<$ Three Months for I-A group $(\mathrm{p}<.05)$; Dark horizontal line indicates 60 minutes per week of total exercise, or the minimum criteria for "active" status as set in this study. 


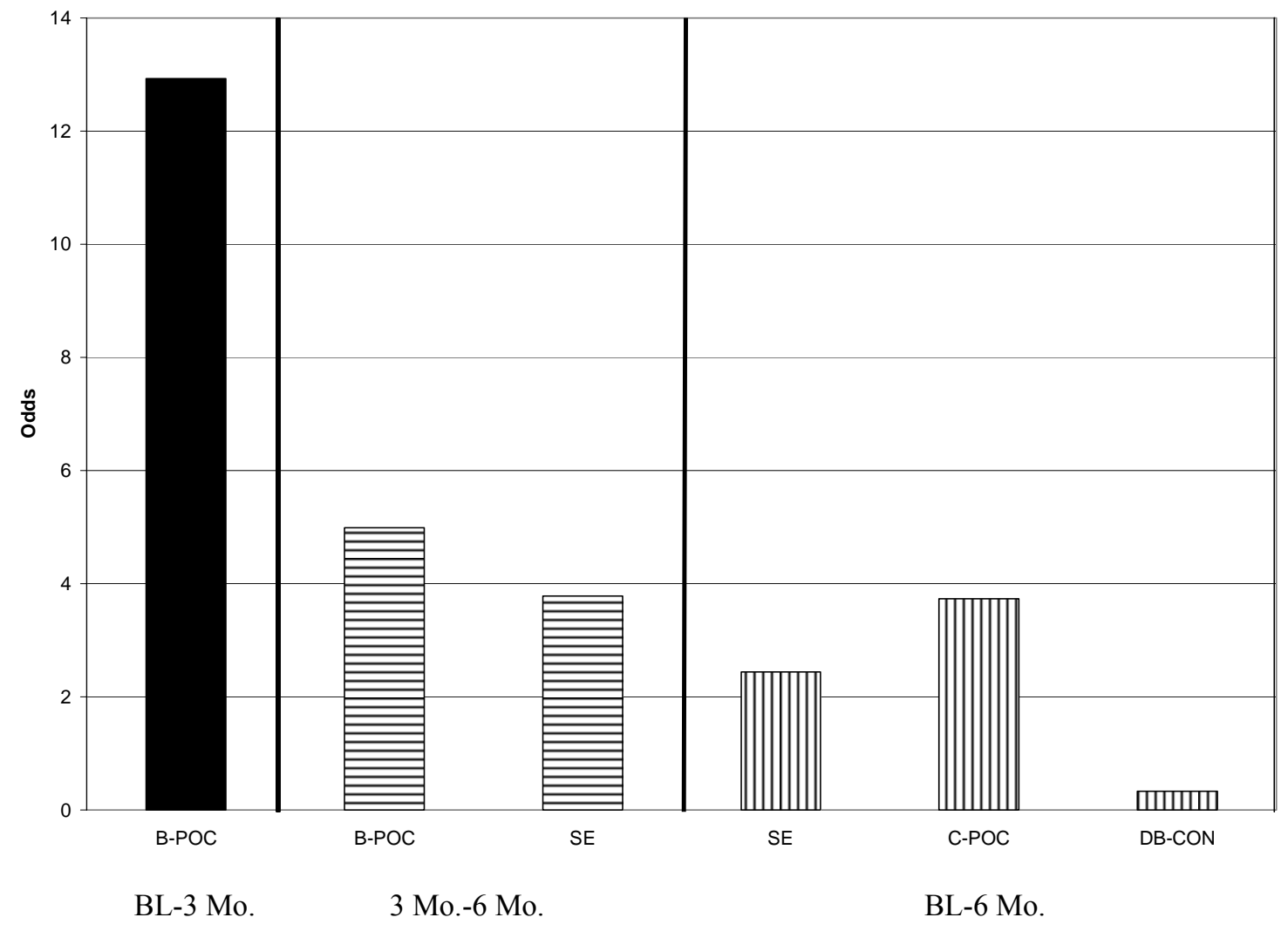

Figure 4.8 Odds Ratios for TTM Constructs Retained in Multivariate Logistic Regression Models by Time Frame

Note: $\mathrm{B}-\mathrm{POC}=$ Behavioral Processes of Change; $\mathrm{SE}=$ Self-efficacy; $\mathrm{C}-\mathrm{POC}=$ Cognitive Processes of Change; DB-CON=Decisional Balance-Cons 


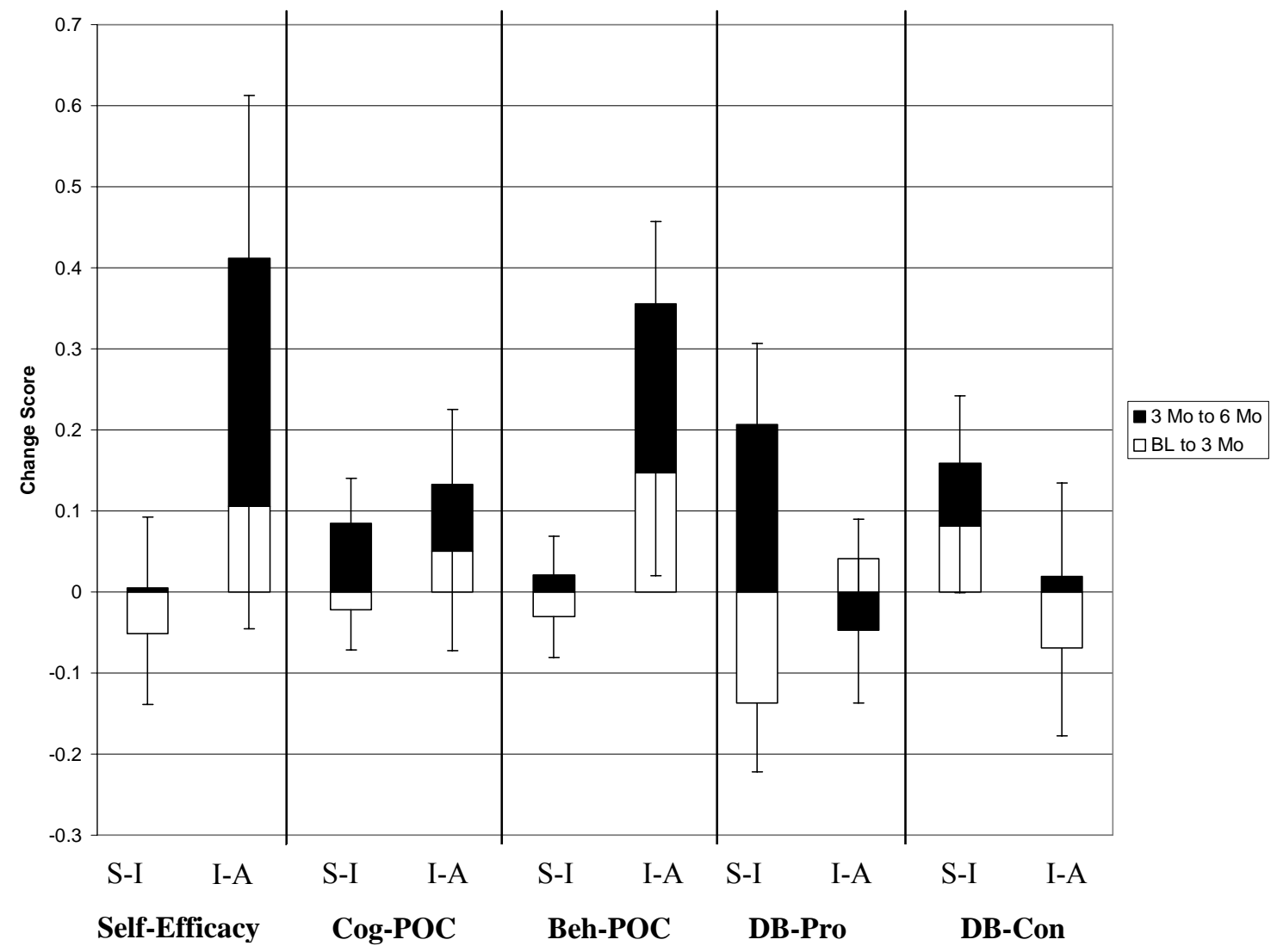

Figure 4.9 Mean Change Scores for TTM Constructs Showing Contribution of Measurement Times

Note: Error bars are \pm 1 S.E.; S-I = Stable-Inactive, I-A = Inactive-Active; Cog$\mathrm{POC}=$ Cognitive Processes of Change; Beh-POC $=$ Behavioral Processes of Change; DBPro=Decisional Balance-Pros; DB-Con=Decisional Balance-Cons 


\section{REFERENCES}

ACSM. (1990). American College of Sports Medicine position stand. The recommended quantity and quality of exercise for developing and maintaining cardiorespiratory and muscular fitness in healthy adults. Medicine and Science in Sports and Exercise, 22(2), 265-274.

ACSM. (1998). American College of Sports Medicine Position Stand: The recommended quantity and quality of exercise for developing and maintaining cardiorespiratory and muscular fitness, and flexibility in healthy adults. Medicine and Science in Sports and Exercise, 30, 975-991.

ACSM. (2000). ACSM's guidelines for exercise testing and prescription. (6th ed.). Baltimore: Lippincott, Williams, and Wilkins.

Adams, J., \& White, M. (2003). Are activity promotion interventions based on the transtheoretical model effective? A critical review. British Journal of Sports Medicine, 37, 106-114.

Ajzen, I. (1991). The theory of planned behavior. Organizational Behavior and Human Decision Processes, 50, 179-211.

Ajzen, I., \& Madden, T. J. (1986). Prediction of goal-directed behavior: Attitudes, intentions, and perceived behavioral control. Journal of Experimental Social Psychology, 22, 453-474.

Armstrong, C. A., Sallis, J. F., Hovell, M. F., \& Hofstetter, C. R. (1993). Stages of change, self-efficacy, and the adoption of vigorous exercise: A prospective analysis. Journal of Sport \& Exercise Psychology, 15(4), 390-402.

Bandura, A. (1991). Social cognitive theory of self-regulation. Organizational Behavior and Human Decision Processes, 50, 248-287.

Bandura, A. (1997). The anatomy of stages of change. American Journal of Health Promotion, 12(1), 8-10. 
Baranowski, T., Anderson, C., \& Carmack, C. (1998). Mediating variable framework in physical activity interventions: How are we doing? How might we do better? American Journal of Preventive Medicine, 15(4), 266-297.

Blair, S. N., Haskell, W. L., Ho, P., Paffenbarger, R. S., Vranizan, K. M., Farquhar, J. W., et al. (1985). Assessment of habitual physical activity by a seven-day recall in a community survey and controlled experiments. American Journal of Epidemiology, 122(5), 794-804.

Blissmer, B., \& McAuley, E. (2002). Testing the requirements of stages of physical activity among adults: The comparative effectiveness of stage-matched, mismatched, standard care, and control interventions. Annals of Behavioral Medicine, 24(3), 181-189.

Blue, C. L., Black, D. R., Conrad, K., \& Gretebeck, K. A. (2003). Beliefs of blue-collar workers: Stage of readiness for exercise. American Journal of Health Behavior, 27(4), 408-420.

Bock, B. C., Marcus, B. H., Pinto, B. M., \& Forsyth, L. H. (2001). Maintenance of physical activity following an individualized motivationally tailored intervention. Annals of Behavioral Medicine : a Publication of the Society of Behavioral Medicine, 23(2), 79-87.

Buckworth, J., \& Dishman, R. K. (2002). Exercise Psychology. Champaign, IL: Human Kinetics.

Buckworth, J., \& Wallace, L. S. (2002). Application of the Transtheoretical Model to physically active adults. The Journal of Sports Medicine and Physical Fitness, 42(3), 360-367.

Bull, S. S., Gillette, C., Glasgow, R. E., \& Estabrooks, P. A. (2003). Work site health promotion research: to what extent can we generalize the results and what is needed to translate research to practice? Health Education \& Behavior : the Official Publication of the Society for Public Health Education, 30(5), 537-549.

Cardinal, B. J. (1997a). Construct validity of stages of change for exercise behavior. American Journal of Health Promotion : AJHP, 12(1), 68-74.

Cardinal, B. J. (1997b). Predicting exercise behavior using components of the transtheoretical model of behavior change. Journal of Sport Behavior, 20(3), 272283.

Cardinal, B. J., Kosma, M., \& McCubbin, J. A. (2004). Factors influencing the exercise behavior of adults with physical disabilities. Medicine and Science in Sports and Exercise, 36(5), 868-875. 
Cardinal, B. J., Tuominen, K. J., \& Rintala, P. (2003). Psychometric assessment of Finnish versions of exercise-related measures of Transtheoretical Model constructs. International Journal of Behavioral Medicine, 10(1), 31-43.

Carmack-Taylor, C. L., Boudreaux, E. D., Jeffries, S. K., Scarinci, I. C., \& Brantley, P. J. (2003). Applying exercise stage of change to a low-income underserved population. American Journal of Health Behavior, 27(2), 99-107.

CDC. (2004). Behavioral Risk Factor Surveillance System Survey Questionnaire. Atlanta, Georgia: U.S. Department of Health and Human Services, Centers for Disease Control and Prevention.

CDC. (2000). Behavioral Risk Factor Surveillance System.

Dallow, C. B., \& Anderson, J. (2003). Using self-efficacy and a transtheoretical model to develop a physical activity intervention for obese women. American Journal of Health Promotion, 17(6), 373-381.

Dannecker, E. A., Hausenblas, H. A., Connaughton, D. P., \& Lovins, T. R. (2003). Validation of a stages of exercise change questionnaire. Research Quarterly for Exercise and Sport, 74(3), 236-247.

Dillman, D. A. (2000). Mail and internet surveys: The tailored design method. New York: John Wiley.

Dishman, R. K. (1988). Exercise adherence research: Future directions. American Journal of Health Promotion, 3, 52-56.

Dishman, R. K. (2001). The problem of exercise adherence: Fighting sloth in nations with market economies. Quest, 53, 279-294.

Dunn, A. L., Garcia, M. E., Marcus, B. H., Kampert, J. B., Kohl, H. W., \& Blair, S. N. (1998). Six month physical activity and fitness changes in Project Active, a randomized trial. Medicine and Science in Sports and Exercise, 30(7), 1076-1083.

Fahrenwald, N. L., \& Walker, S. N. (2003). Application of the Transtheoretical Model of Behavior Change to the physical activity of WIC mothers. Public Health Nursing, 20(4), 307-317.

Fink, A., \& Kosecoff, J. (1998). How to conduct surveys. Thousand Oaks, CA: Sage.

Fishbein, M. (1967). Readings in attitude theory and measurement. New York: Wiley. 
Fishbein, M., \& Ajzen, I. (1975). Belief, attitude, intention, and behavior: An introduction to theory and research. Reading, MA: Addison-Wesley.

Fowler, F. J. (2002). Survey Research Methods (Third Edition ed.). Thousand Oaks, CA: Sage.

Godin, G., \& Shepard, R. J. (1985). A simple method to assess exercise behavior in the community. Canadian Journal of Applied Sport Sciences, 10, 141-146.

Gorely, T., \& Gordon, S. (1995). An examination of the transtheoretical model and exercise behavior in older adults. Journal of Sport \& Exercise Psychology, 17(3), 312-324.

Herrick, A. B., Stone, W. J., \& Mettler, M. M. (1997). Stages of change, decisional balance, and self-efficacy across four health behaviors in a worksite environment. American Journal of Health Promotion, 12(1), 49-56.

Higgins, L. C., \& Oldenburg, B. (2003). Predictors of progression and regression in exercise adoption in young women. Journal of Applied Social Psychology, 33(4), 716-729.

Hosmer, D. W., \& Lemeshow, S. (2000). Applied logistic regression (2nd ed.). New York: John Wiley \& Sons, Inc.

Janz, N. K., \& Becker, M. H. (1984). The health belief model: A decade later. Health Education Quarterly, 11, 1-47.

Jordan, P. J., Nigg, C. R., Norman, G. J., Rossi, J. S., \& Benisovich, S. V. (2002). Does the transtheoretical model need an attitude adjustment? Integrating attitude with decisional balance as predictors of stage of change for exercise. Psychology of Sport \& Exercise, 3(1), 65-83.

Kearney, J. T., Stull, G. A., Ewing, J. L., Jr., \& Strein, J. W. (1976). Cardiorespiratory responses of sedentary college women as a function of training intensity. Journal of Applied Physiology, 41(6), 822-825.

Keppel, G. (1991). Design and analysis: A researcher's handbook. (3rd ed.). Upper Saddle River, NJ: Prentice Hall.

Kosma, M., Cardinal, B. J., \& McCubbin, J. A. (2004). Predictors of physical activity stage of change among adults with physical disabilities. American Journal of Health Promotion, 19(2), 114-117.

Kosma, M., Cardinal, B. J., \& McCubbin, J. A. (2005). A pilot study of a web-based physical activity motivational program for adults with physical disabilities. Disability and Rehabilitation, 27(23), 1435-1442. 
Kosma, M., Gardner, R. E., Cardinal, B. J., Bauer, J. J., \& McCubbin, J. A. (2006). Psychosocial determinants of stages of change and physical activity among adults with physical disabilities. Adapted Physical Activity Quarterly, 23(1), 49-64.

Leslie, E., Johnson-Kozlow, M., Sallis, J. F., Owen, N., \& Bauman, A. (2003). Reliability of moderate-intensity and vigorous physical activity stage of change measures for young adults. Preventive Medicine, 37, 177-181.

Lewis, B. A., Forsyth, L. H., Pinto, B. M., Bock, B. C., Roberts, M. B., \& Marcus, B. H. (2006). Psychosocial mediators of physical activity in a randomized controlled intervention trial. Journal of Sport \& Exercise Psychology, 28(2), 193-204.

Lippke, S., \& Ziegelmann, J. P. (2006). Understanding and modeling health behavior: The multi-stage model of health behavior change. Journal of Health Psychology, 11(1), 37-50.

Lippke, S., Ziegelmann, J. P., \& Schwarzer, R. (2004). Initiation and maintenance of physical exercise: Stage-specific effects of a planning intervention. Research in Sports Medicine., 12, 1-20.

Loughlan, C., \& Mutrie, N. (1997). An evaluation of the effectiveness of three interventions in promoting physical activity in a sedentary population. Health Education Journal, 56(154-165).

Maddison, R., \& Prapavessis, H. (2006). Exercise behavior among New Zealand adolescents: A test of the Transtheoretical Model. Pediatric Exercise Science, 18(3), 351-363.

Marcus, B. H., Banspach, S. W., Lefebvre, R. C., Rossi, J. S., Carleton, R. A., \& Abrams, D. B. (1992a). Using the stages of change model to increase the adoption of physical activity among community participants. American Journal of Health Promotion, 6(6), 424-429.

Marcus, B. H., Banspach, S. W., Lefebvre, R. C., Rossi, J. S., Carleton, R. A., \& Abrams, D. B. (1992b). Using the stages of change model to increase the adoption of physical activity among community participants. American Journal of Health Promotion : AJHP, 6(6), 424-429.

Marcus, B. H., Bock, B. C., Pinto, B. M., Forsyth, L. H., Roberts, M. B., \& Traficante, R. M. (1998). Efficacy of an individualized, motivationally-tailored physical activity intervention. Annals of Behavioral Medicine, 20(3), 174-180.

Marcus, B. H., Rakowski, W., \& Rossi, J. S. (1992). Assessing motivational readiness and decision making for exercise. Health Psychology, 11(4), 257-261. 
Marcus, B. H., Rossi, J. S., Selby, V. C., Niaura, R. S., \& Abrams, D. B. (1992). The stages and processes of exercise adoption and maintenance in a worksite sample. Health Psychology, 11(6), 386-395.

Marcus, B. H., Selby, V. C., Niaura, R. S., \& Rossi, J. S. (1992). Self-efficacy and the stages of exercise behavior change. Research Quarterly for Exercise and Sport, 63(1), 60-66.

Marcus, B. H., \& Simkin, L. R. (1993). The stages of exercise behavior. Journal of Sports Medicine and Physical Fitness, 33, 83-88.

Marcus, B. H., Simkin, L. R., Rossi, J. S., \& Pinto, B. M. (1996). Longitudinal shifts in employees' stages and processes of exercise behavior change. American Journal of Health Promotion, 10(3), 195-200.

Marshall, S. J., \& Biddle, S. J. (2001). The transtheoretical model of behavior change: A meta-analysis of applications to physical activity and exercise. Annals of Behavioral Medicine, 23(4), 229-246.

Marttila, J., \& Nupponen, R. (2003). Assessing stage of change for physical activity: How congruent are parallel methods? Health Education Research, 18(4), 419428.

Matthews, C. E., Freedson, P. S., Hebert, J. R., Stanek, E. J., Merriam, P. A., Rosal, M. C., et al. (2001). Seasonal variation in household, occupational, and leisure time physical activity: Longitudinal analyses from the seasonal variation of blood cholesterol study. American Journal of Epidemiology, 153(2), 172-183.

McMillan, J. H., \& Schumacher, S. (1997). Research in Education: A Conceptual Introduction (Fourth Edition ed.). New York: Longman.

Miilunpalo, S., Nupponen, R., Laitakari, J., Marttila, J., \& Paronen, O. (2000). Stages of change in two modes of health-enhancing physical activity: methodological aspects and promotional implications. Health Education Research, 15(4), 435448.

Naylor, P. J., Simmonds, G., Riddoch, C., Velleman, G., \& Turton, P. (1999).

Comparison of stage-matched and unmatched interventions to promote exercise behaviour in the primary care setting. Health Education Research, 14(5), 653666.

Nichols, J. F., Wellman, E., Caparosa, S., Sallis, J. F., Calfas, K. J., \& Rowe, R. (2000). Impact of a worksite behavioral skills intervention. American Journal of Health Promotion, 14(4), 218-221. 
Nigg, C. R. (2001). Explaining adolescent exercise behavior change: a longitudinal application of the transtheoretical model. Annals of behavioral medicine : a publication of the Society of Behavioral Medicine, 23(1), 11-20.

Nigg, C. R. (2005). There is more to stage of exercise than just exercise. Exercise and Sport Sciences Reviews, 33(1), 32-35.

Nigg, C. R., \& Courneya, K. S. (1998). Transtheoretical model: Examining adolescent exercise behavior. Journal of Adolescent Health, 22(3), 214-224.

Norman, G. J. (1998). A cluster analytic test of the transtheoretical model applied to exercise behavior., University of Rhode Island.

O'Hea, E. L., Boudreaux, E. D., Jeffries, S. K., Taylor, C. L. C., Scarinci, I. C., \& Brantley, P. J. (2004). Stage of change movement across three health behaviors: The role of self-efficacy. American Journal of Health Promotion, 19(2), 94-102.

O'Hea, E. L., Wood, K. B., \& Brantley, P. J. (2003). The transtheoretical model: gender differences across 3 health behaviors. American Journal of Health Behavior, 27(6), 645-656.

Oldenburg, B., Glanz, K., \& Ffrench, M. (1999). The application of staging models to the understanding of health behaviour change and the promotion of health. Psychology \& Health, 14(3), 503-516.

Pate, R. R., Pratt, M., Blair, S. N., Haskell, W. L., Macera, C. A., Bouchard, C., et al. (1995). Physical activity and public health. A recommendation from the Centers for Disease Control and Prevention and the American College of Sports Medicine. JAMA : the Journal of the American Medical Association, 273(5), 402-407.

Petosa, S. (1995). Use of social cognitive theory to explain exercise behavior among adults., The Ohio State University, Columbus.

Pinto, B. M., Lynn, H., Marcus, B. H., DePue, J., \& Goldstein, M. G. (2001). Physicianbased activity counseling: intervention effects on mediators of motivational readiness for physical activity. Annals of Behavioral Medicine : a Publication of the Society of Behavioral Medicine, 23(1), 2-10.

Pinto, B. M., \& Marcus, B. H. (1995). A stages of change approach to understanding college students' physical activity. Journal of American College Health, 44(1), 27-31.

Plotnikoff, R. C., Hotz, S. B., Birkett, N. J., \& Courneya, K. S. (2001). Exercise and the transtheoretical model: a longitudinal test of a population sample. Preventive Medicine, 33(5), 441-452. 
Pollock, M. L., Gaesser, G. A., Butcher, J. D., Després, J.-P., Dishman, R. K., Franklin, B. A., et al. (1998). American College of Sports Medicine position stand. The recommended quantity and quality of exercise for developing and maintaining cardiorespiratory and muscular fitness, and flexibility in healthy adults. Medicine and Science in Sports and Exercise, 30(6), 975-991.

Prochaska, J. O. (2006). Moving beyond the transtheoretical model. Addiction, 101(6), 768-774.

Prochaska, J. O., \& DiClemente, C. C. (1983). Stages and processes of self-change in smoking: Towards an integrative model of change. Journal of Counseling and Clinical Psychology, 51, 390-395.

Prochaska, J. O., \& DiClemente, C. C. (1992). In search of how people change: Applications to addictive behaviors. American Psychologist, 47(9), 1102-1114.

Prochaska, J. O., Redding, C. A., \& Evers, K. E. (2002). The transtheoretical model and stages of change. In K. Glanz, B. K. Rimer \& F. M. Lewis (Eds.), Health behavior and health education: Theory, research, and practice. (3rd ed.). San Francisco: Jossey-Bass.

Prochaska, J. O., \& Velicer, W. F. (1997a). Misinterpretations and misapplications of the transtheoretical model. American Journal of Health Promotion, 12(1), 11-12.

Prochaska, J. O., \& Velicer, W. F. (1997b). The transtheoretical model of health behavior change. American Journal of Health Promotion, 12(1), 38-48.

Prochaska, J. O., Velicer, W. F., DiClemente, C. C., \& Fava, J. (1988). Measuring processes of change: Applications to the cessation of smoking. Journal of Consulting and Clinical Psychology, 56(4), 520-528.

Prochaska, J. O., Velicer, W. F., Rossi, J. S., Goldstein, M. G., Marcus, B. H., Rakowski, W., et al. (1994). Stages of change and decisional balance for 12 problem behaviors. Health Psychology, 13(1), 39-46.

Reed, G. R., Velicer, W. F., Prochaska, J. O., Rossi, J. S., \& Marcus, B. H. (1997). What makes a good staging algorithm: Examples from regular exercise. American Journal of Health Promotion, 12, 57-66.

Resnick, B., \& Nigg, C. R. (2003). Testing A Theoretical Model of Exercise Behavior for Older Adults. Nursing Research, 52(2), 80-88.

Rhodes, R. E., \& Plotnikoff, R. C. (2006). Understanding Action Control: Predicting Physical Activity Intention-Behavior Profiles Across 6 Months in a Canadian Sample. Health Psychology, 25(3), 292-299. 
Rodgers, W. M., Courneya, K. S., \& Bayduza, A. L. (2001). Examination of the transtheoretical model and exercise in 3 populations. American Journal of Health Behavior, 25(1), 33-41.

Rosen, C. S. (2000). Is the sequencing of change processes by stage consistent across health problems? A meta-analysis. Health psychology: official journal of the Division of Health Psychology American Psychological Association, 19(6), 593 604.

Sallis, J. F., \& Owen, N. (1999). Physical Activity and Behavioral Medicine. Thousand Oaks, CA: Sage.

Sarkin, J. A., Johnson, S. S., Prochaska, J. O., \& Prochaska, J. M. (2001). Applying the transtheoretical model to regular moderate exercise in an overweight population: validation of a stages of change measure. Preventive Medicine, 33(5), 462-469.

Schumann, A., Estabrooks, P. A., Nigg, C. R., \& Hill, J. (2003). Validation of the stages of change with mild, moderate, and strenuous physical activity behavior, intentions, and self-efficacy. International Journal of Sports Medicine, 24, 363365.

Schumann, A., Nigg, C. R., Rossi, J. S., Jordan, P. J., Norman, G. J., Garber, C. E., et al. (2002). Construct validity of the stages of change of exercise adoption for different intensities of physical activity in four samples of differing age. American Journal of Health Promotion, 16, 280-287.

Schwarzer, R. (1992). Self-efficacy in the adoption and maintenance of health behaviours: Theoretical approaches and a new model. In R. Schwarzer (Ed.), Selfefficacy: Thought control of action. (pp. 217-243). Washington, DC: Hemisphere Publishing Corporation.

U.S. Department of Health and Human Services. (1996). Physical activity and health: A report of the Surgeon General. Atlanta, GA: Centers for Disease Control and Prevention. National Center for Chronic Disease Prevention and Health Promotion.

U.S. Department of Health and Human Services. (2000). Healthy People 2010. Washington D.C.: U.S. Department of Health and Human Services.

Spencer, L., Adams, T. B., Malone, S., Roy, L., \& Yost, E. (2006). Applying the Transtheoretical Model to Exercise: A Systematic and Comprehensive Review of the Literature. Health Promotion Practice, 7(4), 428-443.

Sutton, S. R. (2000). Interpreting cross-sectional data on stages of change. Psychology and Health, 15, 163-171. 
Sutton, S. R. (2001). Back to the drawing board? A review of applications of the transtheoretical model to substance use. Addiction, 96, 175-186.

Tung, W. C., Gillett, P. A., \& Pattillo, R. E. (2005). Applying the transtheoretical model to physical activity in family caregivers in Taiwan. Public Health Nursing, 22(4), 299-310.

Wallace, L. S., \& Buckworth, J. (2003). Longitudinal shifts in exercise stages of change in college students. Journal of Sports Medicine and Physical Fitness, 43, 209212.

Weinstein, N. D. (1988). The precaution adoption process model. Health Psychology, 7, 355-386.

Weinstein, N. D. (2007). Misleading tests of health behavior theories. Annals of Behavioral Medicine, 33(1), 1-10.

Weinstein, N. D., Rothman, A. J., \& Sutton, S. R. (1998). Stage theories of health behavior: Conceptual and methodological issues. Health Psychology, 17(3), 290299.

West, R. (2005). Time for a change: Putting the Transtheoretical (Stages of Change) Model to rest. Addiction, 100, 1036-1039.

Woods, C., Mutrie, N., \& Scott, M. (2002). Physical activity intervention: a transtheoretical model-based intervention designed to help sedentary young adults become active. Health education research, 17(4), 451-460.

Wyse, J., Mercer, T., Ashford, B., Buxton, K., \& Gleeson, N. (1995). Evidence for the validity and utility of the Stages of Exercise Behaviour Change Scale in young adults. Health Education Research, 10(3), 365-377. 
APPENDIX A

WORKSITE INVITATION LETTER AND FACT SHEET 
1 February, 2006

\section{Dear Sir/Madam:}

This is letter is an overview of my $\mathrm{PhD}$ research project, the Worksite Exercise Behavior Study (WEBS). I plan to conduct a 6-month study of physical activity patterns among working adults in Ohio. My goal is to examine how working adults go from being sedentary to adopting a physically active lifestyle. I hope that I can recruit volunteers from your company during the winter of 2005-06 for this study.

I'm sure you already know that physically fit workers have fewer disability days, and reduced workers' compensation claims. In addition to the health benefits of physical activity and the potential for reducing risk of chronic disease and accidents, studies show that active employees report feeling less tired, more relaxed, more self-confident, and more productive.

Let me tell you a little more about the study. I would like to randomly select employees from your worksite (from a complete employee list) to participate in the study. The questionnaire will be completed through the internet, and will take 15-20 minutes. Volunteers will be contacted to complete the questionnaire again 3- and 6-months later. Employee information will remain confidential. No employee names will be collected. If you want to preview the full questionnaire, please visit the following URL: http://education.osu.edu/jbuckworth/exercisesurvey/test.htm (username: exercisesurvey, password: exercise). As a benefit of participation in the study, I would like to offer two workshops for your employees on tips for starting and sticking with exercise, and some different ways they can fit exercise into their busy lives. These workshops are optional, and can be scheduled at your convenience. For more information about the study, please see the attached WEBS summary page.

If you have questions and/or are willing to make this opportunity available to your employees, please contact me, Christopher Fink at The Ohio State University, at fink.93@osu.edu or 614-570-6013. If I have not already heard from you, I will be following up in one week to see if you'll let me conduct this research project with your company. 
This research project is sponsored by the School of Physical Activity and Educational Services at The Ohio State University, and the prinicpal investigator is Dr. Janet Buckworth, my dissertation advisor. I look forward to hearing from you.

Thank you for your consideration.

Christopher L. Fink, M.S.

Ph.D. Candidate

The Ohio State University

340 Cunz Hall

1841 Millikin Rd.

Columbus, $\mathrm{OH} 43210$
Janet Buckworth, Ph.D., FACSM

Associate Professor

The Ohio State University

169 Cunz Hall

1841 Millikin Rd.

Columbus, $\mathrm{OH} 43210$ 


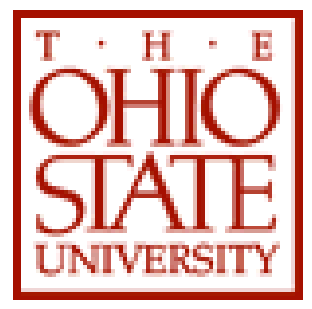

\section{WEBS (The Worksite Exercise Behavior Study)}

The following is a summary of important information about WEBS:

\section{WEBS Details}

- Employees randomly selected from a full employee list

- Study will use an online questionnaire

0 View questionnaire at http://education.osu.edu/jbuckworth/exercisesurvey/test.htm

- Username: exercisesurvey

- Password: exercise

o No employee names or personally identifiable information will be collected

o No IP addresses will be stored

- No information related to place of employment will be stored

o Data will NOT be analyzed in the context of employer or company

- Study data will be made available to volunteers for one month after study

- Seminars will be offered after the study on ways to fit exercise into a busy life

\section{Volunteer Commitment}

- Potential volunteers will be contacted a maximum of three times via email and office mail to participate in the study

o If no reply is received, no further contact will be made

- Volunteers will average 20 minutes for completion of an online questionnaire

- Volunteers will complete the questionnaire three times over a 6 month span

o At the start of the study, 3-, and 6-months later

- Total time commitment for the 6 months will be 1 hour

- Study data will be made available to volunteers for one month after completion 


\section{Company \& Employee Benefits}

- Access to information (after the study) about employee exercise patterns and beliefs

o Data will NOT be specific to each company

- Enhances atmosphere of concern for employee well-being

- Two seminars offered to employees on ways to fit exercise into a busy life

- Supporting research on employee health habits 
APPENDIX B

IRB APPROVAL 


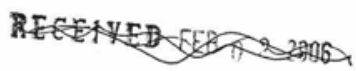

TITLE PAGE - APPLICATION FOR EXEMPTION For office use only FROM REVIEW BY THE INSTITUTIONAL REVIEW BOARD The Ohio State University, Columbus OH 43210

PROTOCOL NUMBER: 20060090

\begin{tabular}{|c|c|c|}
\hline$\Delta$ Principal Investigator & Name: Janet Buckworth & Phone: $614-292-0757$ \\
\hline \multirow{3}{*}{$\begin{array}{l}\text { University Title: } \\
\begin{array}{|l}\square \text { Professor } \\
\square \text { Associate Professor } \\
\square \text { Assistant Professor } \\
\square \text { Instructor } \\
\square \text { Other. Please specify. } \\
\text { (May require prior approval.) }\end{array}\end{array}$} & Department or College: EDU PAES & \multirow[t]{2}{*}{$\begin{array}{l}\text { E-mail: } \\
\text { buckworth.1@osu.edu }\end{array}$} \\
\hline & $\begin{array}{l}\text { Campus Address (room, building, street address): } \\
169 \text { Cunz Hall } \\
1841 \text { Milliken Rd. } \\
\text { Columbus, OH } 43210\end{array}$ & \\
\hline & Toate: & Fax: $\quad 614-688-3432$ \\
\hline \multirow{3}{*}{$\begin{array}{l}\text { Co-Investigator } \\
\text { University Status: } \\
\square \text { Faculty } \\
\square \text { Staff } \\
\text { Q Graduate Student } \\
\square \text { Undergraduate Student } \\
\square \text { Other. Please specify. }\end{array}$} & Name: Christopher Fink & Phone: $614-570-6013$ \\
\hline & $\begin{array}{l}\text { Campus Address (room, building, street address) or } \\
\text { Mailing Address: } \\
155 \text { Cunz Hall } \\
1841 \text { Milliken Rd. } \\
\text { Columbus, OH } 43210\end{array}$ & E-mail: fink.93@osu.edu \\
\hline & Date: & Fax: $\quad 614-688-3432$ \\
\hline \multirow{3}{*}{$\begin{array}{l}\text { Do-Investigator } \\
\text { University Status: } \\
\square \text { Faculty } \\
\square \text { Staff } \\
\square \text { Graduate Student } \\
\square \text { Undergraduate Student } \\
\square \text { Other. Please specify. }\end{array}$} & Name: & Phone: \\
\hline & $\begin{array}{l}\text { Campus Address (room, building, street address) or } \\
\text { Mailing Address: }\end{array}$ & E-mail: \\
\hline & Signature: & Fax: \\
\hline
\end{tabular}

\begin{tabular}{|l|l|}
\hline Protocol Title & A worksite examination of the transtheoretical model in exercise \\
\hline
\end{tabular}

\begin{tabular}{|l|l|}
\hline Source of Funding & n/a \\
\hline
\end{tabular}

\begin{tabular}{|c|c|c|}
\hline $\begin{array}{l}\text { For Office Use Only } \\
\text { A Approved. }\end{array}$ & $\checkmark$ & $\begin{array}{l}\text { Research has been determined to be exempt under these categories: } \\
\text { Research may begin as of the date of determination listed below. }\end{array}$ \\
\hline$\square$ Disapproved. & $\nabla$ & $\begin{array}{l}\text { The proposed research does not fall within the categories of exemption. Submit an application } \\
\text { to the appropriate Institutional Review Board for review. }\end{array}$ \\
\hline of determir & & Ofone A. Schu \\
\hline
\end{tabular}

HSE-1.0

REC'D FEB 012006

Page 2

Approved by the Policy Coordinating IRB, 5/1800, revised 01/09/06 
APPENDIX C

WORKSITE APPROVAL LETTERS 


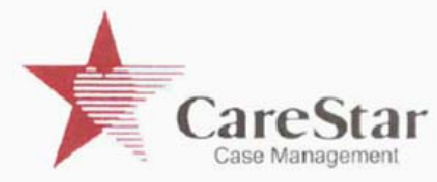

February 7,2006

Janet Buckworth, Ph.D., FACSM

School of Physical Activity and Educational Services

College of Education

169 Cunz Hall

1841 Millikin Road

Columbus, $\mathrm{OH} 43210$

Dear Dr. Buckworth:

I have reviewed the dissertation research proposal from Christopher Fink which requests a sample of employees from Care Star for participation in the survey research he is proposing. We approve the research request under the terms of the proposal.

I am providing this letter in support of the application for review by the Internal Review Board (IRB) of the Office of Responsible Research Practices. Once the IRB approval is granted, we will provide required sample of Care Star employees for your research.

Sincerely,

Tally Fraarman

Kathleen M. Staarmann

Human Resources Director

\begin{tabular}{|c|c|c|c|c|}
\hline $\begin{array}{l}\text { Cincinnati } \\
5562 \text { Cheviot Road } \\
\text { Cincinnati, Ohio } 45247 \\
\text { Oifice } 513-618-8300 \\
\text { Toll Free 866-834-4712 } \\
\text { Fax 513-618-8319 }\end{array}$ & $\begin{array}{l}\text { Columbus } \\
6100 \text { Channingway Blvo. } \\
\text { Suite } 700 \\
\text { Columbus, Ohio } 43232 \\
\text { Office } 614-751-7777 \\
\text { Toll Free } 800-616-3718 \\
\text { Fax } 614-751-7770\end{array}$ & $\begin{array}{l}\text { Canton } \\
4450 \text { Balden Village Street } \\
\text { Suite } 504 \\
\text { Canton, } 0 \text { hio } 44718 \\
\text { Office } 330-492-4530 \\
\text { Toll Free } 800-423-1046 \\
\text { Fax } 330-492-0776\end{array}$ & $\begin{array}{l}\text { Cleveland } \\
4141 \text { Rockside Road } \\
\text { Suite } 210 \\
\text { Seven Hils, Onio } 44131 \\
\text { Office } 216-532-2000 \\
\text { Toll Free } 800-442-1857 \\
\text { Fax 216-532-2001 }\end{array}$ & $\begin{array}{l}\text { Dayton } \\
\text { 81-A Ahoads Center Drive } \\
\text { Centerville, Ohio } 45458 \\
\text { Ottice } 937-435-1161 \\
\text { Toll Free } 800-538-4218 \\
\text { Fax } 937-435-0980 \\
\text { www.carestar.com }\end{array}$ \\
\hline
\end{tabular}


March 7, 2006

Janet Buckworth, Ph.D., FACSM

School of Physical Activity and Educational Services

College of Education

169 Cunz Hall

1841 Millikin Road

Columbus, $\mathrm{OH} 43210$

Dear Dr. Buckworth:

Our office has reviewed the dissertation research proposal from Christopher Fink which requests a sample of employees from American Electric Power (AEP) for participation in the survey research he is proposing. We approve the research request under the terms of the proposal.

I am providing this letter in support of the application for review by the Internal Review Board (IRB) of the Office of Responsible Research Practices. Once the IRB approval is granted, we will provide the required sample of AEP employees for your research.

Sincerely,

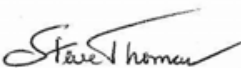

Stephen D. Thomas

HR Manager Corporate

c: Christopher Fink

AEP:America's Energy Partner ${ }^{\circledR}$ 
Phone (614) 292-4164

January 13,2006

Janet Buckworth, Ph.D., FACSM

School of Physical Activity and Educational Services

College of Education

169 Cunz Hall

1841 Millikin Road

Columbus, $\mathrm{OH} 43210$

Dear Dr. Buckworth:

I have reviewed the request for the Office of Human Resources to assist Christopher Fink, a graduate student in your department, in his dissertation research by providing a sample of faculty and staff from the Human Resources system and have approved the request. Provision of such a sample is permitted for university research purposes.

I provide this letter to you in support of your application for review by the Internal Review Board (IRB) of the Office of Responsible Research Practices. Once you have IRB approval, we will be able to provide the required sample of Ohio State faculty and staff for your research. If you need any additional information, please feel free to contact me at 8-3347.

Sincerely,

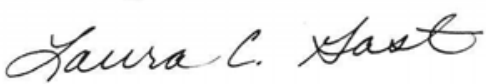

Laura C. Gast

HRITS Senior Research Consultant 
APPENDIX D

PARTICIPANT INVITATION AND FOLLOW-UP LETTERS 
Hello,

You are invited to be in a study about factors related to exercise in working adults. This is the dissertation research of Christopher Fink, who is working on his $\mathrm{PhD}$ under the supervision of Dr. Janet Buckworth at The Ohio State University.

We are trying to learn more about people who exercise and people who don't exercise. As long as you are between the ages of 18 and 65, you can be involved. It doesn't matter if you exercise or not - your participation in this study is very important.

If you volunteer, you will fill out an internet survey 3 times. You will complete the survey now, in 3 months, and in 6 months. You will spend no more than 15-20 minutes each time to complete the survey.

The information you provide for this study will be confidential, and none of your answers will be linked to you. Your personal answers will not be shared with your employer.

To fill out the survey, please enter the following web address into your browser:

http://education.osu.edu/paes/surveys/TakeSurvey.asp?SurveyID=3KM553K14535G

And enter the following username and password:

Username: websurvey

Password: survey

***Please note: If you need to return to the survey due to an error (computer lockup, power off), you may be asked for the Survey ID instead of the username and password. The survey ID is:

Survey ID: 3KM553K14535G

This invitation was also sent to your work email address, so you can simply copy and paste the link from there, as well. 
If you have questions or comments, please contact the researcher using the contact information listed below. This research project is sponsored by The Ohio State University School of Physical Activity and Educational Services. This research project has received approval from the Ohio State Institutional Review Board, protocol number $2006 \mathrm{E} 0090$.

Thank you in advance for taking the time to participate!

\begin{tabular}{|l|l}
\hline Christopher Fink & $\begin{array}{l}\text { Janet Buckworth, PhD, FACSM } \\
\text { Associate Professor } \\
\text { College of Education }\end{array}$ \\
Ph.D. Candidate & School of PAES \\
College of Education & The Ohio State University \\
School of PAES & 169 Cunz Hall \\
The Ohio State University & Columbus, OH 43210 \\
155 Cunz Hall & buckworth.1@osu.edu \\
Columbus, OH 43210 & \\
ph: (614) 570-6013 & \\
fink.93@osu.edu & \\
\hline
\end{tabular}


Hello,

About 10 days ago, you received a letter and email inviting you to be in a study about factors related to exercise in working adults. Your contribution to this research project is valued, and there is still time to participate! This is the dissertation research of Christopher Fink, who is working on his PhD under the supervision of Dr. Janet Buckworth at The Ohio State University.

If you are sure that you have already completed the entire survey, please ignore this reminder. We appreciate your participation! Also, if you receive an error while completing the survey, we urge you to try again later. It may just be overload from too many users at one time.

Again, we are trying to learn more about people who exercise and people who don't exercise. As long as you are between the ages of 18 and 65, you can be involved. It doesn't matter if you exercise or not - your participation in this study is very important.

If you volunteer, you will fill out an internet survey 3 times. You will complete the survey now, in 3 months, and in 6 months. You will spend no more than 15-20 minutes each time to complete the survey.

The information you provide for this study will be confidential, and none of your answers will be linked to you. Your personal answers will not be shared with your employer.

To fill out the survey, please enter the following web address into your browser:

http://education.osu.edu/paes/surveys/TakeSurvey.asp?SurveyID=3KM553K14535G

And enter the following username and password:

Username: websurvey

Password: survey

***Please note: If you need to return to the survey due to an error (computer lockup, power off), you may be asked for the Survey ID instead of the username and password. The survey ID is:

Survey ID: 3KM553K14535G

This invitation was also sent to your work mailing address, so you can simply copy and paste the link from there, as well. 
If you have questions or comments, please contact the researcher using the contact information listed below. This research project is sponsored by The Ohio State University School of Physical Activity and Educational Services. This research project has received approval from the Ohio State Institutional Review Board, protocol number 2006E0090.

Thank you in advance for taking the time to participate!

\begin{tabular}{|l|l|}
\hline Christopher Fink & Janet Buckworth, PhD, FACSM \\
& Associate Professor \\
Ph.D. Candidate & College of Education \\
College of Education & School of PAES \\
School of PAES & The Ohio State University \\
The Ohio State University & 169 Cunz Hall \\
155 Cunz Hall & Columbus, OH 43210 \\
Columbus, OH 43210 & buckworth.1@osu.edu \\
ph: (614) 570-6013 & \\
fink.93@osu.edu &
\end{tabular}


Hello,

Over the last month or so, you have received a few letters and email messages inviting you to be in a study about factors related to exercise in working adults. This is a final invitation to take part - we truly value your participation! This is the dissertation research of Christopher Fink, who is working on his PhD under the supervision of Dr. Janet Buckworth at The Ohio State University.

If you are sure that you have already completed the entire survey, please ignore this reminder. We appreciate your participation! Also, if you receive an error while completing the survey, we urge you to try again later. It may just be overload from too many users at one time.

Again, we are trying to learn more about people who exercise and people who don't exercise. As long as you are between the ages of 18 and 65, you can be involved. It doesn't matter if you exercise or not - your participation in this study is very important.

If you volunteer, you will fill out an internet survey 3 times. You will complete the survey now, in 3 months, and in 6 months. You will spend no more than 15-20 minutes each time to complete the survey.

The information you provide for this study will be confidential, and none of your answers will be linked to you. Your personal answers will not be shared with your employer.

To fill out the survey, please enter the following web address into your browser:

http://education.osu.edu/paes/surveys/TakeSurvey.asp?SurveyID=3KM553K14535G

And enter the following username and password:

Username: websurvey

Password: survey

***Please note: If you need to return to the survey due to an error (computer lockup, power off), you may be asked for the Survey ID instead of the username and password. The survey ID is:

Survey ID: 3KM553K14535G

This invitation was also sent to your work email address, so you can simply copy and paste the link from there, as well. 
If you have questions or comments, please contact the researcher using the contact information listed below. This research project is sponsored by The Ohio State University School of Physical Activity and Educational Services. This research project has received approval from the Ohio State Institutional Review Board, protocol number 2006E0090.

Thank you in advance for taking the time to participate!

\begin{tabular}{|l|l|}
\hline Christopher Fink & Janet Buckworth, PhD, FACSM \\
& Associate Professor \\
Ph.D. Candidate & College of Education \\
College of Education & School of PAES \\
School of PAES & The Ohio State University \\
The Ohio State University & 169 Cunz Hall \\
155 Cunz Hall & Columbus, OH 43210 \\
Columbus, OH 43210 & buckworth.1@osu.edu \\
ph: (614) 570-6013 & \\
fink.93@osu.edu &
\end{tabular}


Hi!

Thank you so much for returning to complete the final survey about factors related to exercise in working adults. Looking at the results, however, there seemed to be a problem with the submission. The data was not received properly, and therefore cannot be analyzed with the other study responses. Please try again to complete the survey at your earliest convenience. If you have any questions about the survey or its completion, please do not hesitate to email at fink.93@osu.edu. We truly appreciate you taking the time to assist us in this research.

To fill out the survey, please enter the following web address into your browser: http://education.osu.edu/paes/surveys/TakeSurvey.asp?SurveyID=3KM553K14535G

And enter the following username and password:

Username: websurvey

Password: survey

***Please note: If you need to return to the survey due to an error (computer lock-up, power off), you may be asked for the Survey ID instead of the username and password. The survey ID is:

\section{Survey ID: 3KM553K14535G}

This invitation was also sent to the email address you provided the first time, so you can simply copy and paste the link from there, as well.

If you have questions or comments, please contact the researcher using the contact information listed below. This research project is sponsored by The Ohio State University School of Physical Activity and Educational Services. This research project has received approval from the Ohio State Institutional Review Board, protocol number 2006E0090.

Thank you in advance for taking the time to participate!

\begin{tabular}{l|l}
\hline Christopher Fink & Janet Buckworth, PhD, FACSM \\
Ph.D. Candidate & Associate Professor \\
College of Education and Human Ecology & College of Education and Human Ecology \\
School of PAES & School of PAES \\
The Ohio State University & The Ohio State University \\
155 Cunz Hall & 169 Cunz Hall \\
Columbus, OH 43210 & Columbus, OH 43210 \\
ph: (614) 570-6013 & buckworth.1@,osu.edu \\
fink.93@osu.edu &
\end{tabular}


APPENDIX E

\section{WEB SURVEY - INTRODUCTION}


WEBS - Worksite Exercise Behavior Study

Welcome Back to WEBS!!!

We truly appreciate you returning to complete the 3 rd and final of 3 surveys!

As mentioned in the letter that you received, we are trying to learn more about people who exercise, and people who don't exercise. It doesn't matter if you exercise or not - your participation in this study is very important.

As a reminder, the information you provide for this study will be treated with absolute confidentiality. No responses will be individually identifiable or shared with your employer.

By completing this final survey, you are consenting to participation in research being conducted by Dr. Janet Buckworth and Christopher Fink of The Ohio State University and their assistants and associates.

The purpose of the study, the procedures that will be followed, and the amount of time the survey will take was explained in the invitation email and/or letter. You can choose not to participate without penalty to you. If you agree to participate, you can withdraw from the study at any time, and there will be no penalty.

After entering your email address below, you will be taken to the survey.

Your email address will ONLY be used to track those who have returned for the final survey. Your email address will not be connected in any way to your survey answers, and will not be shared with anyone.

1. Please enter the email address where you received your invitation to participate in this study below:*

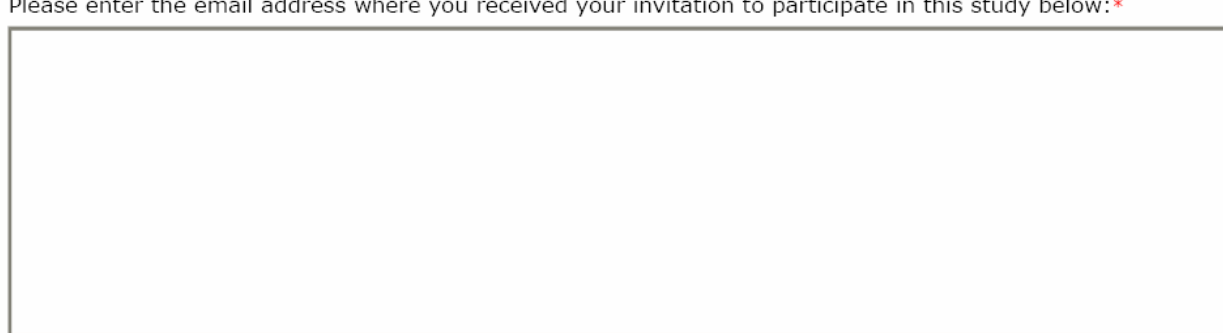

Once you have entered your email address, please click the 'NEXT' button below to begin the survey. 
Thank you for agreeing to participate in this study! The information you provide for this study will be treated with absolute confidentiality. No responses will be individually identifiable or shared with your employer.

Again, the purpose of the study, the procedures that will be followed, and the amount of time the survey will take was explained in the invitation email and/or letter. You can choose not to participate without penalty to you. If you agree to participate, you can withdraw from the study at any time, and there will be no penalty.

If you have questions, you can contact the investigators using the following information:

$\begin{array}{ll}\text { Christopher Fink } & \text { Janet Buckworth, PhD, } \\ \text { Ph.D. Candidate } & \text { FACSM } \\ \text { College of Education } & \text { Associate Professor } \\ \text { School of PAES } & \text { College of Education } \\ \text { The Ohio State } & \text { School of PAES } \\ \text { University } & \text { The Ohio State University } \\ \text { 340 Cunz Hall } & \text { 169 Cunz Hall } \\ \text { Columbus, OH 43210 } & \text { Columbus, OH 43210 } \\ \text { fink.93@osu.edu } & \text { buckworth.1@osu.edu }\end{array}$

For questions about your rights as a participant in this study or to discuss other study-related concerns or complaints with someone who is not part of the research team, please contact Ms. Sandra Meadows in The Ohio State University Office of Responsible Research Practices at 1-800-678-6251.

Please click 'NEXT' to start the survey.

Back
Next

Cancel 
APPENDIX F

WEB SURVEY - DEMOGRAPHICS 


\section{WEBS - Worksite Exercise Behavior Study}

Page 3 of 13

The following five questions will be used to match up the ID from the first survey, and will allow your responses to remain anonymous:

1. Please enter (below) the first letter of the company for which you work: For example, if you work for Ohio State University please enter ' $\mathrm{O}$ ' the space provided*

2. Please enter (below) the first letter of the city in which you were born:*

3. Please enter (below) the last digit of the zip code where you live: *

4. Please enter (below) the number of brothers that you have:*

5. Please enter (below) the third letter of the month you were born:*

6. What is your sex? (click the appropriate button)*

Male

Female

(2) Prefer not to answer

7. What is your age (in years)?*

The value must be between 18 and 65 , inclusive.

$$
\text { T }
$$

8. Are you Hispanic or Latino?*
Yes
No
Don't know/not sure
(2) Prefer not to answer 
9. Which one or more of the following would you say is your race? (click the boxes that apply)*

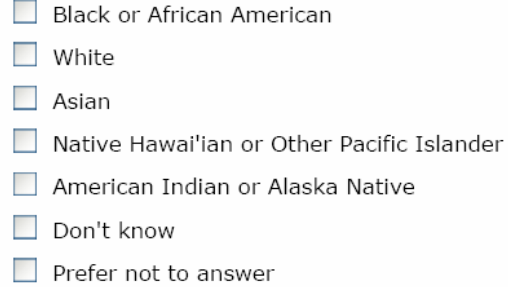

10. Are you...? (click the appropriate button)*

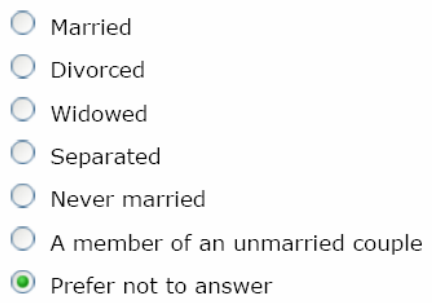

11. How tall are you (without shoes)?*

Feet

Inches

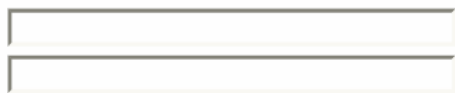

12. How much do you weigh (in pounds - without clothing)?*

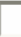

13. What is your highest level of education? (click the appropriate button)*

Did not finish high school

Graduated from high school

Attained a GED

Some education after high school / GED

Associate's Degree

Bachelor's Degree

Master's Degree

Professional Degree

Doctoral Degree

Post-Doctoral Training

Don't Know

(2) Prefer not to answer

14. What is your job status?*

Full-time

Part-time 

(2) Prefer not to answer

15. Please select the choice that most closely describes your job title:*
Executive
Senior level management
Professional / technical / research
Mid-level management
Clerical
Semi-skilled
C Prefer not to answer

Please review your answers, then click 'Next' to go to the next page. 
APPENDIX G

WEB SURVEY - CHECK FOR ILLNESS AND INJURY 
WEBS - Worksite Exercise Behavior Study

Page 4 of 13

Exercise includes activities such as brisk walking, jogging, swimming, aerobic dancing, biking, rowing, weight lifting, etc. Activities that are primarily sedentary such as bowling, or playing golf with a cart, would not be considered exercise.

1. Do you have any injuries or illnesses that will prevent you from exercising over the next 3 months? (click "YES" or "NO" below to answer) *

Yes $\mathrm{No}$

Please review your answer, then click 'Next' to go to the next page.

Back Next Cancel




\section{APPENDIX H \\ WEB SURVEY - STAGE OF CHANGE MEASURE \\ LONG / VIGOROUS / 5 CHOICE VERSION}


WEBS - Worksite Exercise Behavior Study

Page 5 of 13

Reminder:

Exercise includes activities such as brisk walking, jogging, swimming, aerobic dancing, biking, rowing, weight lifting, etc. Activities that are primarily sedentary such as bowling, or playing golf with a cart, would not be considered exercise.

1. Do you exercise regularly, that is, 3 or more times each week for at least 20 minutes each time? (Please click the button next to the one statement below that best describes you.*

Yes, and I have been for more than 6 months.

Yes, and I have been for less than 6 months.

No, but I am planning to start in the next 30 days.

No, but I am planning to start in the next 6 months.

No, and I don't have plans to start in the next 6 months.

Please review your answer, then click 'Next' to go to the next page. 
APPENDIX I

WEB SURVEY - EXERCISE RECALL - MODERATE 


\section{During the LAST 7 DAYS, how much TIME did you spend doing MODERATE} exercise?

MODERATE exercise is planned physical activity done to enhance health, which is continuous (10 minutes or more duration), mildly elevates heart rate mildly elevates breathing rate, allows conversation while exercising.

Examples include:

- weight lifting

- aerobic dance

- swimming (no laps)

- low-impact exercise/strength classes

- brisk walking

- light cycling, less than 3 miles

- recreational team sports (softball, volleyball, etc.)

- golfing without a cart

- hiking

- half-court basketball

- social dancing

- doubles tennis

1. Please click the button corresponding with the day of the week for YESTERDAY.*

$$
\text { Sunday Monday Tuesday Wednesday Thursday Friday Saturday }
$$

- 0

2. Did you engage in any moderate exercise YESTERDAY?*

3. For how many minutes did you participate in moderate-intensity activity YESTERDAY (if none, please enter ' 0 ')?

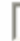

4. Please click in the box next to the activity(ies) in which you participated YESTERDAY.

$\square$ Brisk walking

$\square$ Light cycling (less than 3 miles)

$\square$ Low impact exercise/strength classes

$\square$ Swimming (no laps)

$\square$ Aerobic dance

$\square$ Recreational team sports

$\square$ Half-court basketball

$\square$ Hiking

$\square$ Golfing without a cart 
1 Social dancing

$\square$ Doubles tennis

$\square$ Weight lifting

$\square$ other, please specify

5. Please click the button corresponding with the day of the week for 2 DAYS AGO.*

$\begin{array}{ccccccc}\text { Sunday } & \text { Monday } & \text { Tuesday } & \text { Wednesday } & \text { Thursday } & \text { Friday } & \text { Saturday } \\ 0 & 0 & 0 & 0 & 0 & 0 & 0\end{array}$

6. Did you engage in any moderate exercise 2 DAYS AGO?*

Yes

No

Q

Q

7. For how many minutes did you participate in moderate-intensity activity 2 DAYS AGO (if none, please enter '0')?*

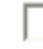

8. Please click in the box next to the activity(ies) in which you participated 2 DAYS AGO.

$\square$ Brisk walking

$\square$ Light cycling (less than 3 miles)

$\square$ Low impact exercise/strength classes

$\square$ Swimming (no laps)

$\square$ Aerobic dance

$\square$ Recreational team sports

$\square$ Half-court basketball

$\square$ Hiking

$\square$ Golfing without a cart

$\square$ Social dancing

$\square$ Doubles tennis

$\square$ Weight lifting

$\square$ Other, please specify

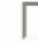

9. Please click the button corresponding with the day of the week for 3 DAYS AGO.*

$\begin{array}{ccccccc}\text { Sunday } & \text { Monday } & \text { Tuesday } & \text { Wednesday } & \text { Thursday } & \text { Friday } & \text { Saturday } \\ 0 & 0 & 0 & 0 & 0 & 0 & 0\end{array}$


10. Did you engage in any moderate exercise 3 DAYS AGO?*

11. For how many minutes did you participate in moderate-intensity activity 3 DAYS AGO (if none, please enter ' 0 ')?*

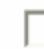

12. Please click in the box next to the activity(ies) in which you participated 3 DAYS AGO.
$\square$ Brisk walking
$\square$ Light cycling (less than 3 miles)
$\square$ Low impact exercise/strength classes
$\square$ Swimming (no laps)
$\square$ Aerobic dance
$\square$ Recreational team sports
$\square$ Half-court basketball
$\square$ Hiking
$\square$ Golfing without a cart
$\square$ Social dancing
$\square$ Doubles tennis
$\square$ Weight lifting
$\square$ Other, please specify

13. Please click the button corresponding with the day of the week for 4 DAYS AGO.*

$\begin{array}{ccccccc}\text { Sunday } & \text { Monday } & \text { Tuesday } & \text { Wednesday } & \text { Thursday } & \text { Friday } & \text { Saturday } \\ \text {. } & 0 & 0 & 0 & 0 & 0 & 0\end{array}$

14. Did you engage in any moderate exercise 4 DAYS AGO?*

15. For how many minutes did you participate in moderate-intensity activity 4 DAYS AGO (if none, please enter ' 0 ')?*

16. Please click in the box next to the activity(ies) in which you participated 4 DAYS AGO.
$\square$ Brisk walking
$\square$ Light cycling (less than 3 miles)
$\square$ Low impact exercise/strength classes
$\square$ Swimming (no laps)
$\square$ Aerobic dance 


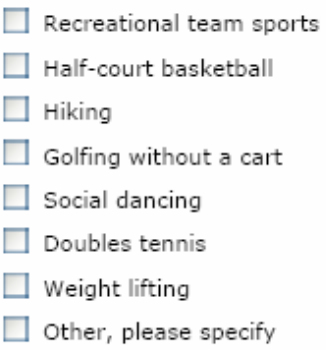

17. Please click the button corresponding with the day of the week for 5 DAYS AGO.*

$\begin{array}{ccccccc}\text { Sunday } & \text { Monday } & \text { Tuesday } & \text { Wednesday } & \text { Thursday } & \text { Friday } & \text { Saturday }\end{array}$

18. Did you engage in any moderate exercise 5 DAYS AGO?*

Yes No

O $\mathrm{O}$

19. For how many minutes did you participate in moderate-intensity activity 5 DAYS AGO (if none, please enter ' 0 ')?* $\Gamma$

20. Please click in the box next to the activity(ies) in which you participated 5 DAYS AGO.
$\square$ Brisk walking
$\square$ Light cycling (less than 3 miles)
$\square$ Low impact exercise/strength classes
$\square$ Swimming (no laps)
aerobic dance
$\square$ Recreational team sports
$\square$ Half-court basketbal!
$\square$ Hiking
$\square$ Golfing without a cart
$\square$ Social dancing
$\square$ Doubles tennis
$\square$ Weight lifting
$\square$ other, please specify 
21. Please click the button corresponding with the day of the week for 6 DAYS AGO.*

Sunday Monday Tuesday Wednesday Thursday Friday Saturday

. $\bigcirc \quad \bigcirc \quad 0 \quad 0 \quad 0 \quad 0$

22. Did you engage in any moderate exercise 6 DAYS AGO?*

Yes

No

- 0 O

23. For how many minutes did you participate in moderate-intensity activity 6 DAYS AGO (if none, please enter ' 0 ')?*

24. Please click in the box next to the activity(ies) in which you participated 6 DAYS AGO.
$\square$ Brisk walking
$\square$ Light cycling (less than 3 miles)
$\square$ Low impact exercise/strength classes
$\square$ Swimming (no laps)
$\square$ Aerobic dance
$\square$ Recreational team sports
$\square$ Half-court basketball
$\square$ Hiking
$\square$ Golfing without a cart
$\square$ Social dancing
$\square$ Doubles tennis
$\square$ Weight lifting
$\square$ other, please specify

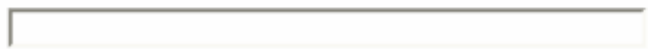

25. Please click the button corresponding with the day of the week for 7 DAYS AGO.*

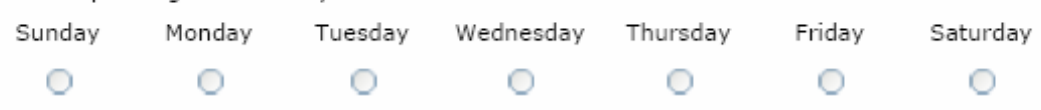

26. Did you engage in any moderate exercise 7 DAYS AGO?*

27. For how many minutes did you participate in moderate-intensity activity 7 DAYS AGO (if none, please enter '0')?* ( 
28. Please click in the box next to the activity(ies) in which you participated 7 DAYS AGO.

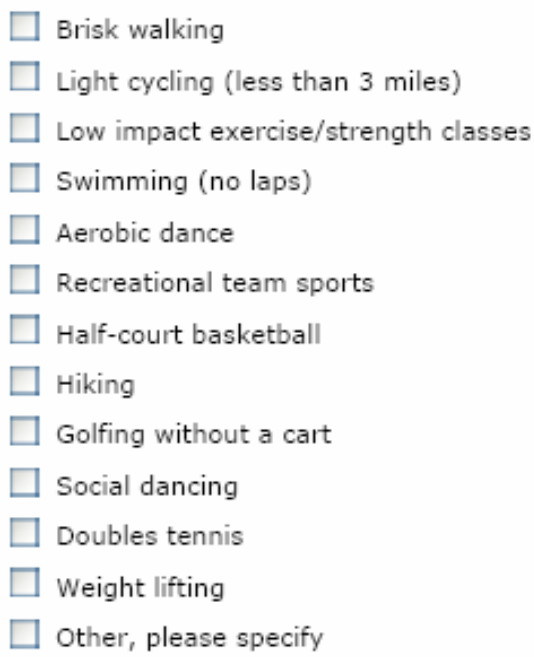

Please review your answers, then click 'Next' to go to the next page.

$$
\text { Back Next Cancel }
$$


APPENDIX J

WEB SURVEY - EXERCISE RECALL - VIGOROUS 
WEBS - Worksite Exercise Behavior Study

Page 7 of 13

During the LAST 7 DAYS, how much TIME did you spend doing VIGOROUS exercise?

VIGOROUS exercise is planned physical activity done to enhance health, which is continuous ( 20 minutes or more duration), causes the heart to beat rapidly, causes breathing to be rapid and deep, and does not allow conversation while exercising.

Examples include:

- running or jogging

- high-intensity aerobics classes

- competitive field sports (i.e. soccer)

- competitive full-court basketball

- cycling (10mph for more than 3 miles)

- swimming laps

- martial arts

- calisthenics (push-ups, sit-ups)

1. Please click the button corresponding with the day of the week for YESTERDAY.*

Sunday Monday Tuesday Wednesday Thursday Friday Saturday

2. Did you engage in any vigorous exercise YESTERDAY?*

Yes No

3. For how many minutes did you participate in vigorous-intensity activity YESTERDAY (if none, please enter 'O')?*

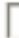

4. Please click in the box next to the activity(ies) in which you participated YESTERDAY.

$\square$ Running or jogging

$\square$ High-intensity aerobics classes

$\square$ Competitive field sports (soccer)

$\square$ Competitive full-court basketbal

$\square$ Cycling ( $10 \mathrm{mph}$ for more than 3 miles)

$\square$ Swimming laps

$\square$ Martial arts

$\square$ Calisthenics (push-ups, sit-ups)

$\square$ Other, please specify 
5. Please click the button corresponding with the day of the week for 2 DAYS AGO.

$\begin{array}{ccccccc}\text { Sunday } & \text { Monday } & \text { Tuesday } & \text { Wednesday } & \text { Thursday } & \text { Friday } & \text { Saturday } \\ 0 & 0 & 0 & 0 & 0 & 0 & 0\end{array}$

6. Did you engage in any vigorous exercise 2 DAYS AGO?*

Yes

No

Q

Q

7. For how many minutes did you participate in vigorous-intensity activity 2 DAYS AGO (if none, please enter ' 0 ')?"

8. Please click in the box next to the activity(ies) in which you participated 2 DAYS AGO.

$\square$ Running or jogging

$\square$ High-intensity aerobics classes

$\square$ Competitive field sports (soccer)

$\square$ Competitive full-court basketbal

$\square$ Cycling (10mph for more than 3 miles)

$\square$ Swimming laps

$\square$ Martial arts

$\square$ Calisthenics (push-ups, sit-ups)

$\square$ Other, please specify

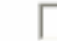

9. Please click the button corresponding with the day of the week for 3 DAYS AGO.

$\begin{array}{ccccccc}\text { Sunday } & \text { Monday } & \text { Tuesday } & \text { Wednesday } & \text { Thursday } & \text { Friday } & \text { Saturday } \\ \bigcirc & \bigcirc & \bigcirc & \bigcirc & \bigcirc & \bigcirc & \bigcirc\end{array}$

10. Did you engage in any vigorous exercise 3 DAYS AGO?*

11. For how many minutes did you participate in vigorous-intensity activity 3 DAYS AGO (if none, please enter ' 0 ')?*

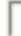

12. Please click in the box next to the activity(ies) in which you participated 3 DAYS AGO.

$\square$ Running or jogging 


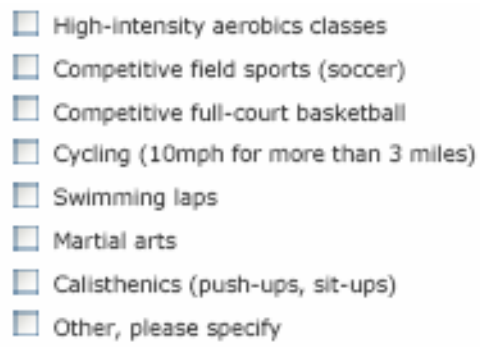

$\square$ Other, please specify

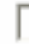

13. Please click the button corresponding with the day of the week for 4 DAYS AGO.*

$\begin{array}{ccccccc}\text { Sunday } & \text { Monday } & \text { Tuesday } & \text { Wednesday } & \text { Thursday } & \text { Friday } & \text { Saturday } \\ 0 & 0 & 0 & 0 & 0 & 0 & 0\end{array}$

14. Did you engage in any vigorous exercise 4 DAYS AGO?*

Yes No

15. For how many minutes did you participate in vigorous-intensity activity 4 DAYS AGO (if none, please enter ' 0 ')?*

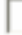

16. Please click in the box next to the activity(ies) in which you participated 4 DAYS AGO.
$\square$ Running or jogging
$\square$ High-intensity aerobics classes
$\square$ Competitive field sports (soccer)
$\square$ Competitive full-court basketball
$\square$ cycling (10mph for more than 3 miles)
$\square$ Swimming laps
$\square$ Martial arts
$\square$ Calisthenics (push-ups, sit-ups)
$\square$ Other, please specify

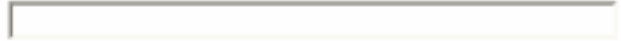

17. Please click the button corresponding with the day of the week for 5 DAYS AGO.*

$\begin{array}{ccccccc}\text { Sunday } & \text { Monday } & \text { Tuesday } & \text { Wednesday } & \text { Thursday } & \text { Friday } & \text { Saturday } \\ \bigcirc & \bigcirc & \bigcirc & \bigcirc & \bigcirc & \bigcirc & \emptyset\end{array}$


18. Did you engage in any vigorous exercise 5 DAYS AGO?*

19. For how many minutes did you participate in vigorous-intensity activity 5 DAYS AGO (if none, please enter '0')?*

20. Please click in the box next to the activity(ies) in which you participated 5 DAYS AGO.
$\square$ Running or jogging
$\square$ High-intensity aerobics classes
$\square$ Competitive field sports (soccer)
$\square$ Competitive full-court basketball
$\square$ Cycling (10mph for more than 3 miles)
$\square$ Swimming laps
$\square$ Martial arts
$\square$ Calisthenics (push-ups, sit-ups)
$\square$ Other, please specify

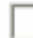

21. Please click the button corresponding with the day of the week for 6 DAYS AGO.*

$\begin{array}{ccccccc}\text { Sunday } & \text { Monday } & \text { Tuesday } & \text { Wednesday } & \text { Thursday } & \text { Friday } & \text { Saturday } \\ 0 & 0 & 0 & 0 & 0 & 0 & 0\end{array}$

22. Did you engage in any vigorous exercise 6 DAYS AGO?*

O

23. For how many minutes did you participate in vigorous-intensity activity 6 DAYS AGO (if none, please enter ' 0 ')?*

24. Please click in the box next to the activity(ies) in which you participated 6 DAYS AGO.
$\square$ Running or jogging
$\square$ High-intensity aerobics classes
$\square$ Competitive field sports (soccer)
$\square$ Competitive full-court basketball
$\square$ Cycling ( $10 \mathrm{mph}$ for more than 3 miles)
$\square$ Swimming laps
$\square$ Martial arts
$\square$ Calisthenics (push-ups, sit-ups)
$\square$ Other, please specify 
25. Please click the button corresponding with the day of the week for 7 DAYS AGO.*

$\begin{array}{cccccccc}\text { Sunday } & \text { Monday } & \text { Tuesday } & \text { Wednesday } & \text { Thursday Friday } & \text { Saturday }\end{array}$

26. Did you engage in any vigorous exercise 7 DAYS AGO?*

27. For how many minutes did you participate in vigorous-intensity activity 7 DAYS AGO (if none, please enter 'O')?" T

28. Please click in the box next to the activity(ies) in which you participated 7 DAYS AGO.
$\square$ Running or jogging
$\square$ High-intensity aerobics classes
$\square$ Competitive field sports (soccer)
$\square$ Competitive full-court basketbal
$\square$ Cycling (10mph for more than 3 miles)
$\square$ Swimming laps
$\square$ Martial arts
$\square$ Calisthenics (push-ups, sit-ups)
$\square$ Other, please specify<smiles>C1CCC1</smiles>

Please review your answers, then click 'Next' to go to the next page.

Back Next Cancel




\section{APPENDIX K \\ WEB SURVEY - PROCESSES OF CHANGE}


WEBS - Worksite Exercise Behavior Study

Page 11 of 13

The following experiences can affect the exercise habits of some people. Think of any similar experiences you may be currently having or have had during the past month. Then, rate how frequently the event occurs. Using the rating system shown below, please click the button below the number that best describes your answer.

How frequently does this occur?

$1=$ Never

$3=$ Occasionally

$5=$ Repeatedly

1. Instead of remaining inactive, I engage in some physical activity.*

$1=$ Never, $3=$ Occasionally, $5=$ Repeatedly

$\begin{array}{ccccc}1 & 2 & 3 & 4 & 5 \\ 0 & 0 & 0 & 0 & 0\end{array}$

2. I tell myself I am able to keep exercising if I want to.*

$1=$ Never, $3=$ Occasionally, $5=$ Repeatedly

1

23

4

5

(

3. I keep a set of exercise clothes conveniently located so that I can exercise whenever I get the time. * $1=$ Never, $3=$ Occasionally, $5=$ Repeatedly

2

\section{3}

4

5

○

4. I tell myself that I can keep exercising if I try hard enough.* $1=$ Never, $3=$ Occasionally, $5=$ Repeatedly

\section{1}

○

\section{2}

○

5. I find out about new methods of exercising.* $1=$ Never, $3=$ Occasionally, $5=$ Repeatedly

6. I make commitments to exercise.*

$1=$ Never, $3=$ Occasionally, $5=$ Repeatedly

\section{1} $\bigcirc$ $\bigcirc$

\section{3}

4

5

O

3

0

O

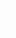


7. One of the rewards of regular exercise is that it improves my mood." $1=$ Never, 3=Occasionally, S=Repeatedly

$\begin{array}{lllll}1 & 3 & 4 & 5 & 5 \\ 0 & 0 & 0 & 0 & 0\end{array}$

8. I think about information from articles and advertisements on how to make exercise a regular part of my life.* $1=$ Never, 3=Occasionally, $\mathrm{S}=$ flepeatedly

$\begin{array}{lllll}1 & 2 & 3 & 4 & 5 \\ 0 & 0 & 0 & 0 & 0\end{array}$

9. I keep things around my place of work that remind me to exercise.* $1=$ Never, 3-Occasionally, S=flepeatedly

$\begin{array}{lllll}1 & 2 & 3 & 4 & 5 \\ 0 & 0 & 0 & \bigcirc & 0\end{array}$

10. I find society changing in ways that make it easier to exercise.* $1=$ Never, 3=0ccasionally, $S=$ Repeatedly

$\begin{array}{lllll}1 & 2 & 3 & 4 & 5 \\ 0 & \bigcirc & \bigcirc & \bigcirc & \bigcirc\end{array}$

11. I am afraid of the consequences to my health if $\mathrm{t}$ do not exercise.* $1=$ Never, 3=Occasionally, $S=$ flepeatedly

$\begin{array}{lllll}1 & 2 & 3 & 4 & 5 \\ 0 & 0 & 0 & 0 & 0\end{array}$

12. I get upset when I realize that people I love would have better health if they exercised. * $1=$ Never, 3=Occasionally, $5=$ feepeatedly

$\begin{array}{lllll}1 & 2 & 3 & 4 & 5 \\ 0 & 0 & 0 & 0 & 0\end{array}$

13. I react emotionally to the warnings about an inactive lifestyle.* $1=$ Never, 3=0ccasionally, $5=$ Repeatedly

$\begin{array}{lllll}1 & 3 & 4 & 5 \\ 0 & \bigcirc & 0 & 0 & \bigcirc\end{array}$

14. I get upset when I see people who would benefit from exercise but choose not to exercise." $1=$ Never, 3=Occasionally, $5=$ Repeatedly,

$\begin{array}{lllll}1 & 2 & 3 & 4 & 5 \\ 0 & 0 & 0 & 0 & 0\end{array}$

15. I believe that regular exercise would make me a healthier, happler person. * $1=$ Never, 3=Occasionally, $5=$ Fiepeatedly

$\begin{array}{lllll}1 & 2 & 3 & 4 & 5 \\ 0 & 0 & 0 & 0 & 0\end{array}$


16. I have a workout partner.*

$1=$ Never, $3=$ Occasionally, S=flepeatedly

$\begin{array}{lllll}1 & 3 & 4 & 5\end{array}$

17. I seek information about which exercises are best for me.* $1=$ Never, 3=Occasionally, $5=$ Flepeatedly

0

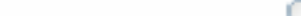

(O)

(9)

18. I congratulate myself after I exercise.*

$1=$ Never, $3=0$ occasionally, $5=$ flepeatedly

$\begin{array}{lllll}1 & 2 & 3 & 4 & 5 \\ 0 & 0 & 0 & 0 & 0\end{array}$

19. I have a friend who encourages me to exercise when I don't feel up to it." $1=$ Never, $3=0$ ccasionally, $5=$ flepeatedly.

$\begin{array}{lllll}1 & 2 & 3 & 4 & 5 \\ 0 & 0 & 0 & \bigcirc & 0\end{array}$

20. If $I$ engage in regular exercise, $I$ find that $I$ get the benefit of having more energy. $1=$ Never, $3=0$ ccasionally, $5=$ Repeatedly

$\begin{array}{lllll}1 & 2 & 3 & 4 & 5 \\ 0 & 0 & 0 & 0 & 0\end{array}$

Please review your answers, then click 'Next' to go to the next page. 
WEBS - Worksite Exercise Behavior Study

Page 12 of 13

The following experiences can affect the exercise habits of some people. Think of any similar experiences you may be currently having or have had during the past month. Then, rate how frequently the event occurs. Using the rating system shown below, please click the button below the number that best describes your answer.

How frequently does this ocour?

$1=$ Never

$3=$ Occasionally

$5=$ Repeatedly

1. Rather than viewing exercise as another task to get out of the way, 1 try to use it as my time to relax. $1=$ Never, $3=$ Occasionally, $5=$ flepeatedly

$\begin{array}{lllll}1 & 2 & 3 & 4 & 5 \\ 0 & 0 & 0 & 0 & 0\end{array}$

2. I am aware that communities are spending more money on recreation centers and bike paths. $1=$ Never, 3=0ccasionally, $5=$ flepeatedly

$\begin{array}{lllll}1 & 2 & 3 & 4 & 5 \\ 0 & 0 & 0 & 0 & 0\end{array}$

3. I do something nice for myself for making efforts to exercise more." $1=$ Never, $3=$ Occasionaly, $5=$ Repeated ly

$\begin{array}{lllll}1 & 2 & 3 & 4 & 5 \\ 0 & 0 & 0 & \bigcirc & 0\end{array}$

4. I have someone who encourages me to exercise.* $1=$ Never, $3=$ Occasionally, $5=$ flepeatedly

$\begin{array}{lllll}1 & 2 & 3 & 4 & 5 \\ 0 & \bigcirc & 0 & \bigcirc & 0\end{array}$

5. I have someone who provides feedback about my exercising." $1=$ Never, $3=$ Occasionally, $5=$ flepeatedly

12

6. I bring my athletic shoes to work so I will remember to take a walk at lunch time.* $1=$ Never, $3=$ Occasionally, $5=$ flepeatedly

$\begin{array}{lllll}1 & 2 & 3 & 4 & 5 \\ 0 & 0 & 0 & 0 & 0\end{array}$


7. I believe that 1 can exercise regularly.*

1 =Never, 3=Occasionally, $5=$ =fepeatedly

$\begin{array}{lllll}1 & 2 & 3 & 4 & 5 \\ 0 & 9 & 0 & 9 & 9\end{array}$

8. I look for information related to exercise.

1 =Never, 3=Occasionally, S=flepeatedly

$\begin{array}{lllll}1 & 2 & 3 & 4 & 5 \\ 0 & 0 & 0 & 0 & 0\end{array}$

9. I use my calendar to schedule my exercise time.*

1 =Never, 3=Occasionaly, $5=$ flepeatedly

$\begin{array}{lllll}2 & 2 & 3 & 4 & 5 \\ 0 & 0 & 0 & \bigcirc & 0\end{array}$

10. I feel I would be a better role model for others if I exercised regularly.* $1=$ Never, $3=$ Occasionally, $5=$ flepeatedly

$\begin{array}{lllll}2 & 3 & 4 & 5 \\ 0 & \bigcirc & \bigcirc & \bigcirc & \bigcirc\end{array}$

11. I feel more confident when I exercise regularly.*

1 =Never, 3=Occasionally, S=Repeatedly

$\begin{array}{lllll}1 & 2 & 3 & 4 & 5 \\ 0 & 0 & 0 & 0 & 0\end{array}$

12. I notice that more business are encouraging their employees to exercise by offering fitness courses and time off work out.

$1=$ Never, 3=Occasionally, $S=$ flepeatedly

$\begin{array}{lllll}2 & 3 & 4 & 5 \\ 0 & 0 & 0 & 0 & 0\end{array}$

13. I wonder how my inactivity affects those people who are close to me.*

$1=$ Never, 3=Occasionally, S=Repeatedly

$\begin{array}{lllll}1 & 2 & 3 & 4 & 5 \\ 0 & 0 & 0 & 0 & 0\end{array}$

14. I realize that I might be able to encourage others to be healthier if I would exercise more. $1=$ Never, 3=Occasionally, $5=$ =flepeatedly

$\begin{array}{lllll}1 & 2 & 3 & 4 & 5 \\ 0 & 0 & 0 & 0 & 0\end{array}$

15. I get frustrated with myself when I don't exercise.* $1=$ Never, 3=Occasionally, $5=$ flepeatedly

$\begin{array}{lllll}1 & 2 & 3 & 4 & 5 \\ 0 & \bigcirc & 0 & 0 & 0\end{array}$


16. I have noticed that famous people often advertise the fact that they exercise regularly. $1=$ Never, $3=$ Occasionally, $5=$ flepeatedly

$\begin{array}{lllll}2 & 3 & 4 & 5 \\ 0 & 0 & 0 & \bigcirc & \bigcirc\end{array}$

17. Some of my closest friends might exercise more if I would.* $1=$ Never, $3=$ Occasionally, $5=$ Repeatedly

$\begin{array}{lllll}1 & 2 & 3 & 4 & 5 \\ 0 & 0 & 0 & 0 & 0\end{array}$

18. I think that because I value health $\mathrm{I}$ should value exercise.* i=Never, 3=Occasionally, 5=Repeatedly

$\begin{array}{lllll}1 & 2 & 3 & 4 & 5 \\ 0 & 0 & 0 & 0 & 0\end{array}$

19. Instead of relaxing by watching TV or eating, 1 take a walk or exercise.* $1=$ Never, 3=Occasionally, $5=$ Repeatedly

$\begin{array}{lllll}2 & 3 & 4 & 5 \\ 0 & 0 & 0 & 0 & 0\end{array}$

20. I think of exercise as fun rather than a burden.* $1=$ Never, 3=Occasionally, $5=$ Fiepeatedly

$\begin{array}{lllll}1 & 2 & 3 & 4 & 5 \\ 0 & 0 & 0 & 0 & 0\end{array}$

Please review your answers, then click 'Next' to go to the next page. 
APPENDIX L

WEB SURVEY - DECISIONAL BALANCE 
Please rate how important each of these statements is in your decision whether or not to exercise. In each case, think about how you feel right now not how you have felt in the past or would like to feel. Using the rating system shown below, please click the circle below each statement that best describes your answer.

The rating scale is as follows:

$1=$ Not at all important (in my decision whether or not to exercise). $3=$ Moderately important (in my decision whether or not to exercise). $5=$ Extremely important (in my decision whether or not to exercise).

1. I would have more energy for my family and friends if I exercised regularly.* 1 =Not at all important, 3=Moderately important, 5=Extremely important

$\begin{array}{lllll}1 & 2 & 3 & 4 & 5 \\ 0 & 0 & 0 & 0 & 0\end{array}$

2. Regular exercise would help me relieve tension.* $1=$ Not at all important, 3=Moderately important, $5=$ Extremely important

$\begin{array}{lll}1 & 2 & 3 \\ 0 & 0 & 0\end{array}$

.

0

O

3. I think I would be too tired to do my daily work after exercising.*

$\begin{array}{cccccc}1 & 2 & 3 & 4 & 5 \\ 0 & 0 & 0 & 0 & 0\end{array}$

4. I would feel more confident if $\mathrm{I}$ exercised regularly.

$1=$ Not at all important, 3=Moderately important, 5= Extremely important

$\begin{array}{lllll}1 & 2 & 3 & 4 & 5 \\ 0 & 0 & 0 & 0 & 0\end{array}$

5. I would sleep more soundly if I exercised regularly.

$1=$ Not at all important, 3=Moderately important, 5= Extremely important

2

4

0

6. I would feel good about myself if I kept my commitment to exercise regularly.* 1 =Not at all important, 3=Moderately important, 5=Extremely important 
7. I would find it difficult to find an exerose activity that $I$ enjoy that is not affected by bad weather.* $1=$ Not at all important, 3=Moderately important, 5=Extremely important

$\begin{array}{lllll}1 & 2 & 3 & 4 & 5 \\ 0 & 0 & 0 & \bigcirc & 0\end{array}$

8. I would like my body better if I exercised regularly.

$1=$ Not at all important, 3=Moderately important, 5=Extremely important

$\begin{array}{llccc}1 & 2 & 3 & 4 & 5 \\ 0 & 0 & 0 & 0 & 0\end{array}$

9. It would be easier for me to perform routine physical tasks if I exercised regularly.* $1=$ Not at all important, 3=Moderately important, 5=Extremely important

$\begin{array}{lllll}1 & 2 & 3 & 4 & 5 \\ 0 & 0 & 0 & 0 & 0\end{array}$

10. I would feel less stressed if $\mathrm{I}$ exercised regularly.*

$1=$ Not at all important, 3=Moderately important, $5=$ Extremely important

$\begin{array}{lllll}2 & 3 & 4 & 5 \\ 0 & \bigcirc & \bigcirc & \bigcirc & \bigcirc\end{array}$

11. I feel uncomfortable when I exercise because I get out of breath and my heart beats very fast. * $1=$ Not at all important, 3=Moderately important, 5=Extremely important

$\begin{array}{lllll}2 & 3 & 4 & 5\end{array}$

Q

Q

12. I would feel more comfortable with my body if $\mathrm{I}$ exercised regularly.* $1=$ Not at all important, 3=Moderately important, 5=Extremely important

$\begin{array}{lllll}1 & 2 & 3 & 4 & 5 \\ 0 & 0 & 0 & 0 & 0\end{array}$

13. Regular exercise would take too much of my time.*

$1=$ Not at all important, 3=Moderately important, 5=Extremely important

$\begin{array}{lllll}1 & 2 & 3 & 4 & 5 \\ 0 & 0 & 0 & 0 & 0\end{array}$

14. Regular exercise would help me have a more positive outlook on life.* $1=$ Not at all important, 3=Moderately important, 5=Extremely important

$\begin{array}{llccc}1 & 2 & 3 & 4 & 5 \\ 0 & 0 & 0 & 0 & 0\end{array}$

15. I would have less time with my family and friends if $\mathrm{I}$ exercised regularly. $1=$ Not at all important, 3=Moderately important, 5=Extremely important

$\begin{array}{lllll}1 & 2 & 3 & 4 & 5 \\ 0 & 0 & 0 & 0 & 0\end{array}$


16. At the end of the day, I am too exhausted to exerose.

$1=$ Not at all important, 3=Moderately important, 5= Extremely important

$\begin{array}{llccc}2 & 3 & 4 & 5 \\ 0 & \bigcirc & \bigcirc & \bigcirc & \bigcirc\end{array}$

Please review your answers, then click 'Next' to go to the next page.

Back Next Cancel 
APPENDIX M

WEB SURVEY - SELF-EFFICACY 


\section{WEBS - Worksite Exercise Behavior Study}

Page 9 of 13

Please click the appropriate button to rate how confident you are that you could exercise in each of the following situations.

Rating Scale:

$1=$ Not at all confident

3=Moderately confident

5=Extremely confident

1. When I am tired.

$1=$ Not at all confident, 3=Moderately confident, 5=Extremely confident

1

2

2. When I am in a bad mood.*

$1=$ Not at all confident, $3=$ Moderately confident, 5=Extremely confident

12

3. When I don't have the time.*

$1=$ Not at all confident, $3=$ Moderately confident, 5=Extremely confident

12

4. When I am on vacation.*

$1=$ Not at all confident, $3=$ Moderately confident, $5=$ Extremely confident

12

23

3

4

4

5

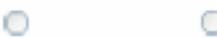

9

5. When it is raining or snowing.*

$1=$ Not at all confident, $3=$ Moderately confident, $5=$ Extremely confident

$\begin{array}{lcccc}1 & 2 & 3 & 4 & 5 \\ 0 & 0 & 0 & 0 & 0\end{array}$

Please review your answers, then click 'Next' to go to the next page.

Back Next Cancel


APPENDIX N

WEB SURVEY - INTENTION 
WEBS - Worksite Exercise Behavior Study

Page 8 of 13

Remember!! Exercise includes activities such as brisk walking, jogging, swimming, aerobic dancing, biking, rowing, weight lifting, etc. Activities that are primarily sedentary such as bowling, or playing golf with a cart, would not be considered exercise.

Please click the appropriate button to rate how true you feel the following statements are for you:

1. I intend to exercise for 20 minutes or longer, at least on three days per week and on a regular basis.*

$$
\text { Not at all true } \quad \text { Not true } \quad \text { A little true } \quad \text { Absolutely true }
$$

2. I intend to exercise occasionally, for 20 minutes or longer, on at least three days per week (at least once a month).*
Not at all true
Not true
A little true
Absolutely true

3. I intend to perform fitness and muscle strengthening activities.*
Not at all true
Not true
A little true
Absolutely true

O

○

Please review your answers, then click 'Next' to go to the next page. 


\section{APPENDIX O}

WEB SURVEY - STAGE OF CHANGE

LONG / VIGOROUS / TRUE-FALSE VERSION 


\section{WEBS - Worksite Exercise Behavior Study}

Please use the following definition of exercise when answering these questions:

Exercise includes activities such as brisk walking, jogging, swimming, aerobic dancing, biking, rowing, weight lifting, etc. Activities that are primarily sedentary such as bowling, or playing golf with a cart, would not be considered exercise.

Please answer YES or NO to the next five statements by clicking on the choice BELOW the statement:

Remember: Regular exercise is performed 3 or more times each week for at least 20 minutes each time.

1. I currently exercise.*

Yes No

2. I intend to exercise in the next 6 months.*

3. I currently exercise regularly.*

4. I have exercised regularly for the past 6 months.*

Yes

5. I have exercised regularly in the past for at least 3 months.*

Please review your answers, then click 'Done' to go to the final page. 


\section{APPENDIX P}

WEB SURVEY - THANK YOU PAGE 


\section{WEBS - Worksite Exercise Behavior Study}

\section{Survey Completed}

Thank you! Your answers have been submitted, and will be kept confidential. You have completed the 3rd and final survey of this research study. Thank you again for taking the time to participate. Your input is valuable in helping us understand exercise among working adults! 